\title{
XXIX. Ueber Anglesit von Monteponi (Sardinien).
}

\author{
Von \\ P. Hermann in Mannheim.
}

(Hierzu Taf. XI-XIII.)

Ueber den Anglesit besitzen wir eine reiche Literatur, denn dieses Mineral hat stets durch seine Schönheit, durch die Schärfe seiner Ausbildung und durch den Reichthum an Formen und Typen das Interesse der Mineralogen auf sich gelenkt. Es bildet sich in der Regel durch Oxydation aus Bleiglanz, und es sind daher in den oberen Teufen der Bleierzgruben, gegen den Ausstrich der Erzgänge zu, die Bedingungen zu seiner Bildung gegeben. Wir kennen daher dieses Mineral von sehr vielen Fundorten, die über den ganzen Erdball verstreut sind.

Der bekannteste, man kann wohl sagen, der berühmteste Fundort für Anglesit ist die Grube Monteponi bei Iglesias in Sardinien. Anglesite von Monteponi finden sich in allen Sammlungen. Dem dortigen Vorkommen kommt der Umstand zu gute, dass der ausgezeichnete Leiter der Gruben, Herr Director E. Ferraris, aus Liebe zur Mineralogie dafür sorgt, dass die interessanten und werthvollen Mineralien seiner Bergwerke gerettet und der Wissenschaft zugeführt werden.

Durch seine Güte kam eine reiche und prächtige Sammlung von Anglesiten von Monteponi in den Besitz des Herrn Prof. Dr. V. Goldschmidt in Heidelberg, der mir dieselben zur Untersuchung anvertraute.

Wie oben gesagt wurde, besitzen wir über dieses Mineral ausführliche Publicationen hervorragender Krystallographen. Die wichtigste ist die Monographie von V. v. Lang (Wien. Sitzber. 1859, 36, 241), in der er die Angaben seiner Vorgänger zusammenfasst und unsere Kenntnisse durch eigene Beobachtungen an dem reichen Materiale der Wiener Sammlungen wesentlich erweitert.

In Lang's Monographie findet sich ein ausführliches Literaturverzeichniss, das bis zum Jahre 1859 reicht. Da aber seit dieser Zeit viele Untersuchungen über Anglesit publicirt worden sind, so mag es von Werth 
sein, eine Ergänzung dieses Verzeichnisses zu besitzen. Eine solche Ergänzung, die sich vorzugsweise auf Beschreibungen der Krystallformen bezieht, möge im Folgenden gegeben werden. Darin sind die Literaturangaben von Lang mit aufgenommen.

M. Monnet, Syst. min. 1779, 371.

\section{Literatur.}

R. Kirwa n, Elem. min. 1784, 301.

C. A. Gerhardt, Grundr. des Min.-Syst. 1876, 244.

v. Trebra, Crell's chem. Ann. 1786, 2, 328.

Proust, Obs. s. 1. phys. etc. p. Rozière et p. de la Methérie 1787, 30, 373.

G. S. O. Lasius, Beob. über die Harzgeb. 1789, 2, 355 .

J. J. Bind heim, Beob. etc. Ges. naturf. Freunde, Berlin 1792, 4, 367.

J. G. Schmeisser, Syst. min. 1795, 2, 181.

J. G. de Lamethérie, Théorie de la terre 1797, 1.

L. J. Jordan, Min. und chem. Beob. und Erfahr. 1800, 257.

Klap roth, Beitr. z. chem. Kennt. d. Min. 1802, 3, 162.

Hausmann, Norddeutsche Beitr. z. Berg- u. Hüttenk. 1807, 2, 11.

F. Strohmeyer, $\left\{\begin{array}{l}\text { Göttínger gelehrte Anzeigen 1812, } 204 . \\ \text { Ann. d. Phys. von Gilbert 1813, 44, } 209 .\end{array}\right.$

L. J. Jordan, Ann. d. Phys. von Gilbert 1813, 44, 213.

M. Meade, Amer. min. Journ. by A. Bruce 1814, 1, 150.

S. Leman, Bulletin de la soc. philomatique 1815, 65.

D) unin-Bork owsky, Leonh. Taschenb. f. gesaminte Min, 1816, 10, 296.

C. $\Lambda$. Zipser, Versuch eines topogr. min. Handb. v. Ungarn 1817, 431.

A. G. Werner, Letztes Min.-Syst. 1817, 262.

Selb, Leonl. Taschenb. f. d. ges. Min. 1818, 12, 312.

C. S. A. Il off man n, Handb. d. Min., fortges. v. Bre ithaupt 1818, 4 (II), 93 .

Bl u m h of, Leonh. Taschenb. f. d. ges. Min. $1821,15,312$.

B. Silliman, Amer. Journ. of Science and Arts 1821, 3, 173.

C. C. v. Le on har d, Handb. d. Orykt. 1. Aufl. 1821, 232.

Ha üy, Traité de min. éd. 1, 1801, 8, 503.

$$
\text { - - - - - 2, 1822, 3, } 403 \text {. }
$$

P. Cleaveland, Elem. Treatise on Min. and Geol. ed. 2, 1822, 2, 518, 721.

W. Phillips, Introd. to the Knowl. of Min. ed. 1, 1818, 217; ed. 3, 1823.

c. Hartmann, Leonh. Taschenb. f. d. ges. Min. 1824, 15, 946.

A. T. Kupfer, Preisschr. Berlin 1825.

F. Mohs, Grundr. d. Min. 1824, 2, 163.

Mohs-Haidinger, Treat. on Min. 1825, 2, 142.

C. F. Naumann, Isis von Oken 1826, 688 (Dover).

k. F. A. Hartman n, Handwörterb. d. Min. u. Geogn. 1828, 72.

C. F. Na umann, Lehrb. d. Min. 1828, 324.

F. A. Walchner, Handb. d. ges. Min. 1829, Abth. I, 478.

F. S. Beudant, Traité d. Min. éd. 2, 1832, $2,459$.

C. C. v. Leonhard, Grundr. d. Orykt. 1833, 126.

J. F. W. Johnston, Report of th Brit. Assoc. 1833, 572.

R. $\Lambda l l a$ a , Man. of Min. 1834, 56.

E. Kayser, Beschr. d. Samml, d. H. Bergmann $1834,359$.

Ch. U. Shepard, Treat. on Min. Abth. II, 183\%, 1, 19.

G. Rose, Min. geogn. Reise n. d. Ural etc. 1837, 1, 211. 
A. Lé vy, Description etc. $1838,2,450$.

Mobs-Zippe, Min. 2. Aufl. 1839, 2, 149.

Zippe, Verh. d. Ges. d. böhm. Museums 1839, 62.

A. Breith a u t, IIandb. d. Min. $1841,2,194$.

C. Hartmann, Handb. d. Min. 1843, 2, 361 .

G. Leo nhard, Handwörterb. d. topogr. Min. 1843, 91.

II a u s mann, Handb. d. Min. Abth. II, 1847, 2, 1113.

J. Nicol, Manual of Min. 1849, 376.

Sandberger, N. Jahrb. f. Min. etc. 1850, 269 (Ems).

A. Breithaupt, Berg- u. Ilüttenmänn. Zeitung 18:5, 11, 6: (Sierra Almagr.).

Phillips (Brooke a. Miller), Min. 1852, 526.

N. v. Kokscharow, Materialien z. Mlin. Huss. 1853, 1, 34 (Nertschinsk).

Pogg. Ann. 1854, 91, 156.

Dana, Syst. of milo. ed. 2, 1837, ed. 3, 1850.

- - - - ed. 4, 1854, 2, 370.

Kolenali, Dic Mineralien Mährens u. Oeslerr.-Schlesiens etc. 1854.

1. Quenstedt, Handls. d. Min. 1855, 374.

R. Il o fn a n n, Jahrb. d. k. k. geol. Heichsanst. 1855, 6, 1 (Siebenbürgen:

A. Dufrénoy, Traité de Mitl. éd. 1, 1857, 3.

ćd. $2,1858,3,256$.

G. Le onhard, N. Jahrb. f. Min. etc. 1857, 5:50 (Wiesloch).

(ireg and Lettsom, Man. of Min. of Gr. Brit. and Ireland 1854, 247 .

v. Zepharovich, Min. Lex. f. Oesterreich 1859, 16.

J. I. Smith, Erdm. Journ. 1835, 66, $43 \mathrm{~g}$ (Wheatley).

V. v. Lang, Wien. Sitzber. 1859, 36, 241 (Monogr.).

D a uber, Pogg. Ann. 1859, 108, 444.

Schrauf, Wien. Sitzber. 1860, 39, 913.

Hessenberg, Senk. $\Lambda$ bh. 18634,211 (Min. Not. 5, 31).

Quenstedt, Min. 1863, 4 šs.

v. Zepharovich, Wien. Sitzber. 186440 (1), 369 (Schwarzenberg, Mies).

Schrauf, Atlas 1871, Taf. 11 bis 130.

Dana, Syst. $1873,6 z 2$.

v. Zepharovich, Lotos 1874 Hïttenberger Erzberg).

Krenner, diese Zeitschr. 1877, 1, $3 \geq 1$ (Cngarn).

Groth, Strassb. Samml. 1878, 148.

$\left.\begin{array}{c}\text { Q. Sella, Rom Acc. Linc. } 1879(3), 3,450 \\ -\quad \text { diese Zeitschr. } 1880,4,400\end{array}\right\}$ (Sardinien).

$\begin{array}{cl}\text { Jercm eje w, diese Zeitschr. 1883, 7, 637. } \\ \text { - } & \text { Verh. Petersb. min. Ges. 188: 13, 108. }\end{array}$ $\left.\begin{array}{l}\text { Verh. Petersb. min. Ges. 188A, 13, 108. } \\ \text { Jahrb. f. Min. 1883, 2, 329. }\end{array}\right\} \begin{aligned} & \text { Pseudomorphosen von } \\ & \text { Cerussit nach Anglesil. }\end{aligned}$

Franzenau, diese Zeitschr. 1894, 8, :332 (Felsö-Vissó).

Liweh, diese Zeitschr. 1884, 9, 501 (Haus Baden).

Franzenau, diese Zeitschr. $1885,10,88$.

A. Schmidt, diese Zeitschr. 1885, 10, 207 (Pelsöcz-Ardó).

Q. Sella, Rom Acc. Linc. 188: (4), 2, 199-207 (Memor.).

Solly, Min. Mag. a. Journ. of the Min. Soc. 1886 (7), 33, 61 (Portugal).

vom Rath, Sitzber. d. Niederrhein. Ges. f. Naturk. etc. 1887, 130 (Mte. Vecchio).

Bourgeois, Bull. de la Soc. min. de France 1887, 10, 326 (künstlich).

Groth, Zeitschrift f. Krystallogr. XXXIX.

$1888,11,57$ 
Zi ma n yi, Kryst. Unters. 1888, 18, 437 (Peru, Mexico).

V. Goldschmidt, diese Zeitschr. 1890, 18, 287.

Michel, Bull. Soc. min. France 1890, 13, 214.

Dannenberg, diesc Zeitschr. 1890, 18, 64 (Diepenlinchen).

Jeremejew, Verh. Pelersb. Ges. Min. 1892, 29, 193.

Es. Da n a, Syst. $1892,907$.

Williams, diese Zeitschr. 1894, 22, 575 (Union Bridge).

L.acroix, Bull. soc. min. France $1894,17,49$ (Neu-Caledonien).

Compt. rend. 1896, 123, 955 (Laurion, Griechenland).

L ü decke, Die Minerale des Harzes 1896.

Cesàro, Mém. Ac. R. Sciences Belg. 1897, 53, Sept., 1 (Corphalie, Rocbeux).

Butgenbach, Ann. Soc. géol. Belg. 1897, 24, 193 (Neu-Caledonien, Algier).

Spencer, Min. Mag. 1897, 11, 197.

Riva, Rend. Acc. Linc. $1897(\mathbf{5}), 6,426$ (Nebida).

Redlich, Tscherm. min. Mitth. 1898, 17, 518 (Tirol).

de Schulten, Bull. Soc. Min. Fr. 1998, 21, 142 (künstlich).

Millosevich, Riv. Min. Crist. Ital. 1898, 21, 47

Rend. Rom Acc. Linc. 1898, 5, 249

(Malfidano).

K. Jimbo, Journ. Coll. Sci. Tokyo 1899, 11, 213 (Japan).

V. Hul yák, Term. Füz. 1900, 23, 187 (Laurion, Ltah).

Mill o sevich, Riv. Min. Crist. Ital. 1900, 24, 25 (Malfidano!.

- 1903, 30, 23 (Monte Vecchio).

Speciell über Anglesite von Monteponi liegen viele Beobachtungen, Beschreibungen, Messungen und Zeichnungen vor. Die ersten Mitlheilungen über Messungen an Anglesiten von diesem Fundorte finden sich bei Kokscharow (Mat. z. Min. Russl. 1853, 1, 39). Er unterzog einen vorzüglich ausgebildeten Krystall der Messung mit einem Mitscherlich'schen Goniometer mit zwei Fernrohren und berechnete aus derselben die Elemente dieses Minerals. Lang hat diese Kokscharow'schen Elemente mit seinen Messungen verglichen (vgl. S. 262) und dieselben als die zuverlässigsten der Berechnung seiner Winkel zu Grunde gelegt. Er selbst hat, wie schon crwähnt, eine Reihe von Krystallen von Monteponi gemessen, berechnet und beschrieben. Weitere Publicationen über Anglesite von Monteponi verdanken wir Hessenberg (Senk. Abh. 1863, 4, 211); Schrauf (Atlas d. Kryst.-Formen 1871) und Q. Sella (Rom. Acc. Linc. 1879, 3, 150).

Wenn es trotzdem werthvoll erschien, die krystallographische Untersuchung und Beschreibung der Anglesite dieses Fundortes von Neuem in Angriff zu nehmen, so waren hierzu folgende Gründe massgebend:

1. Bei einem Vorkommen von so glänzender Ausbildung, solchem Formenreichthum und Wechsel in Habitus und Ausbildung erschien es nicht nur möglich, sondern wahrscheinlich, dass sich neue Combinationen, auch wohl neue Formen würden nachweisen lassen. Dies um so mehr, als sehr reiches Material zur Verfügung stand, das von neuerer Gewinnungszeit herrührt (1890-1900), während die Beschreibungen von Sella sich auf Funde vor 1879, die von Schrauf auf solche vor 1871, die von 
Hessenberg auf Material vor 1863 und die von Lang auf solches aus den Zeiten bis zu 1859 beziehen. Ueberdies sind Habitus und Ausbildung bei derselben Grube nicht constant geblieben. Daher künnen neue Anbrüche jederzeit auch neue Erscheinungen zu Tage bringen.

2. Die zweikreisige Messung und die Vervollkommungen der Instrumente machten es möglich, Messungen auszuführen, die früher nicht müglich waren.

3. Es traten in den Bereich der Untersuchungen auch die krummen Flüchen wohlausgebildeter Krystalle (Uebergangs(lächen) 1). Ja, neueste Untersuchungen haben gezeigt, dass die Reflexe der krummen Flächen in den Hauptzonen der. Formenentwicklung liegen und dass es möglich ist, aus ihnen das Formensystem der Krystallart in seinen Hauptzügen abzuleiten. Es schien wichtig, mit Hülfe der Anglesitkrystalle einen Beitrag zu dieser Frage zu liefern. Ifierzu boten eigenthümliche, gekrümmte Flächen, die sich an dem Materiale fanden, Gclegenheit.

4. Die publicirten Krystallfiguren des Anglesits geben idealisirte Bilder. Ausnahme bilden nur die zehn von Lang (vgl. S. 274-289) gegebenen Kopfbilder und die perspectivischen Bilder auf Tafel VII, Fig. 44-49. Es erschien aber von Interesse, die Krystalle im Kopf- und perspectivischem Bilde müglichst genau in den Verhältnissen abzubilden, wie sie die Natur hervorgebracht hat.

כ̈. Neuere Untersuchungen über die Entwickelung der Krystallformen und Discussion der Reihen der Symbolzahlen machten es möglich, zweifelhafte Formen auf ihre Wahrscheinlichkeit zu prüfen und somit zur Klärung des Verzeichnisses der typischen Formen beizutragen.

6. Die gross angelegte Untersuchung Q. Sella's über den Anglesit von Sardinien wurde leider nie zu Ende geführt. Sie wurde 1853 begonnen; 1879 aber sah sich der ausgezeichnete Mineralog und Staatsmann, dessen Indenken Denkmale in Rom, Turin und auf Sardinicn ehren, genöthigt, statt der ausführlichen Publication, die er in Folge anderweitiger wichtiger Geschäfte nicht mehr hoffen konnte zu vollenden, sie in einer nur wenige Seiten umfassenden Mittheilung zu publiciren, die den Charakter einer vorlïufigen Mittheilung hat. Sie enthielt nicht viel mehr als die Aufzählung von 12 neuen Formen mit den nothdürftigsten Messungsangaben, ohne Figuren und ohne nühere Beschreibung der Formen.

Sella bemerkt selbst: »dass manche dieser Symbole, sowohl wegen ihrer Ausbildung, als wegen der Natur der Formen, auf die sie sich beziehen, nicht als definitiv gesichert angesehen werden können. - Er behält sich vor, am Ende seiner Untersuchungen zu erklären, welche Symbole

1) Vgl. Goldschmidt, Ceber krumme Flächen. Diese Zeitschr. 1896, 26, 1. 
als sicher anzusehen sind, welche als wahrscheinlich, und welche zu beseitigen sind $x$.

Es erschien von Interesse und Wichtigkeit, die Publication von Sella durch das, was ihr fehlt, nämlich durch Abbildungen und Specialbeschreibungen, zu ergänzen.

\section{Beobachtete Formen.}

Aufstellung, Buchstabenbezeichnung, sowie die zur Berechnung dienenden Elemente wurden nach Goldschmidt's Winkeltahellen angenommen. An den dieser Arbeit zu Grunde liegenden Krystallen fanden sich folgende Formen:

\begin{tabular}{|c|c|c|c|c|}
\hline \multirow{2}{*}{ Nr. } & \multirow{2}{*}{ Buchst. } & \multicolumn{3}{|c|}{ Symbole } \\
\hline & & Miller & Naumann & Goldsch midt \\
\hline 1 & $c$ & $\{001\}$ & $0 P$ & 0 \\
\hline 2 & $a$ & $\{010\}$ & $\infty \breve{P}_{\infty}$ & $0 \infty$ \\
\hline 3 & $b$ & $\{100\}$ & $\infty \bar{D} \infty$ & $\infty 0$ \\
\hline 4 & $N$ & $\{310\}$ & $\infty \bar{p} 3$ & $3 \infty$ \\
\hline 5 & $\lambda$ & $\{210\}$ & $\infty \bar{P}_{2}$ & $2 \infty$ \\
\hline 6 & $* Q$ & $\{: 30\}$ & $\infty P^{5}$ & $\frac{5}{4} \infty$ \\
\hline 7 & $m$ & $\{110\}$ & $\infty P$ & $\infty$ \\
\hline 8 & $h$ & $\{340\}$ & $\infty \vec{P}_{i}$ & $\infty_{3}^{1}$ \\
\hline 9 & $n$ & $\{120\}$ & $\infty P \mathscr{P}$ & $\infty 2$ \\
\hline 10 & $x$ & $\{130\}$ & $\infty \breve{P}$ & $\infty 3$ \\
\hline 11 & $o$ & $\{011\}$ & $\bar{P} \infty$ & 01 \\
\hline 12 & $\boldsymbol{G}$ & $\{108\}$ & $\bar{P} \infty$ & $\frac{1}{8} 0$ \\
\hline 13 & $l$ & $\{104\}$ & $\bar{p} \infty$ & 10 \\
\hline 14 & $d$ & $\{102\}$ & $i \overline{2} \infty$ & $\frac{1}{2} 0$ \\
\hline 15 & $r$ & $\{112\}$ & $P$ & $\frac{1}{2}$ \\
\hline 16 & $\approx$ & $\{111\}$ & $P$ & 1 \\
\hline 17 & $\boldsymbol{\tau}$ & $\{221\}$ & $2 P$ & 2 \\
\hline 18 & $y$ & $\{122\}$ & $\breve{p q}$ & $\frac{1}{2} \cdot 1$ \\
\hline 19 & $\zeta$ & $\{142\}$ & $2 \breve{P}_{4}$ & $\frac{1}{2} 2$ \\
\hline 20 & $u$ & $\{124\}$ & $1 \breve{p q}$ & $+\frac{1}{2}$ \\
\hline 21 & $p$ & $\{324\}$ & $\ddot{p}_{3}$ & $\frac{3}{4}$ \\
\hline 22 & ?) 9 & $\{342\}$ & $2 \overrightarrow{P 3}$ & $\frac{3}{2} \overline{2}$ \\
\hline 23 & $* \eta$ & $\{125\}$ & $2 \overline{p q}^{2}$ & $-\frac{2}{5}$ \\
\hline 24 & c & $\{126\}$ & $\frac{1}{3} \mathbf{p} 2$ & $\frac{1}{3}$ \\
\hline $2: 3$ & $* \Sigma$ & $\{127\}$ & $\frac{2}{7}$ & $\frac{1}{7} \frac{2}{7}$ \\
\hline 26 & $w$ & $\{128\}$ & $\frac{i}{4} \not 2$ & $\frac{1}{6} \frac{1}{4}$ \\
\hline
\end{tabular}

Die mit * bezeichneten Formen sind für Anglesit neu. ?) Vgl. S. 473.

\section{Beschreibung der einzelnen Krystalle.}

In der Art der Bezeichnung der Einzelflächen bin ich dem Vorschlage Goldschmidt's gefolgt (Index Einl. S. 143), die Einzelflächen mit Ziffern zu bezeichnen, die den Quadranten angeben, in dem die Fläche liegt.

So bedeutet $r^{2}$ die Fläche $r$ im zweiten Quadranten der oberen Krystallhälfte.

Bei den Flächen der unteren Krystallhälfte wurde der, den Quadranten angebende Index unten an den Buchstaben angehängt und zwar so, dass 
$r_{2}$ die Gegenfäche von $r^{2}$ bezeichnet. In den Figuren wurden diese Indices weggelassen, ebenso die Buchstaben für die hinteren Flächen in den perspectivischen Bildern, da bier die Bedeutung der hinteren Flichen ohne Weiteres aus den beigegebenen Kopfbildern ersichtlich sind.

Das vorliegende Material liess sich in drei Typen eintheilen, je nachdem die Streckung des Krytalles nach der einen oder anderen oder dritten Axe erfolgte.

I. Typus. Durch die Streckung der $c$-Axe erlangt der Krystall einen prismatischen Habitus nach der Prismenzone.

Diesem Typus gehört eine Reihe der beobachteten Krystalle an, die einander oft ähnlich sind und meistens die Basis $c=0$ entwickelt haben.

Krystall 1 (Taf. XI, Fig. 1ab). Ein wunderbar klarer, glattflächiger, nur an der Basis und der einen Seite etwas ausgebrochener Krystall mit den $\Lambda$ bmessungen $7 \times 6 \times 4 \mathrm{~mm}$. Er zeigt die Formen:

$\begin{array}{cccccccccccccc}c & b & \lambda & m & n & x & o & l & d & r & z & \tau & y & p \\ 0 & 0 \infty & 2 \infty & \infty & \infty 2 & \infty 3 & 01 & \frac{1}{4} 0 & \frac{1}{2} 0 & \frac{1}{2} & 1 & 2 & \frac{1}{2} 1 & 3 \frac{1}{2}\end{array}$

mit den Einzelflächen:

$e^{1} \ldots b^{1} \ldots \lambda^{4} \ldots m^{1} m^{2} m^{4} \ldots n^{4} \ldots{\varkappa^{4}}^{4} \ldots o^{1} \ldots l^{1} l^{2} \ldots$

$d^{1} \ldots r^{1} r^{2} r^{4} \ldots z^{1} z^{2} z^{4} \ldots \tau^{1} \tau^{2} \tau^{4} \ldots y^{1} y^{2} \ldots p^{1} p^{2}$

Die Reflexe sind einfach und gut, bis auf denjenigen der Fläche $\lambda^{4}$, welche eine Streifung $\|$ der Kante $\lambda / m$ trägt. An diesem Krystalle fand ich eine kleine, gut reflectirende Fläche neben einer einspringenden Kante, welche folgende Positionswinkel hatte: $\varphi=\pi 73056^{\prime} ; o=51045^{\prime}$. Die dieser Form am nächsten stehende bekannte Form ist $x=\frac{1}{12} 1$ (1.12.12), (k, Fran-

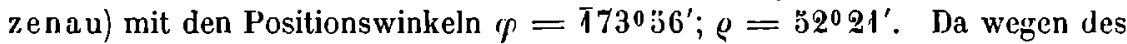
Anliegens am einspringenden Winkel eine Beeinflussung durch die Nachbarllächen (Influenzirung) sehr wohl müglich war, so wurde, besonders im Hinblick auf die Complication des Symbols, die Form nicht als typisch angesehen.

Krystall 2 (Taf. XI, Fig. 4 ab). Ein ebenso schüner Krystall wie der vorige, mit den $\Lambda$ lomessungen $7 \times 6 \times 5, ; 3 \mathrm{~mm}$ und fast durchgehend gut reflectirenden Flächen. Nur $m$ und $a$ zeigen Lüngsstrcifungen \| der Kante $a / m$ und geben daher mehrfache Reflexe. Beobachtet wurden die Formen:

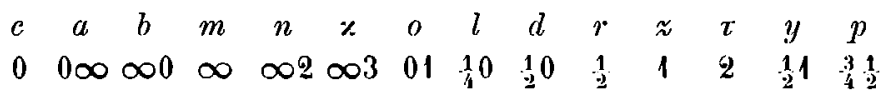

mit den Einzelflichen:

$c^{1} \ldots a^{1} \ldots b^{1} b^{2} \ldots m^{1} m^{3} m^{4} \ldots n^{1} n^{3} n^{4} \ldots x^{1} \varkappa^{4} \ldots l^{1} \ldots$ $d^{1} d^{2} \ldots r^{1} r^{2} r^{4} \ldots \approx^{1} z^{2} z^{3} z^{4} \ldots \tau^{1} \tau^{2} \tau^{3} \tau^{4} \ldots y^{1} y^{2} y^{4} \ldots p^{1} p^{2} p^{3}$.

Krystall 3 (Taf. XI, Fig. 6ab) ist von ähnlicher Auslildung und Be- 
schaffenheit wie die vorangehenden und hat die Abmessungen $4, \tilde{a} \times 4 \times 3 \mathrm{~mm}$. Er zeigte nachstehende Formen:

$$
\begin{array}{ccccccccccccc}
c & a & b & m & n & o & l & d & r & \approx & \tau & y & p \\
0 & 0 \infty & \infty 0 & \infty & \infty 2 & 01 & 1.0 & 10 & 1 & 1 & 2 & 1.1 & 31.1
\end{array}
$$

mit folgenden Einzelflächen:

$$
\begin{gathered}
c^{1} \ldots a^{2} \ldots b^{1} b^{2} \ldots m^{1} m^{2} m^{3} m^{4} \ldots n^{2} n^{3} n^{4} \ldots o^{1} o^{2} \ldots \\
l^{1} l^{2} \ldots d^{2} \ldots r^{1} r^{2} r^{3} r^{4} \ldots z^{1} z^{2} z^{3} z^{4} \ldots \tau^{1} c^{2} r^{3} \ldots \\
y^{1} y^{2} y^{3} y^{4} \ldots p^{1} p^{2} p^{3} p^{4} .
\end{gathered}
$$

Krystall 4 (Taf. XI, Fig. 3ab). Ein wasserklarer, an einigen Stellen angebrochener, bez. überwachsener, sonst glattflächiger Krystall mit den Abmessungen $4 \times 3 \times 2 \mathrm{~mm}$ und den Formen:

$$
\begin{aligned}
& \begin{array}{llllllllllllllll}
c & a & b & \Omega & m & h & n & x & o & l & d & r & \approx & \tau & y & p
\end{array}
\end{aligned}
$$

\begin{tabular}{|c|c|c|c|c|c|}
\hline \multirow{2}{*}{ Buchst. } & \multicolumn{2}{|c|}{ Gemessen } & \multicolumn{2}{|c|}{ Berechnet } & \multirow{2}{*}{ Bemerkungen } \\
\hline & $\varphi$ & 0 & $\boldsymbol{\varphi}$ & 0 & \\
\hline 91 & $57^{0} 14^{\prime}$ & $9000^{\prime}$ & $37052^{\prime}$ & $9: 00^{\prime}$ & Fläche gestreift; Reflex mebrfach \\
\hline 92 & 12141 & > & 1228 & » & schwach \\
\hline$\underline{3}$ & $\overline{1} 21 \quad 59$ & $\infty$ & 1228 & , & ziemlich gut \\
\hline
\end{tabular}

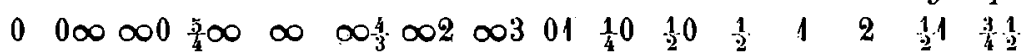

und den Einzelflächen:

$$
\begin{gathered}
c^{1} \ldots a^{1} \ldots b^{1} b^{2} \ldots \Omega^{1} \Omega^{2} \Omega^{3} \ldots m^{1} m^{2} m^{3} m^{4} \ldots h^{4} \ldots \ldots \\
n^{4} \ldots x^{4} \ldots o^{1} o^{2} \ldots l^{1} l^{2} \ldots d^{1} d^{2} \ldots r^{1} r^{2} r^{4} \ldots z^{2} z^{2} z^{3}{ }^{4} \ldots \ldots \\
\tau^{3} \tau^{4} \ldots \ldots y^{1} y^{2} y^{3} y^{4} \ldots p^{1} p^{2} p^{3} p^{4} .
\end{gathered}
$$

Neu ist das Prisma $\Omega=\frac{5}{4} \infty 0(540)$. Es fand sich am Krystalle mil folgenden Flächen:

Die Abweichungen der Winkel der Beobachtung in den beiden ersten Fällen gegenüber der Rechnung dürfte wohl auf die ungünstige Beschaffenheit der Flächen zurückzuführen sein; hingegen stimmt die Messung von $\Omega^{3}$ mit der Berechnung ziemlich überein. Die Form wurde als gesichert angesehen. Für sie spricht der Umstand, dass sie mit drei von vier zusammengehürigen Flächen auftritt.

Krystall 5 (Taf. XI, Fig. 5ab). Ein ebenfalls wasserklarer, fast unbeschädigter Krystall mit meist gut reflectirenden Flächen; nur $N$ zeigte eine geringe Streifung $\|$ der Kante $m / N$. Da $N$ bis jetzt. nur von Jeremejew an Pseudomorphosen einmal beobachtet worden ist, so will ich die Messungsresultate für diese Fläche anführen. Durch die Streifung erhielt ich einen dreifachen Reflex: 


\begin{tabular}{|c|c|c|c|c|c|c|}
\hline & & \multicolumn{2}{|c|}{ Gemossen } & \multicolumn{2}{|c|}{ Berechnct } & \multirow{2}{*}{$i$} \\
\hline & & $\varphi$ & 9 & $\boldsymbol{p}$ & $\rho$ & \\
\hline \multirow{3}{*}{ Rellex } & 1 & $\overline{7} 5015$ & $\operatorname{coO}_{v^{\prime}}$ & $\overline{7}: 0 \pm J^{\prime}$ & $9000^{\prime}$ & $\lambda^{4}$ \\
\hline & 2 & $\overline{7} \bar{z} 2: 3$ &. & & & \\
\hline & 3 & $\overline{7} 538$ & > & & & \\
\hline
\end{tabular}

Die Messungen weichen selır geringlügig (besonders Reflex 1 und 2) von der Berechnung ab, so dass dic For'm genau identificirt werden konnte. Der Krystall hat die Abmessungen $8 \times \ddot{3} \times 3 \mathrm{~mm}$ und folgende Formen:

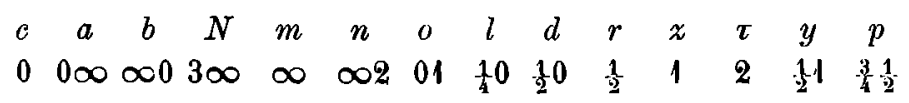

mit den Einzelflächen:

$$
\begin{gathered}
d^{1} \ldots a^{1} a^{2} \ldots b^{1} \ldots N^{4} \ldots m^{1} m^{2} m^{3} m^{4} \ldots n^{2} n^{4} \ldots \\
o^{1} o^{2} \ldots l^{1} \ldots d^{1} d^{2} \ldots r^{3} \ldots z^{1} z^{2} z^{3} z^{4} \ldots \ldots \tau^{1} \tau^{2} \tau^{3} z^{4} \ldots \ldots \\
y^{1} y^{2} y^{3} y^{4} \ldots \ldots p^{1} p^{3} p^{4}
\end{gathered}
$$

Krystall 6 (Taf. XII, Fig. 16ab). Ein durch seine viermal auftretenden. Krümmungen und eine eigenartige Wachsthumserscheinung bemerkenswerther Krystall mit den Abmessungen $5 \times 5 \times 3,5 \mathrm{~mm}$. Die Wachsthumserscheinung iussert sich in folgender Weise: Ein innerer, milchig getrübter Krystallkern ist von einem spüter gebildeten, wasserhellen Krystall umwachsen; und zwar so, dass der erste stellenweise noch aus dem zweiten hervorragt. Beide Krystalle haben die Basis $c=0$ gemeinsam, nur ein kleiner Teil des Kernes tritt aus dem l-Doma hervor und wird hier von folgenden Flächen begrenzt: 先 $\ldots p^{1} p^{2} \ldots r^{1} r^{2}$ und $d^{1}$. Die Formen sind bis auf $d$ am Umhüllungskrystalle überhaupt nicht ausgebildet. Die untere Krystallhälfte war an einer kleinen Stelle aufgewachsen und beim Loslösen aus der Stufe kaum nennenswerth beschädigt worden, so dass ich in der Lage war auch sie zu messen. An ihr fanden sich die neven Formen $\eta$ und $\mathbb{\Xi}$ und die nur einmal von Sella beobachtete c-Fläche. Es wurden im Ganzen an diesern Krystalle folgende Formen festgestellt:

$$
\begin{aligned}
& \begin{array}{lllllllllllllll} 
& b & m & o & l & d & r & \approx & y & \boldsymbol{l} & p & \eta & \mathbf{c} & \mathbf{5} & w
\end{array}
\end{aligned}
$$

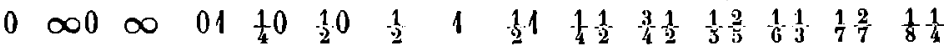

deren Einzelflïchen sich folgendermassen verteilen:

auf die Krystalloberhälfte, ausschliesslich des Kernkrystalles:

$$
\begin{gathered}
c^{1} \ldots b^{1} b^{2} \ldots \ldots m^{1} m^{2} m^{3} m^{4} \ldots o^{1} o^{2} \ldots l^{1} l^{2} \ldots d^{1} d^{2} \ldots \\
y^{1} y^{2} y^{3} y^{4}
\end{gathered}
$$

auf den Krystallkern:

$$
c^{1} \ldots d^{1} \ldots r^{1} r^{2} \ldots \approx^{1} \ldots p^{1} p^{2}
$$

und auf die Unterhälfte: 
$c_{1} \ldots b^{1} b^{2} \ldots \ldots m^{1} m^{2} m^{3} m^{4} \ldots l_{1} l_{2} \ldots d_{1} d_{2} \ldots y_{1} y_{2} y_{3} y_{4} \ldots$ $\mu_{2} \mu_{3} \ldots \eta_{2} \eta_{3} \ldots c_{3} \ldots \Sigma_{3} \ldots w_{2}$.

$\eta=\frac{1}{5} \frac{2}{5}(125)$ ist eine neue Form für den Anglesit. Von ihr fanden sich zwei Flächen $\eta_{2}$ und $\eta_{3}$ vor. Beide Flächen waren klein und mussten mit Verkleinerung und Abblendung gemessen werden, wobei sie scharfe Reflexe gaben. Da Messung und Kechnung ziemlich gut übereinstimmen, ausserdem die Symbolzahlen, wie auch die der folgenden Form, gut in die Zahlenreihen (vgl. Discussion) des Anglesits passen, so dürfte $\eta$ als gesichert gelten.

$\Sigma=\frac{1}{7} \frac{2}{7}$ (127) ist ebenfalls eine neue Form. Sie wurde mit nur einer Fläche beobachtet und zwar im einspringenden Winkel, so dass an Influenzirung durch Nachbarflächen wohl gedacht werden konnte. Doch ist diese Fläche leidlich gross und scharf, ausserdem im Zonenverbande mit $\mu_{3}, \eta_{3}$ und $\mathfrak{c}_{3}$ ausgebildet; sodann stimmen Messung und Berechnung gut überein, so dass wir wohl berechtigt sind, die Form als typisch anzusehen. Die Form $\mathfrak{c}=\frac{1}{6} \frac{1}{3}(126)$ wurde bis jetzt nur einmal von Sella beobachtet. Aus diesem Grunde füge ich meine Messungsresultate dieser Form den nachstehenden von $\eta$ und $\Sigma$ bei.

\begin{tabular}{|c|c|c|c|c|c|c|c|c|}
\hline \multirow{2}{*}{ Buchst. } & \multicolumn{2}{|c|}{ Gemessen } & \multicolumn{2}{|c|}{ Berechnet } & \multirow{2}{*}{\multicolumn{4}{|c|}{ Bemerkungen }} \\
\hline & $\boldsymbol{p}$ & $\varphi$ & $\varphi$ & $\varrho$ & & & & \\
\hline $\begin{array}{l}r_{2} \\
r_{3}\end{array}$ & $\left|\begin{array}{rrr}14 & 7029^{\prime} \\
14 & 31\end{array}\right|$ & $\begin{array}{l}300.39^{\prime} \\
31 \quad 18\end{array}$ & $\mid \begin{array}{l}147031 \\
14731\end{array}$ & $\begin{array}{c}31026^{\prime} \\
?\end{array}$ & Fläche & $\begin{array}{l}\text { klein, schmal; } \\
\text { ein ki. Dreieck; }\end{array}$ & Refl. & $\begin{array}{l}\text { schwach } \\
\text { gut }\end{array}$ \\
\hline $\mathbf{C}_{3}$ & > & 274 & > & 270 & s & ziemlich gross; & , & s \\
\hline$\Sigma_{3}$ & s & 2341 & 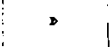 & 2335 & . & 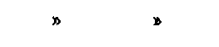 & , & 3 \\
\hline
\end{tabular}

Krystall 7 (Taf. XI, Fig. 2ab). Dieser Krystall zeichnet sich durch die vorzugsweise Ausbildung der Pinakoide aus. Er ist nur an der Wurzel abgebrochen und mit mehreren kleineren Kryställchen von gleicher Combination und gleichem Habitus durch Parallelverwachsung verknüpft. Die Flächen sind glatt und reflectiren gut. Seine Grüsse beträgt $6 \times 4,5 \times 4 \mathrm{~mm}$. Es wurden folgende Formen beobachtet:

$$
\begin{array}{ccccccccc}
c & a & b & m & o & r & z & y & p \\
0 & 0 \infty 0 & \infty 0 & \infty & 01 & \frac{1}{2} & 1 & \frac{1}{2}-1 & \frac{3}{4} \frac{1}{2}
\end{array}
$$

mit den Einzelflächen:

$$
\begin{gathered}
c^{1} \ldots a^{1} a^{2} \ldots b^{1} b^{2} \ldots m^{1} m^{2} m^{3} m^{4} \ldots a^{1} o^{2} \ldots r^{1} r^{2} r^{3} r^{4} \ldots \\
z^{1} \ldots y^{1} y^{2} y^{3} y^{4} \ldots p^{1}
\end{gathered}
$$

Krystall 8 (Taf. XII, Fig. 15 ab). Durch die Entwickelung eines Pinakoides $(b=\infty 0)$ und die Streckung eines Domas $(0=01)$ erhält der Krystall bei tafeligem Habitus eine barytähnliche Gestalt. Der Krystall ist wasser- 
klar und zeigt eine deutliche Spaltbarkeit nach der Basis $c=0 . \quad \operatorname{Er}$ hat die $\Lambda$ bmessungen $10,3 \times 8 \times 3,5 \mathrm{~mm}$ und liess nachstehende Formen erkennen:

$$
\begin{array}{cccccccc}
c & b & m & n & o & d & z & y \\
0 & \infty 0 & \infty & \infty 2 & 01 & \frac{1}{2} 0 & 1 & \frac{1}{2} 1
\end{array}
$$

mit den Einzelflächen:

$$
\begin{gathered}
c^{1} \ldots b^{1} b^{2} \ldots m^{2} m^{3} \ldots n^{1} n^{2} n^{4} \ldots o^{1} o^{2} \ldots a^{1} d^{2} \ldots \\
z^{2} z^{4} \ldots y^{2} y^{4} .
\end{gathered}
$$

Krystall 9 (Taf. XII, Fig. 18ab). Dieser Krystall kommt in verschiedenen Beziehungen dem vorangehenden nahe. $\mathrm{Er}$ ist jedoch mit der einen $b$-Fläche aufgewachsen, auch zeigen die Pyramiden $y$ eine grüssere Breitenentwickelung. Seine Abmessungen belragen $8 \times 7 \times 4,5 \mathrm{~mm}$ und er zeigt folgende Formen:

$$
\begin{array}{cccccccc}
e & b & o & d & r & z & y & p \\
0 & \infty 0 & 01 & \frac{1}{2} 0 & \frac{1}{2} & 1 & \frac{1}{2} 1 & \frac{3}{4} \frac{1}{2}
\end{array}
$$

mit den Einzelf ächen:

$$
\begin{gathered}
c^{1} c^{2} \ldots b^{2} \ldots o^{2} o^{3} o_{1} o_{4} \ldots d^{2} d_{1} \ldots r^{2} \ldots z^{2} z^{3} z_{1} \ldots \\
y^{1} y^{2} y^{3} y^{4} y_{1} y_{2} y_{3} y_{4} \ldots p_{1} .
\end{gathered}
$$

Die $b$-Fläche zeigt eine Streifung $\|$ einer (nicht entwickelten) Kante $b / m$. Ausserdem ist eine konische Auftreibung sämmtlicher $y$-Flächen zu bemerken.

Krystall 10 (Taf. XII, Fig. 17 a, b, c). Dieser Krystall zeigt als einfachste Combination nur das Prisma $m=\infty$, das Doma $d=\frac{1}{2} 0$ und das Pinakoid $b=\infty 0$; letzteres ist nur einmal mit einer ganz schmalen Fläche vertreten. Der Krystall hat folgende Abmessungen: $15 \times 9 \times 8 \mathrm{~mm}$, und die Einzelflächen:

$$
b^{2} \ldots m^{1} m^{2} m^{3} m^{4} \ldots d^{1} d^{2} d_{1} d_{22} .
$$

Die $d$-Flächen tragen an den Kanten $m / d$ charakteristische Krümmungen. Ueberdies ist eine eigenthümliche Wachsthumserscheinung sichtbar, die in den angeführten Figuren dargestellt ist. Die $d$-Flächen zeigen nämlich je eine dreieckige Vertiefung, nach welcher krumme Flächentheile verlaufen, deren Reflexe sich im Allgemeinen mit denen der $m / d$-Kantenkrümmungen decken, mithin ebenfalls in der $m / d$-Zone liegen.

II. Typus. Die Krystalle besitzen einen domatischen Habitus, welcher durch die Streckung der Makrodomen nach der $l / d$-Kante hervorgerufen worden ist.

Krystall 11 (Taf. XI, Fig. 7). Dieser, sowie der folgende Krystall, ist durch das Vorherrschen der l-Domen sehr flach ausgebildet. Er hat die Abmessungen $9,5 \times 7 \times 2 \mathrm{~mm}$ und zeigt nachstehende Combination: 
mit den Finzelflächen:

$$
\begin{aligned}
& \begin{array}{lllllll}
c & b & m & G & l & y & w
\end{array} \\
& 0 \infty 0 \infty \quad \frac{1}{8} 0 \frac{1}{4} 0 \frac{1}{2} 1 \frac{1}{8} \frac{1}{4}
\end{aligned}
$$

$$
e^{1} c_{2} \ldots b^{1} b^{2} \ldots m^{1} m^{2} \ldots G^{2} G_{4} \ldots l^{1} l^{2} l_{3} l_{4} \ldots y^{1} y^{2} y_{3} y_{4}
$$

Zwischen $l^{1}$ und $G^{2}$, und $G^{2}$ und $l^{2}$ liegen eine Anzahl Riefen, deren lieflexe auf die seltenen und unsicheren Formen $K, E, F, J, K$ hinzudeuten scheinen, doch liessen sich hier keine typischen Formen feststellen. Da $G$ bis jetzt nur von Sella beobachtet wurde, so halte ich es für angezeigt, an dieser Stelle meine Messungsresultate für diese Form anzuführen.

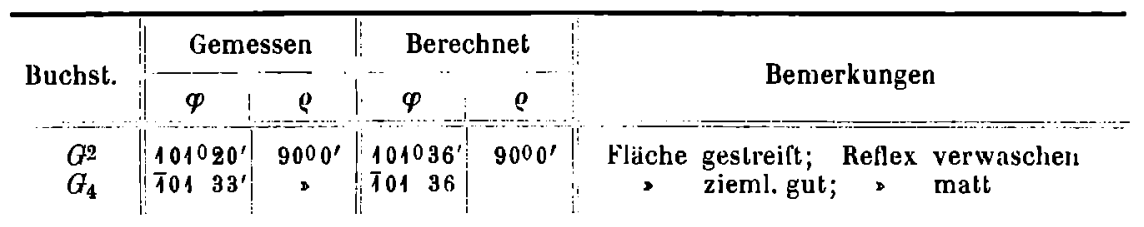

Die übrigen Flächen, mit Ausnahme der facettirten $m$-Flächen, geben gute Reflexe.

Krystall 12 (Taf. XI, Fig. 8a, b). Die $l$ - und $d$-Flächen sind hier der Kanle $d / l$ gestreift. Der Krystall hat die Abunessungen $6 \times 5 \times 1,5 \mathrm{~mm}$ und folgende Formen:

$$
\begin{aligned}
& \begin{array}{lllllllllll}
c & a & b & m & o & l & d & \approx & y & \|
\end{array}
\end{aligned}
$$

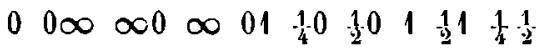

und der Einzelliiichen:

$$
{ }^{1} c_{2} \ldots a_{1} \ldots b^{1} b^{2} \ldots m^{1} m^{2} \ldots o^{1} o_{2} \ldots l^{1} l^{2} l_{3} l_{4} \ldots d_{3}
$$

Krystall 13 (Taf. XI, Fig. 9a, b). Dieser und die nächsten drei Krystalle zeichnen sich durch die vorzugsweise Entwickelung der $d$-Flächen aus. Fr hat die Abmessungen $7,5 \times 7 \times 5,5 \mathrm{~mm}$ und nachstehende Formen:

mit den Einzelflächen:

$$
\begin{array}{cccccc}
c & b & o & d & y & \mu \\
0 & \infty 0 & 01 & \frac{1}{2} 0 & \frac{1}{2}-1 & \frac{1}{4} \\
0
\end{array}
$$

$$
c_{1} \ldots b^{2} \ldots o^{1} o_{2} \ldots d^{1} d^{2} d_{3} d_{4} \ldots y^{1} y_{3} \ldots \mu^{1} \mu^{2} \mu_{3} \mu_{4} .
$$

Die $o$-Flächen zeigen starke sattelfürmige Krümmungen. Die übrigen Flïchen geben, trotz der betrïchtlichen Beschädigungen der $d$-Flächen, gute Reflexe.

Krystall 14 (Taf. XI, Fig. 10a, b). Dieser Krystall zeigt eine deutliche Spaltbarkeit nach dem Prisma $m=\infty$. Er hatte folgende Formen ansggelildet: 


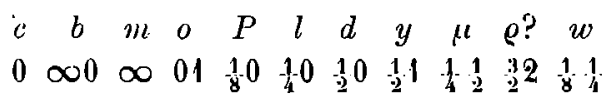

Einzelflïchen waren folgende vorhanden:

$c^{1} c_{1} \ldots b^{2} \ldots m^{1} m^{2} \ldots o^{1} o_{2} \ldots G^{2} \ldots l^{1} l^{2} l_{3} l_{4} \ldots$ $d^{1} d^{2} d_{3} d_{4} \ldots y^{1} y^{2} y_{3} y_{4} \ldots \mu^{1} u^{2} u_{3} \ldots \ldots\left[\varrho^{1} \varrho^{2} \varrho_{3} \varrho_{4}\right] ? \ldots w^{1} w^{2} w_{3} w_{4}$.

Zwischen $l^{1}$ und $l^{2}$ liegt cine Reihe Riefen, die nur durch die Flächen $e^{1}$ und $G^{2}$ unterbrochen wird. $\Lambda$ uch an dieser Stelle müchte ich dic Messungen für $G$ anführen.

\begin{tabular}{c|cc||ccc}
\hline & \multicolumn{2}{c}{ Gemessen } & \multicolumn{2}{c}{ Berechnet } & Bemerkungen \\
Buchst. & $\varphi$ & $\varrho$ & $\frac{\varphi}{\varphi}$ & $\varrho$ & \\
\hdashline$G^{2}$ & $101043^{\prime}$ & $9000^{\prime}$ & $101036^{\prime}$ & $9000^{\prime}$ & Flaiche schmal; Reflex gut
\end{tabular}

Unter den Rellexen der erwähnten Riefen befanden sich einige, die müglicher Weise auf die bei Krystall 11 angegebenen unsicheren Formen hinweisen, doch konnten keine typischen Flächenarten festgestellt werden. Bezüglich der $\varrho$ ?-Fläche möchte ich erwähnen, dass die gemessenen Winkel von den berechneten um mehr als 10 zum Theil abweichen, so dass es sich müglicher Weise um eine neue Form hier handeln kann. Ich gebe daher die Messungen dieser Flïchen und zum Vergleich die berechneten Winkel für $\varrho$ und für eine besser passende Form $\left(\frac{8}{15} \frac{1}{15}\right)$ an.

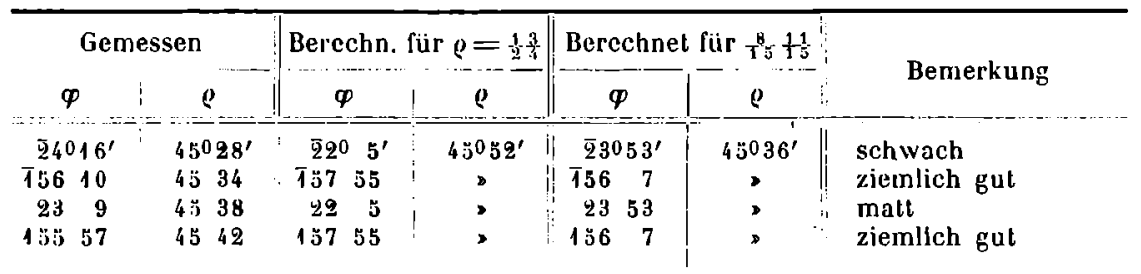

Die neu aufgestellte Form hat in der gewöhnlichen Aufstellung (nach Lang) folgendes Symbol: $\frac{15}{11} \frac{g}{1 T}$ (15.8.11). Bei der ungewühnlichen Complicirtheit dieses Symbols halte ich es für bedenklich, die Form als typisch zu bezeichnen, um so mehr als sich dieselbe bei diesem Krystalle in unmittelbarer Nachbarschaft von bedeutenden Krümmungen befindet. Jie Grösse des Krystalles beträgt 6,5 $56 \times 3 \mathrm{~mm}$.

Krystall 15 (Taf. XII, Fig. $11 \mathrm{a}, \mathrm{b}$ ) ist ein klarer, aber gelblich gefärbter, $6 \times 5 \times 3,5 \mathrm{~mm}$ grosser Krystall mit Krümmungen an den Kanten $o, m, o / b$ und $o / l$. Er wurde von einer Bleiglanzstufe losgebrochen, wo er neben krystallisirtem Schwefel aufsass. Nachstehende Formen wurden an ihm beobichtel: 
mit den Einzelflächen:

$$
\begin{array}{cccccc}
c & b & m & o & l & d \\
0 & \infty & \infty & 01 & 110 & 10
\end{array}
$$

$$
c^{1} c_{1} \ldots b^{1} b^{2} \ldots m^{1} \ldots o^{1} o_{2} \ldots l^{1} l^{2} l_{3} l_{4} \ldots d^{2} .
$$

Krystall 16 (Taf. XII, Fig. 12a, b) ist ein farbloser, klarer, $10 \times 5$ $\times 2 \mathrm{~mm}$ grosser Krystall, der im Inneren parallelflächigen Aufbau erkennen lässt. Er besitzt folgende Formen:

$$
\begin{aligned}
& \begin{array}{llllllllllllllllllllllll}
c & b & m & o & l & d & z & y & \mu & w
\end{array}
\end{aligned}
$$

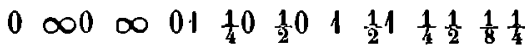

mit den Einzelflächen:

$$
\begin{gathered}
c^{1} \ldots b^{1} b^{2} \ldots n^{1} m^{2} \ldots o^{1} o_{2} \ldots l^{1} l^{2} l_{3} l_{4} \ldots d^{1} d_{3} \ldots z_{3} \\
\ldots \ldots y^{1} y^{2} y_{3} y_{4} \ldots\left\|^{1} u^{2} u_{3}\right\|_{4} \ldots w^{1} w^{2} w_{3} w_{4} .
\end{gathered}
$$

Krystall 17 (Taf. XII, Fig. 13 a,b). Dieser und der folgende Krystall unterscheiden sich von den vorangehenden durch die grössere Entwickelung des Pinakoides $b=\infty 0$. Er hat die Abmessungen $8,5 \times 4 \times 3,5 \mathrm{~mm}$ und zeigt die Combination:

mit den Einzelflächen:

$$
\begin{array}{cccccc}
b & m & o & l & d & y \\
\infty 0 & \infty & 01 & \frac{1}{4} 0 & \frac{1}{2} 0 & \frac{1}{2} 1
\end{array}
$$

$$
b^{1} b^{2} \ldots m^{1} m^{2} \ldots o^{1} o_{2} \ldots l^{2} l_{3} l_{4} \ldots d^{2} d_{3} d_{4} \ldots y^{1} y^{2} y_{3} y_{4} .
$$

Ausserdem besitzt der Krystall Krümmungen an den Kanten $o / m$ und o/l.

K rystall 18 (Taf. XII, Fig. 14 a, b). In Folge des Gleichgewichtes von $b$ und $l$ hat der Krystall einen süulenfürmigen Habitus erlangt. $\mathrm{Er}$ ist wasserklar und lässt im Inneren parallellächigen Aufbau erkennen. Seine Abmessungen betragen $6 \times 2,5 \times 2 \mathrm{~mm}$. Die Flächen waren glatt und gaben gute Reflexe. Es waren nachstehende Formen vertreten:

mit den Einzelflächen:

$$
\begin{aligned}
& \begin{array}{llllllll}
b & o & l & y & \mu & w
\end{array} \\
& \infty 0 \text { 01 } \frac{1}{4} 0 \frac{1}{2} 1 \frac{1}{4} \frac{1}{2} \frac{1}{8} \frac{1}{4}
\end{aligned}
$$

$$
\begin{gathered}
b^{1} b^{2} \ldots o^{1} o_{2} \ldots l^{1} l^{2} l_{3} l_{4} \ldots y^{1} y^{2} y_{3} y_{4} \ldots \mu^{1} \mu^{2} u_{3} !_{4} \\
\ldots \ldots w^{1} w^{2} w_{3} w_{4} .
\end{gathered}
$$

Der Typus III wird durch die Streckung der $b$ - $\Lambda$ xe bedingt. Ihm gehürt nur ein Krystall an, und zwar der

Krystall 19 (Taf. XII, Fig. 19a,b). Derselbe ist klar und farblos, $9 \times 6 \times 4 \mathrm{~mm}$ grass und besilzt die Formen:

$$
\begin{aligned}
& \begin{array}{llllllll}
c & b & m & o & d & y & \zeta
\end{array} \\
& 0 \infty 00 \infty 01 \quad 10 \quad \frac{11}{21}
\end{aligned}
$$


mit den Einzelflächen:

$e^{1} \ldots b_{2} \ldots m^{2} m^{3} \ldots o^{2} o^{3} o_{1} o_{4} \ldots d^{2} d_{1} \ldots y^{2} y^{3} y_{1} y_{4} \ldots \overbrace{}^{33}$.

\section{Statistik über sämmtliche bekannte Combinationen des Anglesits.}

Im Folgenden will ich versuchen, eine Statistik aller bekannten Formen zu geben. Es wurden die in der Literatur aufgefundenen Combinationen, nach Ländern geordnet, zusammengestellt und bei jeder Combination Autor, Fundort, sowie Jahreszahl angegeben. Hierdurch ist man im Stande, mit Hülfc des zu Eingang dieser Arbeit gegebenen Literalurverzeichnisses den Publicationsort und die nähere Beschreibung zu finden.

Ausser der Müglichkeit eines solchen Zurückgehens auf dic Quelle für jede Combination und jede einzelne Flïchenart hat eine solche Statistik noch einen mehrfachen Wert:

Sie ermöglicht, cine Feststellung der relativen Häufigkeit der cinzelnen Formen und dadurch eine liangordnung der Formen in diesem Sinne aufzustellen. Eine Frgünzung dieser Rangordnung crmöglichen die publicirten Bilder, welche die citirten Publicationen begleiten. Die Rangordnung nach JIäufigkeit und Grösse der Ausbildung stellt sich ergäinzend an die Seite der Rangordnung, die sich aus der Entwickelung der Formen im Gesammtbilde, aus Zwillingsgesetzen, Structurflächen") elc. ergiebt. Aus solcher Rangordnung lassen sich die Primärknoten, die uns auf die Richtung der Primürkrïfte der krystallbauenden Partikel führen, ableiten.

Die Buchstaben sind die im Index und in der Winkeltabelle von Goldschmidt gebrauchten, unter Nachtragung der Buchstaben: $D=-\frac{2}{3} 1$ (125) Spencer; $I=13$ (I33) Nillosevich; $C={ }_{7}^{10} \infty(10.7 .0)$ Hulyák; $Y=$ $\frac{2}{9} 0$ (209) Hulyák; $Z=-160(1.0 .16)$ Hulyák; und die, welche für die an meinen Krystallen aufgefundenen neuen formen gewählt wurden: $\Omega=$ to $(540) ; \eta=\frac{1}{3}(125) ; \Sigma=\frac{1}{7} \frac{2}{7}(127)$.

Die Bedeutung der einzelnen Buchstaben kann aus den zum Schlusse angefügten Winkeltabellen entnommen werden.

1) Vergl. Goldschmidt, N. Jahrb. f. Min. etc. 1902, Beil.-Bd. 15, 589 flgde. 


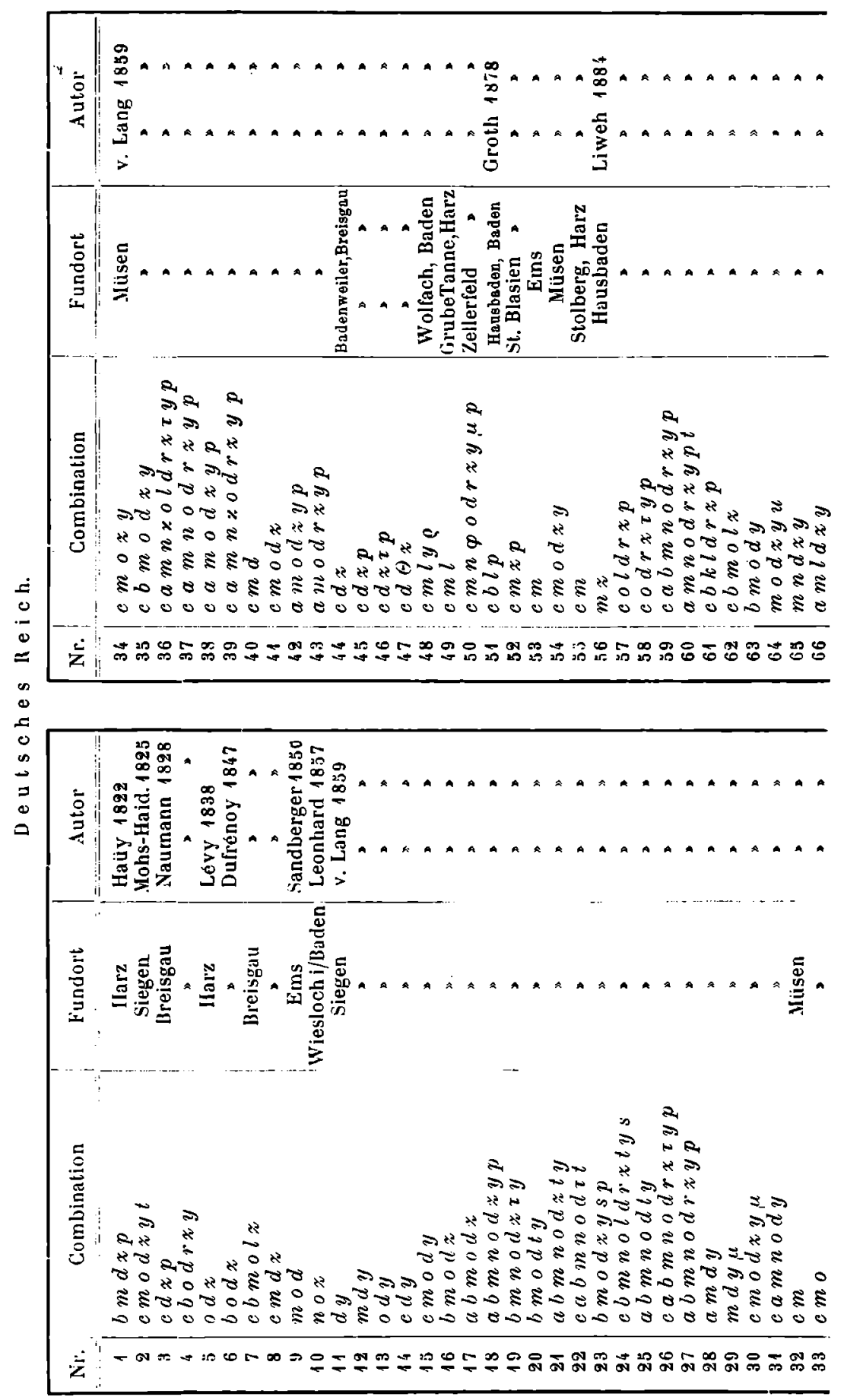



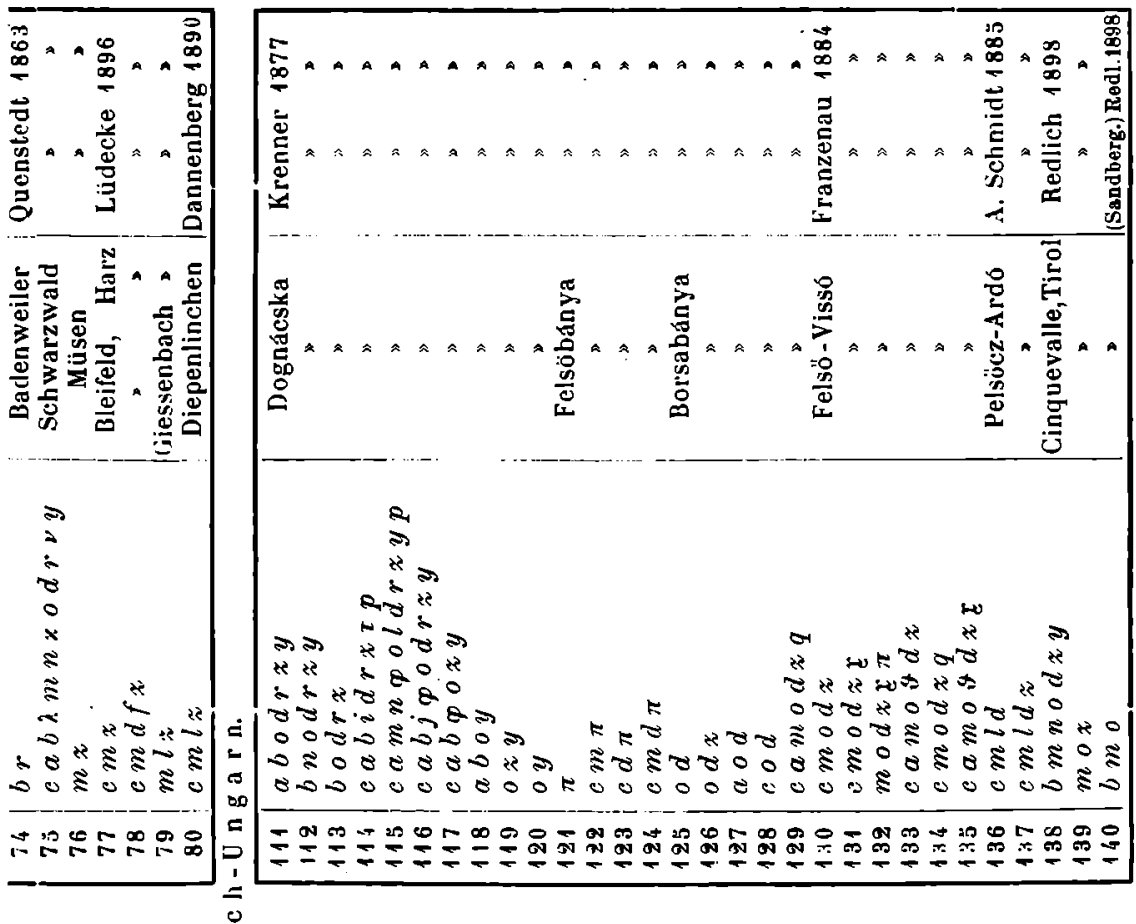

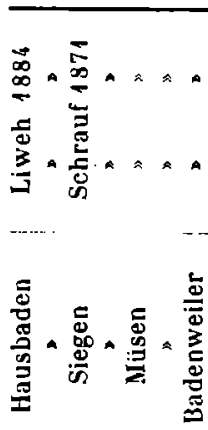

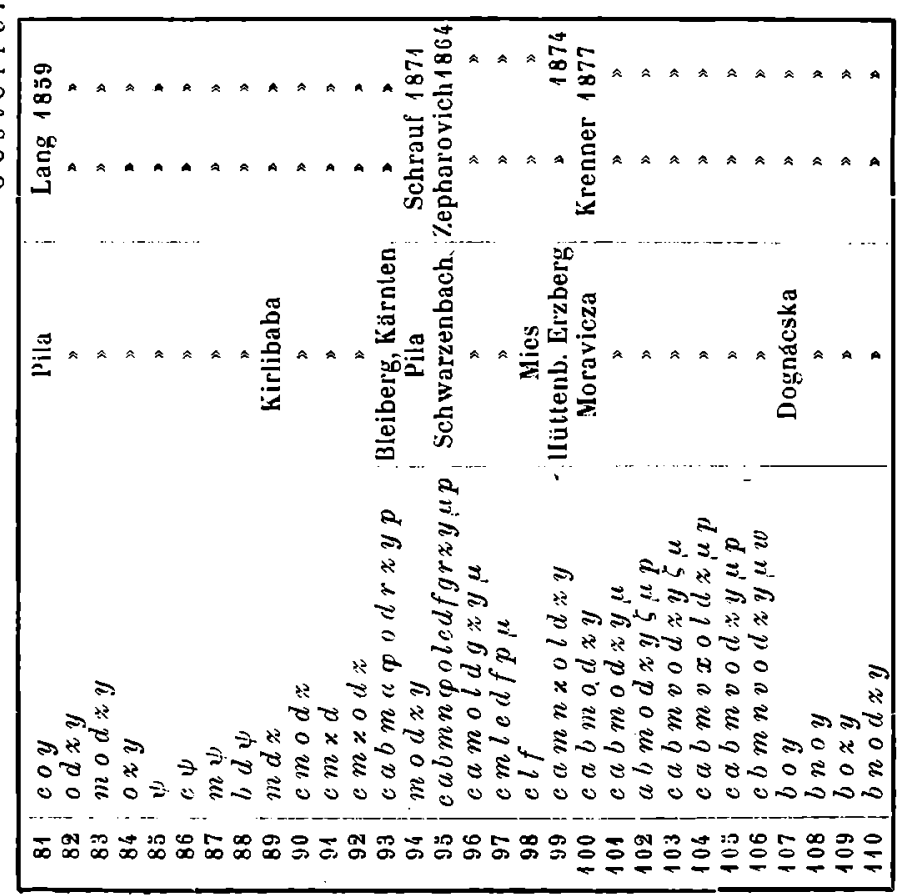




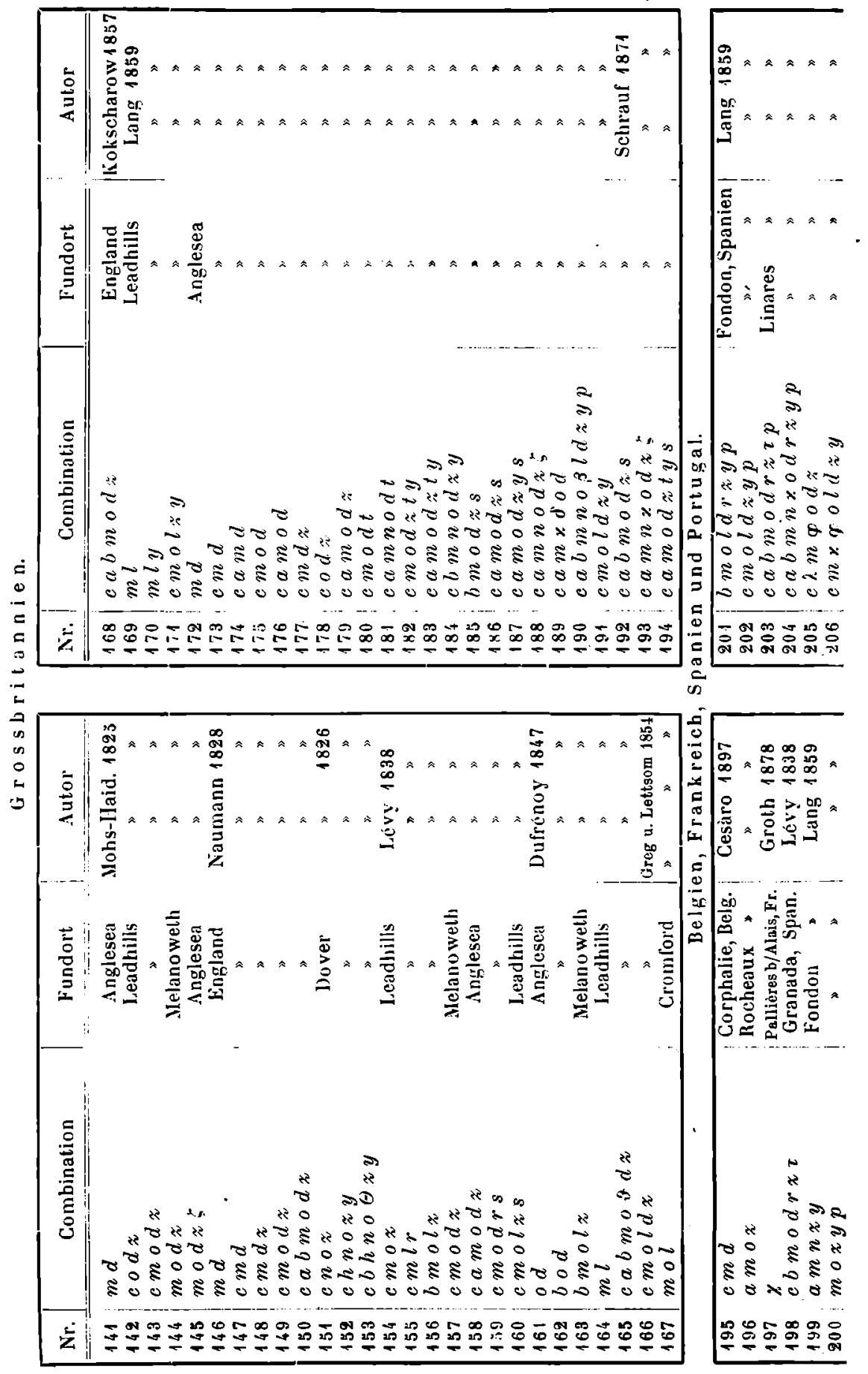


Ueber Anglesit von Monteponi (Sardinien).

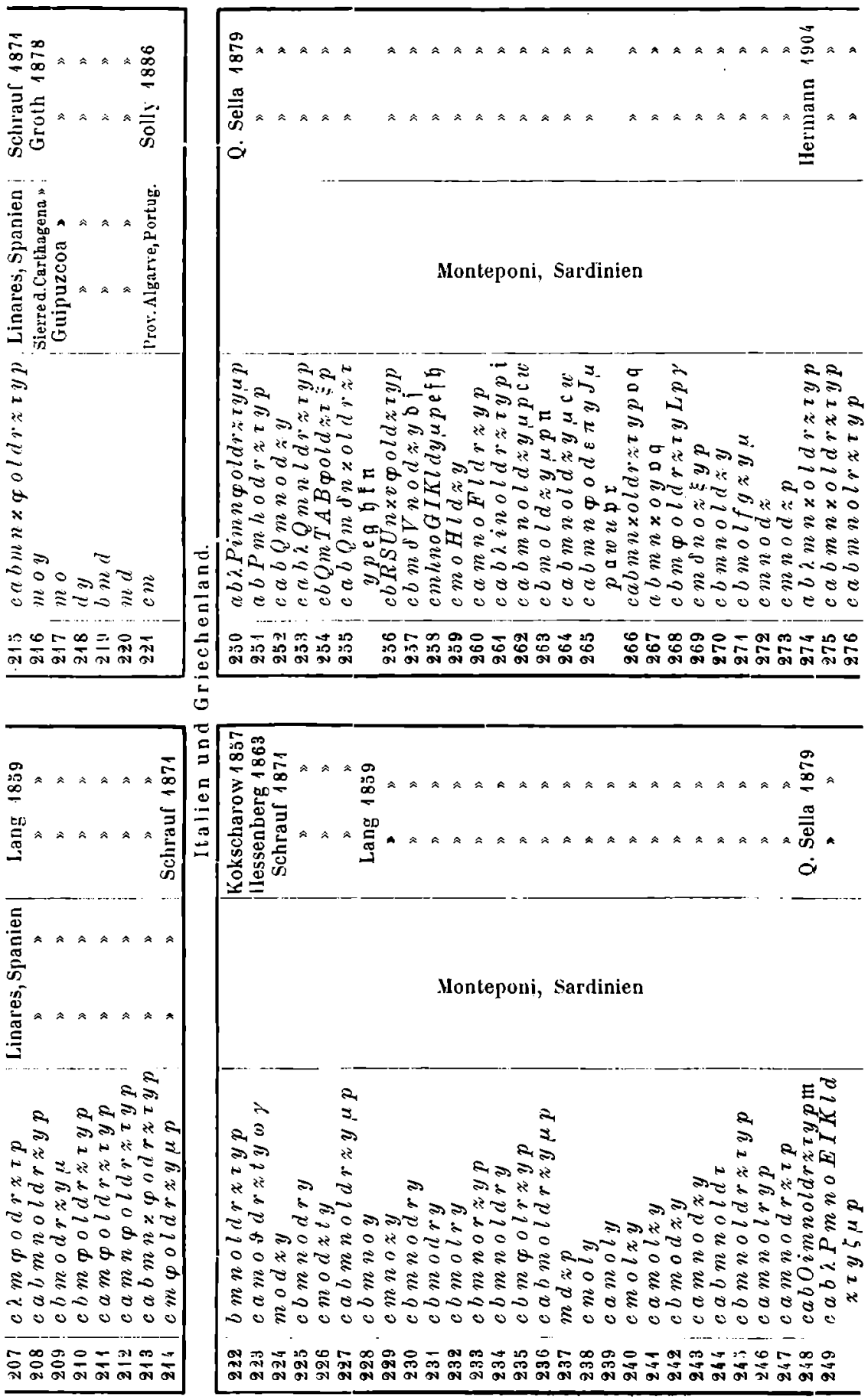

liroth, Zeitschrift f. Krystallogr. XXXIX. 

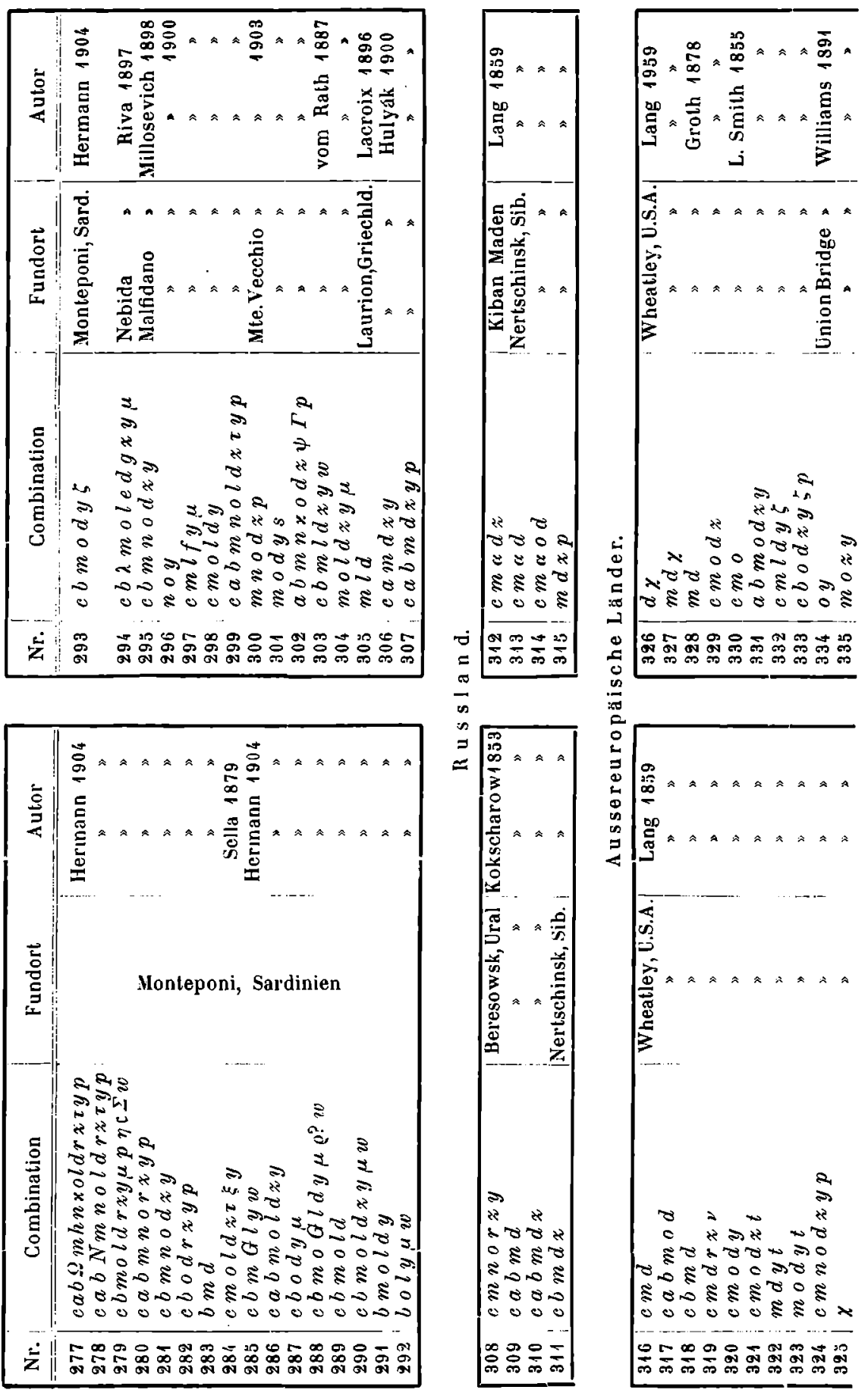
Ueber Anglesit von Monteponi (Sardinien).

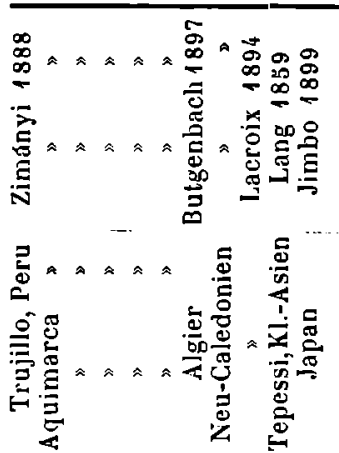

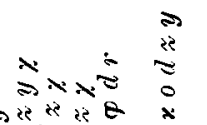

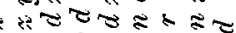

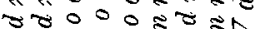

o 0 ह 2 ล

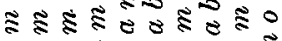

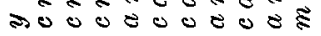

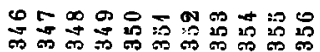

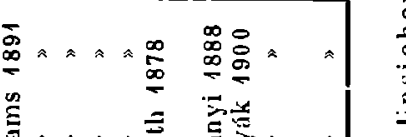

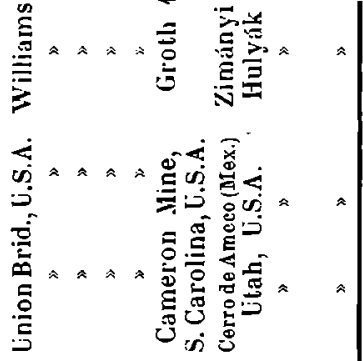

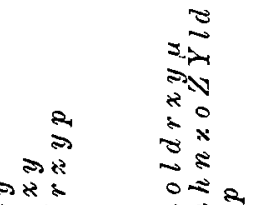

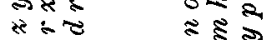

गण 0 स से रुण

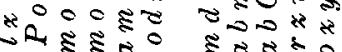

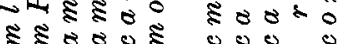

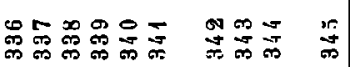

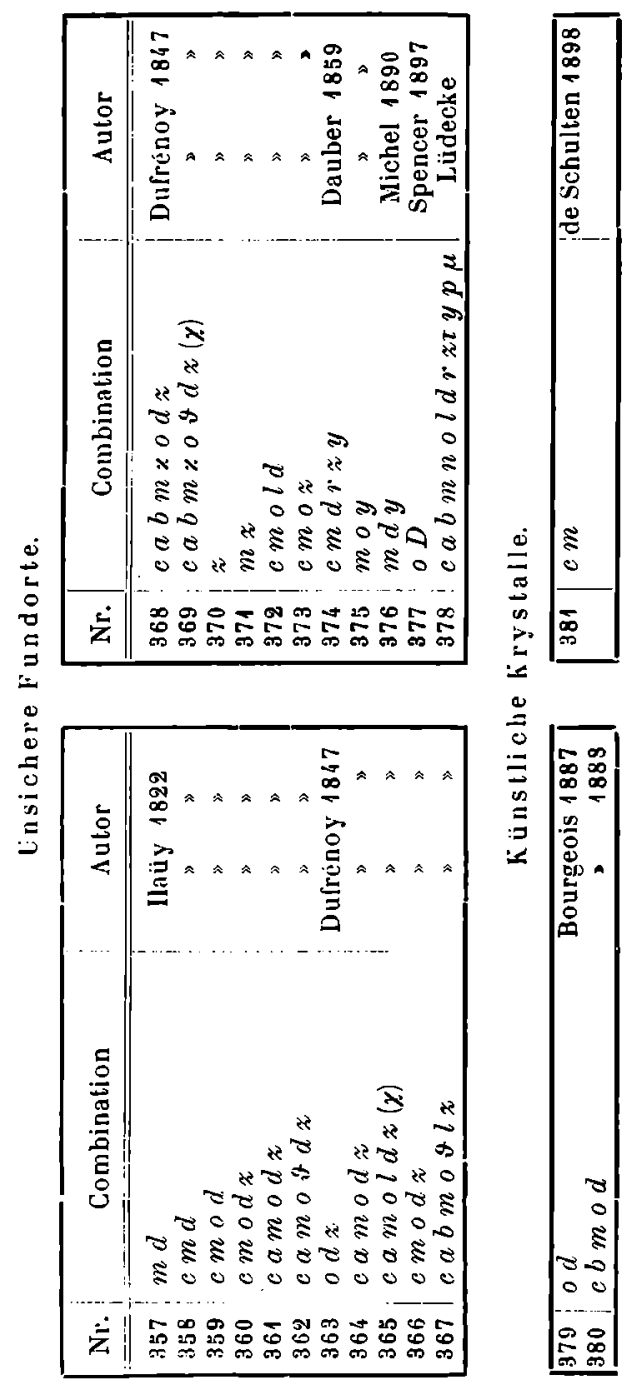




\section{Formen des Anglesits}

mit Angabe des ersten Beobachters und der Häufigkeit.

\begin{tabular}{|c|c|c|c|c|c|c|c|c|c|}
\hline \multirow[b]{2}{*}{ Nr. } & \multirow{2}{*}{$\begin{array}{c}\text { Buchst. } \\
\text { des } \\
\text { Index }\end{array}$} & \multicolumn{3}{|c|}{ Symbole } & \multicolumn{2}{|c|}{ Häufigkeit } & \multirow{2}{*}{$\begin{array}{c}\text { Buchst. } \\
\text { des } \\
\text { Autors }\end{array}$} & \multirow[b]{2}{*}{ Aulor } & \multirow{2}{*}{\begin{tabular}{|c} 
Jahres- \\
zahl
\end{tabular}} \\
\hline & & Gdt. & Miller & Naumann & $\begin{array}{l}\text { Zahl d. } \\
\text { Beob. }\end{array}$ & $\%$ & & & \\
\hline 1 & $e$ & 0 & $\left\{\begin{array}{lll}0 & 0 & 1\end{array}\right\}$ & $0 P$ & 256 & 67,4 & $n$ & Haüy & 1822 \\
\hline 2 & $a$ & $0 \infty$ & $\{010\}$ & $\infty \breve{p} \infty$ & 122 & $32,0^{\circ}$ & $x$ & , & , \\
\hline 3 & $b$ & $\infty 0$ & $\{100\}$ & $\infty \bar{l} \infty$ & 144 & $37,8 \cdot$ & $o$ & - & 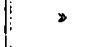 \\
\hline 4 & $M$ & $4 \infty$ & $\{410\}$ & $\infty \ddot{P}_{4}$ & & & & Jeremejew & 1883 \\
\hline 5 & $N$ & $3 \infty$ & $\{310\}$ & $\infty \bar{p} 3$ & 1 & 0,2 & & , & , \\
\hline 6 & $O$ & $5 \infty$ & $\{520\}$ & $\infty \bar{l}_{\xi}^{g}$ & 1 & 0,2 & & Sella & 1879 \\
\hline 7 & $\lambda$ & $2 \infty$ & $\{240\}$ & $\infty \bar{P} q$ & 9 & 2,3 & & Quenstedt & 1855 \\
\hline 8 & $P$ & $7 \infty$ & $\{740\}$ & $\infty \bar{P}_{7}^{7}$ & 4 & $1,0$. & & Sella & 1879 \\
\hline 9 & $i$ & 100 & $\{320\}$ & $\infty \bar{P}_{2}^{4}$ & 4 & $1,0^{\circ}$ & $i$ & Krenner & 1877 \\
\hline 10 & $C$ & $\because \infty$ & $\{10.7 .0\}$ & $\infty \ddot{P} 19$ & 1 & 0,2 . & $C$ & Hulyák & 1900 \\
\hline 14 & $Q$ & $1 \infty$ & $\{430\}$ & $\infty P_{3}^{7}$ & 4 & $1,0$. & & Sella & 1879 \\
\hline 12 & 2 & 500 & $\{340\}$ & $\infty \bar{P}_{\bar{z}}^{\mathrm{a}}$ & 1 & 0,2 . & $g$ & Hermann & 1904 \\
\hline 13 & $R$ & ${ }_{0}^{10} \infty$ & $\{10.9 .0\}$ & $\infty \bar{P} \mu_{9}^{0}$ & 1 & 0,2 . & & Sella & 1879 \\
\hline 14 & $m$ & $\infty$ & $\{110\}$ & $\infty P$ & 310 & 81,4 & $P$ & Haüy & 1822 \\
\hline 13 & $S$ & $\infty \frac{10}{30}$ & $\{9.10 .0\}$ & $\infty \mathscr{P}^{1} 0$ & 1 & 0,2 & & Sella & 1879 \\
\hline 16 & $T$ & $\infty \frac{8}{7}$ & $\{780\}$ & $\infty \breve{p}$ & 1 & 0,2 . & & * & , \\
\hline 17 & $\bar{U}$ & $\infty$ & $\{790\}$ & $\infty P_{4}^{7}$ & 1 & 0,2 . & & 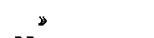 & , \\
\hline 18 & $h$ & $\infty$ & $\{340\}$ & $\infty \breve{P}_{\hat{3}}$ & 6 & $1,5^{\circ}$ & $b$ & Naumann & 1826 \\
\hline 19 & $\delta$ & $\infty_{3}^{3}$ & $\{230\}$ & $\infty \breve{P}_{3: 2}^{3}$ & 4 & $1,0^{\circ}$ & $\delta$ & Lang & 1859 \\
\hline 20 & $V$ & $\infty$ & $\{580\}$ & $\infty P_{i}^{8}$ & 1 & 0,2 . & & Sella & 1879 \\
\hline 21 & $n$ & $\infty 2$ & $\{120\}$ & $\infty \breve{P}_{2}^{3}$ & 94 & 24,7 & $c$ & Naumann & 1826 \\
\hline 22 & $x$ & $\infty 3$ & $\{130\}$ & $\infty \breve{P}_{3}$ & 24 & $6,3$. & & Phillips & 1818 \\
\hline 23 & $W$ & $\infty$ & $\{270\}$ & $\infty \mathscr{P}_{7}$ & 1 & 0,2 . & $\varepsilon$ & Lang & 1859 \\
\hline 24 & $A$ & $0_{16}^{1}$ & $\{0.1 .16\}$ & ${ }_{16}^{1} \breve{p}$ & 1 & 0,2 & & Sella & 1879 \\
\hline 25 & $\alpha$ & $0 \frac{1}{8}$ & $\{018\}$ & $\frac{1}{M} \breve{P} \infty$ & 4 & $1,0^{\circ}$ & $x$ & Lang & 1859 \\
\hline 26 & $j$ & $0 \frac{2}{11}$ & $\{0.2 .11\}$ & $\frac{2}{1}+\breve{p} \infty$ & 1 & 0,2 . & $j$ & Krenner & 1877 \\
\hline 27 & $\ddot{B}$ & 0.2 & $\{029\}$ & 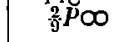 & 1 & 0,2 & & Sella & 1879 \\
\hline 28 & $v$ & $0 . \frac{1}{3}$ & $\{013\}$ & $\breve{p}_{\infty}$ & 5 & 1,3 . & $v$ & Krenner & 1877 \\
\hline 29 & $\varphi$ & $0 \frac{1}{2}$ & $\{012\}$ & 1 & 22 & i, 8 . & $\varphi$ & Lang & 1859 \\
\hline 30 & $x$ & $0 \frac{3}{3}$ & $\{033\}$ & ${ }_{7}^{2}$ & 1 & 0,2 . & $x$ & Krenner & 1877 \\
\hline 31 & $o$ & 01 & $\{011\}$ & $\breve{P}_{\infty}$ & 276 & 72,5 & $t$ & Haüy & 1822 \\
\hline $3 z$ & $\vartheta$ & 02 & $\{021\}$ & $2 \breve{P} \infty$ & 7 & $1,8$. & & Phillips & 1818 \\
\hline 33 & $\beta$ & 03 & $\{031\}$ & $3 \breve{P}_{\infty}$ & 1 & 0,2 . & $\beta$ & Lang & 1859 \\
\hline 34 & $k$ & $: \frac{1}{1}=0$ & $\{1.0 .24\}$ & ${ }_{2}^{1} \bar{P} \infty$ & 1 & 0,2 . & $k$ & Liwelı & 1884 \\
\hline 35 & $B$ & 20 & $\{1.0 .22\}$ & $1 \bar{P} \infty$ & 1 & 0,2 . & & Sella & 1879 \\
\hline 36 & $Z$ & $\frac{1}{2} 0$ & $\{1.0 .16\}$ & $\frac{1}{1} P \infty$ & 1 & 0,2 . & $D$ & Hulyák & 1900 \\
\hline 37 & $F$ & $\frac{1}{15} 0$. & $\{1.0 .15\}$ & $-\frac{1}{r_{5}} \cdot \bar{p} \infty$ & 1 & 0,2 . & & Sella & 1879 \\
\hline 38 & $G$ & $\frac{1}{8} 0$ & $\{108\}$ & $\frac{1}{x} \infty$ & 3 & 0,8 & & - & $\bullet$ \\
\hline 39 & $H$ & $\frac{5}{15} 0$ & $\{2.0 .15\}$ & $\frac{\overline{2}}{15}, \bar{P} \infty$ & 1 & 0,2 . & & 3 & : \\
\hline
\end{tabular}




\begin{tabular}{|c|c|c|c|c|c|c|c|c|c|}
\hline & Bucbst. & & Symbole & & Häufig & keit & Buchst.i| & & \\
\hline Nr. & $\begin{array}{c}\text { des } \\
\text { Index }\end{array}$ & Gdt. & Miller & Naumann & $\begin{array}{l}\text { Zahl d. } \\
\text { Beob. }\end{array}$ & $\%$ & $\begin{array}{c}\text { des } \\
\text { Autors }\end{array}$ & Autor & $\begin{array}{c}\text { Jahres- } \\
\text { zahl }\end{array}$ \\
\hline $\begin{array}{l}40 \\
41 \\
42\end{array}$ & $\frac{J}{K}$ & $\begin{array}{l}\frac{5}{7} 0 \\
\frac{1}{6} 0 \\
2 \\
20\end{array}$ & $\begin{array}{l}\{107\} \\
106\}\end{array}$ & $\begin{array}{l}\frac{1}{7} \bar{P} \infty \\
1 \bar{P} \\
\overline{2} \\
\bar{p}\end{array}$ & $\begin{array}{l}2 \\
2 \\
1\end{array}$ & $\begin{array}{l}0,5 \\
0,5 \\
0.9 .\end{array}$ & & Sella & 1879 \\
\hline 42 & $Y$ & $\frac{2}{9} 0$ & $\{209\}$ & $\frac{\bar{z}}{9} \bar{P} \infty$ & 1 & 0,2 & $Y$ & Hulyák & 1900 \\
\hline 43 & $l$ & 40 & $\{101\}$ & ${ }_{4} \bar{P} \infty$ & 105 & 27,5 & & Mohs & 1824 \\
\hline 44 & $e$ & $\frac{10}{30}$ & $\{103\}$ & $\bar{p}$ & 3 & 0,8 & & Zepharovich & 18.2 \\
\hline $4: 5$ & $d$ & $\frac{1}{2} 0$ & $\{102\}$ & $\frac{1}{2} \bar{P} \infty$ & 277 & $72,7^{\circ}$ & $P$ & Haüy & 1824 \\
\hline 46 & $\Theta$ & $\frac{1}{6}$ & $\{116\}$ & $\frac{1}{d} P$ & 2 & 0,5 & $d$ & Naumann & 1826 \\
\hline 47 & $f$ & $\frac{1}{4}$ & $\{114\}$ & $P$ & 6 & 1,5 & & Zepbarovich & 1866 \\
\hline 48 & $g$ & $\frac{\pi}{3}$ & $\{113\}$ & $\frac{1}{3} P$ & 3 & 1,3 & & , & - \\
\hline 49 & $r$ & $\frac{1}{2}$ & $\{112\}$ & ${ }_{1}^{1} P$ & 80 & 21,0 & $r$ & Naumann & 1826 \\
\hline $\mathbf{5 0}$ & $\approx$ & 1 & $\{111\}$ & $P$ & $2 / 16$ & 64,6 & $s$ & Haüy & $18 \div 2$ \\
\hline 51 & $\boldsymbol{\tau}$ & 2 & $\{221\}$ & $2 P$ & 41 & 17,5 & & Kayser & 1834 \\
\hline $3 z$ & $\xi$ & 3 & $\{331\}$ & $3 P$ & $\mathbf{3}$ & 0,8 & & Sella & 1879 \\
\hline 53 & $\nu$ & 11 & $\{212\}$ & $\bar{p} g$ & 3 & 0,8 & & Quenstedt & $185 \%$ \\
\hline 34 & $t$ & 12 & $\{121\}$ & $2 \breve{P} 2$ & 15 & 3,9 & & Mohs & 1824 \\
\hline $\mathbf{5 s}$ & $\varepsilon$ & 13 & $\{131\}$ & $3 \breve{P 3}$ & 1 & 0,2 . & & Sella & 1899 \\
\hline 56 & r & $T^{\frac{1}{2}-1}$ & $\{1.12 .12\}$ & $\breve{p}_{4} z$ & $\mathbf{3}$ & 0,8 & $k$ & Franzenau & 1884 \\
\hline 57 & $q$ & $\frac{1}{61}$ & $\{166\}$ & $\breve{p}_{i j}$ & 2 & $0, \ddot{3}$ & $q$ & Krenner & 1877 \\
\hline 58 & $\pi$ & $\frac{1}{5}-1$ & $\{155\}$ & $\breve{p}_{5}$ & 6 & $1,5$. & $\pi$ & ' & . \\
\hline $\mathbf{5 9}$ & $\%$ & $\frac{j}{4} 1$ & $\{144\}$ & $\breve{p}_{4}$ & 9 & 2,3 & $y$ & Lang & $18 \vdots 9$ \\
\hline 60 & $\psi$ & $\frac{1}{3} 1$ & $\{1133\}$ & $\vec{p}_{3}$ & 5 & 1,3 & $\psi$ & $>$ & • \\
\hline 61 & $D$ & 21 & $\{255\}$ & $\breve{y}_{5}$ & 1 & 0,2 . & $D$ & Spencer & 1897 \\
\hline 62 & $y$ & 妾1 & $\{122\}$ & $\breve{p}_{z}^{2}$ & 198 & 58,0 & & Phillips & 1818 \\
\hline 63 & $\ell$ & $\frac{2}{3} 1$ & $\{233\}$ & $\breve{p}_{\frac{3}{2}}$ & & & & Jeremejew & 1883 \\
\hline 64 & $\omega$ & $\therefore 7$ & $\{214\}$ & $\bar{p} 2$ & 1 & 0,2 . & & Sella & 1879 \\
\hline 65 & $s$ & 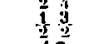 & $\{132\}$ & $\frac{1}{2} y_{3}$ & 11 & 2,9 & & Levy & 1838 \\
\hline 66 & $\zeta$ & $\frac{1}{2}$ & $\{142\}$ & $\mathbf{z} \breve{\mu}_{4}$ & 9 & 2,3 & & Mohs & 1824 \\
\hline 67 & $L$ & 14 & $\{236\}$ & $\frac{1}{42}$ & 1 & 0,2 & & Sella & 1879 \\
\hline 68 & $\gamma$ & $\begin{array}{ll}1 \\
1 \\
3 \\
3 \\
3\end{array}$ & $\{123\}$ & 3 & 2 & 0,5 & $\sigma$ & Kayser & $1 \cdot 34$ \\
\hline 69 & $a$ & $\frac{1}{3}$ & $\{143\}$ & $\frac{1}{3} \breve{p}_{4}$ & 1 & 0,2 & & Sella & 1879 \\
\hline 70 & $J$ & 2) & $\left\{1.10 .20^{\prime}\right.$ & $15 \bar{p}_{10}$ & 1 & 0,2 & & , & , \\
\hline 71 & $\mu$ & 11 & $\{124\}$ & ${ }_{2}^{1} \bar{p}$ & 35 & 9,2 & & Lang & 1859 \\
\hline 72 & $p$ & tit & $\{32\}$, & $\frac{3}{4} \overline{\frac{3}{2}}$ & 90 & 23,6 & $l$ & Haüy & 1822 \\
\hline 73 & e & $\frac{3}{2} 2$ & $\{342\}$ & $2 \breve{P}_{3}$ & $1(2)$ & 0,2 & $\varphi$ & Lang & 1859 \\
\hline 74 & $\eta$ & $\frac{1}{5} \frac{2}{5}$ & $\{125\}$ & $2 \breve{p 2}$ & 1 & 0,2 . & $\eta$ & Hermann & 1904 \\
\hline 75 & $\stackrel{T}{\Gamma}$ & $\frac{5}{5} \frac{5}{5}$ & $\{135\}$ & $\frac{5}{5} \breve{p}_{3}$ & 1 & 0,2 . & 7 & Millosevich & 1903 \\
\hline 76 & $\mathfrak{b}$ & $T^{1} \frac{1}{1}+\frac{1}{3}$ & $\{1.11 .13\}$ & $1, \breve{p}_{11}$ & 1 & 0,2 & & Sella & 1879 \\
\hline 77 & c & $\frac{1}{16}$ & $\{126\}$ & $\frac{142}{3} 2$ & 3 & 0,8 & & , & • \\
\hline 78 & $u$ & 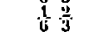 & $\{146\}$ & 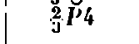 & 2 & 0,5 & & , & - \\
\hline 79 & y & t $\frac{2}{7}$ & $\{127\}$ & $\stackrel{2}{\mu}$ & 1 & 0,2 . & & Hermann & 1904 \\
\hline 80 & $w$ & $\frac{1}{8} \frac{1}{4}$ & $\{128\}$ & 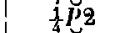 & 10 & 2,6 & & Krenner & 1877 \\
\hline 81 & $D$ & $\frac{5}{2} 3$ & $\{56 \geq\}$ & $3 \overrightarrow{P G}$ & 1 & 0,2 & & Sella & 1879 \\
\hline
\end{tabular}




\begin{tabular}{|c|c|c|c|c|c|c|c|c|c|}
\hline \multirow[b]{2}{*}{$\mathrm{Nr}$. } & \multirow{2}{*}{$\begin{array}{l}\text { Buchst. } \\
\text { des } \\
\text { Index }\end{array}$} & \multicolumn{3}{|c|}{ Symbole } & \multicolumn{2}{|c|}{ Häuligkeit } & \multirow{2}{*}{$\begin{array}{l}\text { Buchsist. } \\
\text { des } \\
\text { Autors }\end{array}$} & \multirow[b]{2}{*}{ Autor } & \multirow{2}{*}{$\begin{array}{c}\text { Jahres- } \\
\text { zabl }\end{array}$} \\
\hline & & Gdt. & Miller & Naumann & $\begin{array}{r}\text { Zahl d. } \\
\text { Beob. }\end{array}$ & $\%$ & & & \\
\hline 82 & $\mathfrak{c}$ & $4 \%$ & $\{892\}$ & $\frac{8}{2} \breve{p} 9$ & 2 & 0,5 & & Sella & 1879 \\
\hline 83 & $\mathfrak{f}$ & $\frac{7}{2} 4$ & $\{782\}$ & $4 \mathrm{PG}$ & 1 & 0,2 & & 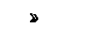 & , \\
\hline 84 & $\mathfrak{g}$ & 54 & $\{10.11 .2\}$ & $\frac{11}{2} P \frac{11}{10}$ & 1 & 0,2 & & > & , \\
\hline 85 & $\mathfrak{h}$ & 56 & $\{301\}$ & $-6 \breve{p}_{0}^{0}$ & 2 & 0,5 & & ๖ & - \\
\hline 86 & $\mathrm{i}$ & $\frac{9}{25}$ & $\{9.10 .2\}$ & 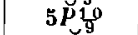 & 1 & 0,2 & & - & $\bowtie$ \\
\hline 87 & $\mathfrak{t}$ & 67 & $\{671\}$ & $7 \breve{P}_{6}^{8}$ & 1 & 0,2 . & & > & - \\
\hline 88 & $\mathfrak{n} \mathbf{t}$ & $13^{16}$ & $\{11.12 .2\}$ & $6 \breve{p}_{12}$ & 1 & $0,2^{\circ}$ & & , & $\$$ \\
\hline 89 & $\mathfrak{n}$ & 78 & $\{781\}$ & $8 \breve{P}_{4}^{19}$ & 1 & 0,2 . & & s & . \\
\hline 90 & 0 & $7 \cdot 10$ & $\{7.10 .1\}$ & $16 P_{7}^{0}$ & 2 & 0,5 & & , & , \\
\hline 91 & $p$ & $\frac{1}{8} \frac{3}{4}$ & $\{168\}$ & $3 \check{P}_{0}$ & 1 & 0,2 . & & $\Rightarrow$ & " \\
\hline 92 & $\mathfrak{q}$ & $8 \cdot 1^{8}$ & $\{8.10 .1\}$ & $10 \breve{P}_{5}^{5}$ & 2 & 0,5 & & , & , \\
\hline 93 & $\mathbf{t}$ & $4: \frac{3}{5}$ & $\{435\}$ & $s_{3} \bar{P}_{3}^{4}$ & 1 & 0,2 . & $\varrho$ & Liweh & 1894 \\
\hline 94 & $\mathfrak{r}$ & $\frac{2}{5} 9$ & $\{995\}$ & $\breve{p q}$ & 1 & 0,2 . & & Sella & 187.9 \\
\hline 9.5 & i & $\frac{1}{2} \frac{y}{2}$ & $\{792\}$ & $9 \vec{p} \frac{9}{4}$ & 1 & 0,2 . & & D & - \\
\hline
\end{tabular}

Die voranstehenden Statistiken sollten, wie einleitend bemerkt wurde, dazu dienen:

1) zu ermüglichen, für jede Form den Ort und die Art der Beobachtung, den Autor und den Publicationsort zu finden;

2) zu zeigen, mit welchen Formen jede Einzelform in Combination trill.

3) Die Zahl der Combinationen zu bestimmen, in der jede Form gefunden wurde. Hierdurch erhalten wir ein Maass für die relative Häufigkeit der Formen und zugleich eine Rangordnung, welche wir mit derjenigen vergleichen können, die aus der Gesammtheit der Formen im Projectionsbilde und dem Verlauf der krummen Flächen sich entnehmen lässt.

Bezeichnen wir als Hüufigkeit einer Form die Zahl von Combinationen, in denen sie auftritt, so können wir diese direct in der Zahl der Beobachtungen ausdrücken, oder auch in Procenten der überhaupt beobachteten Combinationen.

In der nachstehenden Uebersicht sind die Formen der 381 bekannten Combinationen ihrem lfäufigkeitsrange nach geordnet.

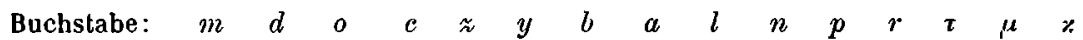

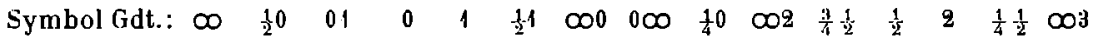

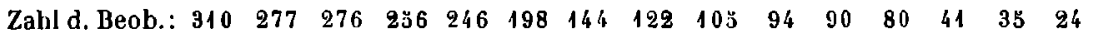
Hłufigkeit $\%: 81,4 \quad 72,7 \cdot 72,5 \quad 67,4 \quad 64,6 \quad 52,0 \quad 37,8 \quad 32,0 \cdot 27,5 \cdot 24,7 \quad 23,6 \quad 21,0 \quad 17,5 \quad 9,2 \quad 6,3 \cdot$

\begin{tabular}{|c|c|c|c|c|c|c|c|c|c|c|c|c|c|c|c|}
\hline Buchstabe: & & $t$ & 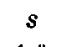 & $w$ & $\lambda$ & $\zeta$ & $x$ & it & $h$ & $f$ & $\pi$ & $v$ & $g$ & $\psi$ & $P$ \\
\hline Symbol & $0 \frac{1}{2}$ & 12 & $\frac{1}{2} \frac{1}{2}$ & $\frac{1}{8} \frac{1}{4}$ & 200 & 12 & $\frac{1}{4} 1$ & 02 & $\infty \frac{1}{3}$ & $\frac{1}{4}$ & $y$ & $0 \frac{1}{3}$ & 1 & $3^{14}$ & $7 \infty$ \\
\hline hl & 2 & 15 & 11 & 10 & 9 & 9 & 9 & 7 & 6 & 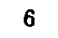 & $f$ & 5 & & 5 & \\
\hline ăufigk & 5, & 3,9 & 2, & 2,6 & $2, ;$ & 2,3 & 2 , & $1,8^{\circ}$ & $1,5$. & 1,5 & $4,5^{\circ}$ & 1,3 & 1,3 & 1,3 &, 0 \\
\hline
\end{tabular}


$\begin{array}{llllllllllllllll}\text { Buchstabe: } & i & Q & \delta & \alpha & P & e & \xi & \nu & \mathcal{E} & \mathfrak{c} & J & K & \Theta & q & \gamma\end{array}$

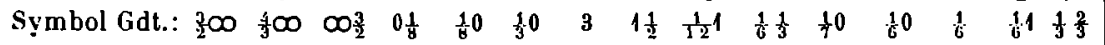
$\begin{array}{llllllllllllllll}\text { Zahl d. Beob.: } & 4 & 4 & 4 & 4 & 3 & 3 & 3 & 3 & 3 & 3 & 2 & 2 & 2 & 2 & 2\end{array}$

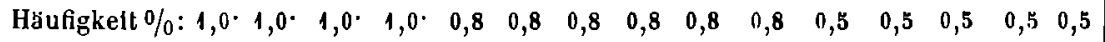

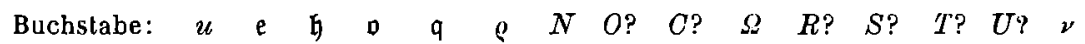

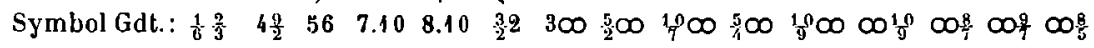

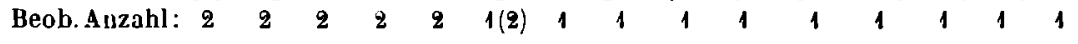

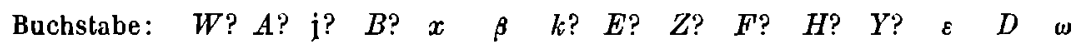

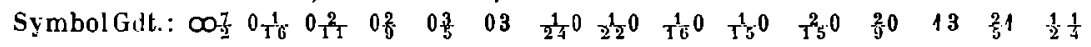

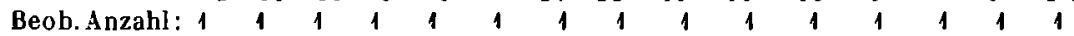

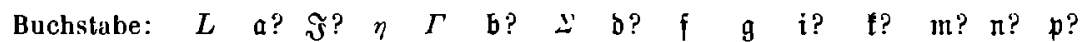

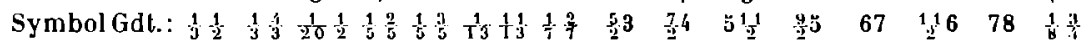

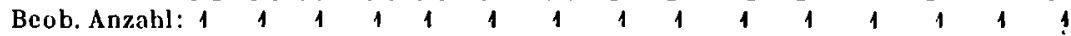

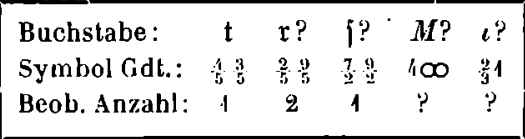

Die mit ? bezeichneten Formen sind aus Gründen, die im Nachstehenden niedergelegt wurden, als unsichere, noch zu bestätigende Flächenarten angesehen worden. Die Formen $M$ und $\iota$ wurden nur an Pseudomorphosen bis jetzt beobachtet.

\section{Unsichere Formen.}

Die Form $d$ ist nach Angabe von Prof. Goldschmidl aus dem Formenverzeichniss zu lüschen. Derselbe teilt mir darüber Folgendes mil:

"Die Form wurde von mir (diese Zeitschr. 1891, 18, 282) vermuthungsweise aufgestellt und mit $p$ bezeichnet. Sie ergab sich durch Umrechnung der Symbole eines Krystalles, den Lüdecke als Datolith aus dem Wäschgrund angesehen hatte. Der von Lüdecke gemessene Winkel zur Basis betrug aber $76^{0} 33^{\prime}$, derselbe würde der bekannten Form $\tau=2(221)$ entsprechen, bei welcher dieser Winkel $7^{0} 32^{\prime}$ beträgt.

Demnach haben wir also die Combination: $c$ a $b$ m noldr $r z x$ y $p \mu$; der Fundort ist unbekannt.

Die Form $A=4(\$ 1)$ ist von Dana in seinem System of Min. 1892, S. 908 mit ? aufgenommen; ebenso von Goldschmidt in den Winkeltabellen (S. 43). An beiden Stellen ist sie zu streichen.

Die Formen $M=4 \infty(410), N=3 \infty$ (310) und $i=\frac{21}{3}$ (233) wurden von Jeremejew (Verh. Petersb. Min. Ges. 1883; diese Zeitschr. 1883, 
7, 63i) an Pseudomorphosen bestimmt. Da ich $N$ an einem Krystalle von Monteponi (Kryst. 5) nachwies, so fällt diese Form aus der Reihe der unsicheren aus; dagegen bedürfen $M$ und $\iota$, die sonst gut in die Formenreihen des Anglesits passen, noch der Bestätigung und sind bis dahin als fraglich zu bezeichnen.

E. S. Dana 1) giebt nachstehende, von Sella angeführte Formen mit folgenden Worten:

s Sella adds the following observed once only and needing confirmation *

als unsicher an:

$$
\begin{aligned}
& O=\frac{5}{2}(520) ; R=\frac{10}{9} \infty(10.9 .0) ; S=\infty \frac{10}{9}(9.10 .0) ; E=\frac{1}{22} 0(1.0 .22) ; \\
& F=\frac{1}{15} 0(1.0 .15) ; \quad G=\frac{1}{8} 0(108) ; \quad H=\frac{2}{13} 0(2.0 .15) ; \quad A=0 \frac{1}{16}(0.1 .16) ; \\
& \mathrm{m}={ }_{2}^{11} 6 \text { (11.12.2); } \mathrm{n}=7.8 \text { (7.81); } \mathrm{g}=5 \mathrm{5} \frac{1}{2}(10.11 .2) ; \mathrm{i}=\frac{9}{2} 5(9.10 .2) ; \\
& \mathfrak{f}=\frac{7}{2} 4(782) ; \mathfrak{l}=67(671) ; \delta=\frac{5}{2} 3(562) ; \mathfrak{f}=\frac{7}{2} \frac{9}{2}(792) ; \mathfrak{L}=\frac{1}{3} \frac{1}{2}(236) ; \\
& \mathfrak{c}=\frac{1}{6} \frac{1}{3}(126) ; \varepsilon=13(131) ; u=\frac{1}{6} \frac{1}{3}(146) ; \mathfrak{a}=\frac{1}{3} \frac{4}{3}(143) ; \mathfrak{r}=\frac{2}{5}(255) ; \\
& \mathfrak{p}=\frac{1}{8} 3(168) ; \mathfrak{s}=\frac{1}{20} \frac{1}{2}(1.10 .20) ; \mathfrak{b}=\frac{1}{13} \frac{11}{13}(1.11 .13) \text {. }
\end{aligned}
$$

Wenn man Formen als zweifelhaft bezeichnen zu müssen glaubt, die von einem so hervorragenden Beobachter, wie Q. Sella, gegeben sind, so muss dazu ein besonderer Grund vorliegen. Dieser ist durch Sella's eigene Ansicht gegeben (S. 958):

"Il Sella dichiara che parecchi di questi simboli, e per lo sviluppo, e per la natura della forme a cui si riferiscono, non debbono ritenersi per definitivamente assicurati...

Egli nota intanto che molte delle nuove forme costituiscono gruppi di faccie fra loro vicine e parallele a spigoli importanti, i quali sono talvolta rotondati, ripetendo così l'esempio di cristalli a faccie e spigoli in talune direzioni perfetti, ed in altre imperfetti. «

Da nun in Sella's Publication nähere Angaben über die Ausbildung der einzelnen Flächen fehlen, so erscheint es richtig, mit Dana nur diejenigen von Sella's neuen Formen als gesichert anzusehen, die er als wiederholt beobachtet angiebt.

Von den durch Dana als zweifelhaft angesehenen Formen Sella's wurde $u=\frac{1}{6} \cdot \frac{2}{3}(146)$ von Liweh (1884) wiedergefunden und als neu angesehen. Obwohl Liweh's Bestimmung aus dem Zonenverbande an einer gestreiften Fläche gemacht wurde, dient sie doch zur Bestätigung von Sella's Form, die er nicht kannte. Zur weiteren Bestätigung der Form $u$ dient die Wahrscheinlichkeit aus der Discussion der Zahlenreihen (cf. unten S. 31 und 34). Danach dürfte $u$ als gesichert anzusehen sein.

Wie oben beschrieben, war ich in der Lage, Sella's $G=\frac{1}{2} 0$ (108) durch Beobachtung an zwei Krystallen von Monteponi (Kryst. 11 und 14)

1) System 1892, 908. 
und $\mathfrak{c}=\frac{1}{6} \frac{1}{3}(126)$ an einem Krystalle von demselben Fundorte (Kryst. 6) zu bestätigen. Beide Formen passen ebenfalls in dic Zahlenreihen, wie die unten stehende Discussion zeigt. Sie sind mithin aus dem Verzeichniss der unsicheren Formen auszuscheiden.

Dagegen müchte ich aus denselben Gründen, die Dana anführt, die Formen $T=\infty \frac{8}{7}(\mathbf{7 8 0}), U=\infty \frac{9}{7}(790), V=\infty_{\frac{8}{8}}^{8}(580)$ und $\left.B=0.2 .929\right)$ von Sella als noch zu bestätigende anführen.

Ferner gehört hierher die Form $\mathfrak{x}=\frac{1}{12} 1$ (12.12.1), welche Franzenau an drei Krystallen beobachtete und $k$ nannte. Er giebt Folgendes an (Term. Füz. 1884, 8, 121):

Kryst. $2 \ldots$ (12.12.1) tritt als schmaler Streifen zwischen (111) und (110) auf. An diesem Krystalle ist die neue Fläche verhälnnissmässig am besten ausgebildet. .

Eine weitere $\Lambda$ uskunft über ihre Flïchenbeschaffenheit ist aber nicht zu finden, auch kann man keine Schlüsse aus den idealisirten Bildern über die Ausbildung und das Auftreten dieser Form ziehen. Ihr hochzahliges Symbol, sowie ihr auffallendes Benehmen in der Zahlenreihe lassen auf eine Vicinale schliessen.

Die Form $\alpha=0 \frac{1}{8}(018)$ wurde von Lang (S. 278) an drei Krystallen von Kiban Maden gefunden. Er sagt darüber Folgendes:

- Zur Bestimmung des Symbols der Fläche $\alpha$ wurden ferner gemessen (Fig. 67) $(018)(001)=8^{0} 50^{\prime}$ ca. $\left(9^{0} 9^{\prime}\right) ;(018)(102)=40^{0}$ ca. $\left(40^{0} 16^{\prime}\right)$; $(018)(110)=84^{\circ} 7^{\prime}\left(84^{0} 22^{\prime}\right)$; alle diese Werthe sind nur mit geringer Genauigkeit zu messen, da die Fläche 018 immer rauh ist. \&

Diese Form passt nur schlecht in die Zahlenreihen des Anglesits, so dass die Vermuthung nahe liegt, dass es sich ebenfalls um eine Vicinale handelt, bez. dass dieser Form ein anderes Symbol zukommt.

$W=\infty 7(270)$ wurde von Lang $\left.{ }^{1}\right)$ gefunden, und zwar an einem Krystalle von Maden Tepessi. Der Autor giebt keine nähere Beschreibung dieser Form, nur auf S. 936 findet sich die $\Lambda$ ngabe $\approx 270$ rauh». Das Symbol passt wenig in die Zahlenreihe, so dass sie bis zu ihrer Bestitigung als unsicher zu bezeichnen sein dürfte.

Die Form $j=0 \frac{2}{T}(0.2 .11)$ findet sich bei Krenner. Er sagt über dieselbe Folgendes (diese Zeitschr. 1877, 1, 331):

*Die Neigung der neuen Fläche zur horizontalen Endflïche wurde zu (0.2.11) $(001)=13^{0} 20^{\prime}$ gemessen, während berechnet sich $13^{0} 11,7^{\prime}$ ergiebt; (015) (001) hingegen würde $14^{\circ} 7,6^{\prime}$ [richtiger $14^{\circ} 28^{\prime}$ ] verlangen. Ich habe diese Kanten wiederholt gemessen, doch widersprechen dic erzielten Werthe immer den vermutheten einfachen Indices. *

Die Form passt wenig in die Zahlenreihen.

1) Vgl. Lang S.28 . Messung mit Anlegegroniometer: $(270):(\overline{270})=40^{\circ}\left(\right.$ ber. $\left.40^{0} 0^{\prime}\right)$ $(270):(110)=32(-3152)$ 


\section{Discussion der Symbolzahlen.}

Nach Untersuchungen von V. Goldschmidt ist es müglich eine Discussion der Symbolzahlen jeder Zone vorzunehmen, so dass man aus der Reihenfolge der Zahlen Schlüsse auf die Entwickelung der Formen einer solchen Zone ziehen kann. Dabei zeigt es sich, ob ein Zonenstück eine freie Entwickelung hat, die dann dem Gesetze der Complication folgt, ob es in mehrere freie Stücke zerfält, und welches die Endknoten $(0 . \infty)$ und die Dominanten (1) eines jeden freien Stückes sind. Ferner, ob Störungen im Verlaufe des Zonenstückes stattgefunden haben und welches die Rangordnung der einzelnen Flächenarten ist.

Wichtige Knoten machen sich in der Discussion der Zahlen, wie im Projectionsbilde dadurch kenntlich, dass sie die Endknoten wichtiger Zonenstücke sind. Im Projectionsbilde erkennt man sie daran, dass wichtige Zonenlinien sich in ihnen schneiden oder ausstrahlen, und dass sie von freien Hüfen umgeben werden.

Eine Discussion der Zahlenreihen ist stets in Verbindung mit dem Studium des gnomonischen Projectionsbildes durchzuführen. In diesem Bilde erkennt man die Bedeutung der Spaltung einer Zone in freie Stücke, die in der Regel durch das Einschneiden einer anderen Zone bewirkt wird. Hierdurch wird der getroffene Flächenpunkt beeinflusst, verstärkt; er wird zum Endknoten eines kleineren Zonenstückes.

Ist eine Zone nach der von Goldschmidt gegebenen Vorschrift in ihre frcien Stücke gespalten, so zeigen diese die Zahlen, die das Gesetz der Complication fordert. Wir finden die Normalreihen:

$$
\begin{array}{lllllll}
N_{1}=0 & 1 & \infty & & & \\
N_{2}=0 \frac{1}{2} & 1 & 2 & \infty & & & \\
N_{3}=0.1 & \frac{1}{2} & \frac{9}{3} & 1 \frac{13}{2} & 2 & 3 & \infty
\end{array} .
$$

Selten ist ein freies Stück weiter als bis $N_{3}$ differenzirt. Die Zahlenreihe $N_{2}$ ist oft vollständig, $N_{3}$ dagegen meist lückenhaft entwickelt. Findet man nach dem Spalten in die freien Stücke noch complicirtere Zahlen, etwa solche, die Reihen hüherer Complicationen angehüren: z. B. $\frac{1}{1} \frac{3}{4} \frac{1}{5} \frac{2}{5}$ $\frac{3}{5} \frac{4}{5} \ldots$ oder gar $\frac{1}{7} \frac{2}{7} \ldots \frac{1}{1^{3}} \frac{1}{1}^{2} \ldots$, so ist dieses auffallend. Formen, denen solche Zahlen entsprechen, fordern zu einer kritischen Prüfung heraus. Diese Prüfung hat unter Zurückgehen auf die Originalangaben in der Literatur zu geschehen.

Auf Grund solcher Discussionen wurde im Folgenden eine Prüfung der Formen des Anglesits für die Hauptzonen vorgenommen und eine Rangordnung der wichtigsten Formen aufzustellen versucht.

Bei der Discussion der Zahlen ist es gleichgültig, ob man dieselbe mit den Zahlen $p$ oder $q$ des zweizifferigen Symbols $p q$ vornimmt. Ist in 
einer Zone $p$ constant, so wird man $q$ zur Discussion verwenden müssen, ist $q$ constant, so ist $p$ zu nehmen. Für die Prismen, denen das allgemeine Symbol $\frac{p}{q} \infty=\infty \frac{q}{p}$. zukommt, z. B. $\Omega=\frac{5}{4} \infty=\infty \frac{4}{5}$, discutirl man die Zahl $\frac{p}{q}$ oder $\frac{q}{p}$.

Vor Beginn der Discussion der Reihe prüft man die Symbole, ob dieselben einem gemeinschaftlichen Zonenverbande angehören, indem man die Zonengleichung aufstellt und zusieht, ob die Zahlen eines jeden Symbols dieser Gleichung genügen.

Die Zonengleichung ist eine Gleichung I. Grades von der Form:

$$
a p+b q=1 \text { oder } a p+b q=0
$$

$p$ und $q$ sind die Symbolzahlen, $a$ und $b$ sind für die betreffende Zone constant, für verschiedene Zonen aber variabel.

Beispiel: Zonengleichung: $2 p+2 q=1$.

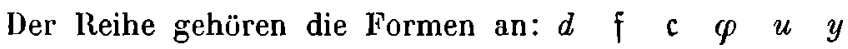

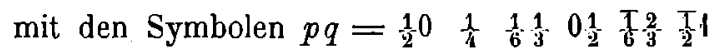
darin ist $q=\begin{array}{llllll}0 & \frac{1}{4} & 1 & \frac{1}{2} & \frac{2}{3} & 1\end{array}$ wir bilden $\frac{v}{1-v}=\begin{array}{lllllll}0 & 1 & 1 & 1 & 2 & 0 & 0\end{array}$

Dic Zahlen sind normal.

Hier, wie überhaupt in der Discussion, bedeutet bei $\frac{v}{v-1} v$ die einzelne Zahl der jeweils vorangehenden Reihe.

1) Prismenzone, seitliches Stück.

$\begin{array}{llllllllllllll}\text { Formen: } & b & M & N & O & \lambda & P & i & C & Q & \Omega & R & m\end{array}$

$\frac{p}{q} \infty=\infty 04 \infty 3 \infty \quad 5 \infty 2 \infty \frac{7}{4} \infty \frac{3}{2} \infty \frac{10}{7} \infty \frac{4}{3} \infty \frac{5}{4} \infty \frac{10}{9} \infty \infty$

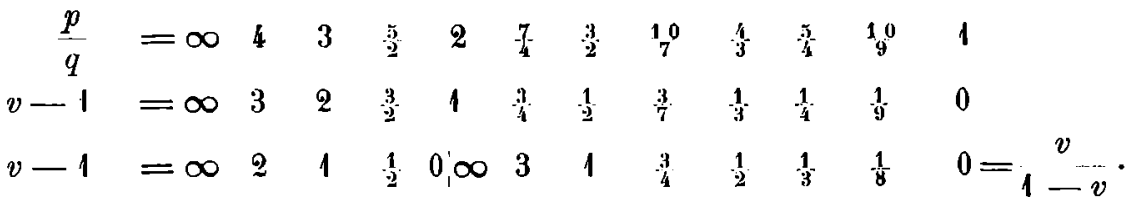

Die Reihe ist bei $\lambda$ zu spalten, dann sind die Zahlen beider freien Stücke bis auf $\frac{3}{4}$ und $\frac{1}{8}$ normal; diese beiden sind hingegen aufallend. $\frac{3}{4}$ entspricht der Form $C={ }_{7}^{10} \infty$ von II ulyák und $\frac{1}{8}$ der unsicheren Form $R={ }_{9}^{10} \infty$ von Sella. Die neue Form $\Omega=5 \infty$ passt gut in die Reihe. 
2) Prismenzone, vorderes Stück.

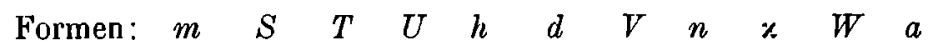

$$
\begin{aligned}
& \frac{q}{p}-\infty=\infty \infty_{9}^{10} \infty_{\frac{8}{7}} \infty_{\frac{9}{7}} \infty_{\frac{4}{3}} \infty_{\frac{3}{2}} \infty_{\frac{8}{5}}^{8} \infty 2 \infty 3 \infty_{\frac{7}{2}} 0 \infty
\end{aligned}
$$

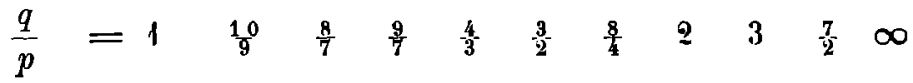

$$
\begin{aligned}
& v-1=0 \quad \frac{1}{9} \quad \frac{1}{7} \quad \frac{2}{7} \quad \frac{1}{3} \quad \frac{1}{2} \quad \frac{3}{5} \quad 1 \quad 2 \quad 2 \quad \frac{5}{2} \quad \infty
\end{aligned}
$$

Nach Ausscheiden der unsicheren Formen $S=\infty \frac{10}{9}, T=\infty \frac{8}{7}$, $U=\infty \frac{9}{7}$ und $V=\infty \frac{8}{5}$ haben wir die Reihe

$$
\begin{array}{ccccccc}
m & h & \delta & n & x & (W) & a \\
0 & 1 & \frac{1}{3} & 1 & 2 & \left(\frac{5}{2}\right) & \infty .
\end{array}
$$

Unter diesen Zahlen ist $\frac{5}{2}$ entsprechend $W=\infty \frac{7}{2}$ auffallend. Da Lang die Messung mit Hülfe des Anlegegoniometers ausgeführt hat, und solche Messungen nur Nährungswerthe ermöglichen, so dürfle die Form nicht als gesichert gelten. (Vgl. vorigen Abschnitt: Unsichere Formen.)

3) Längsdomen. Nach Weglassung der unsicheren Formen $A=0 \frac{1}{16}$, $B=0 \frac{2}{9}$ erhalten wir die Reihe:

$$
\begin{aligned}
& \begin{array}{llllllllll}
c & \alpha & j & v & \phi & x & o & \mathcal{J} & \beta & a
\end{array} \\
& p q=\begin{array}{lllllllllll}
0 & 0 \frac{1}{8} & 0 \frac{2}{11} & 0 \frac{1}{3} & 0 \frac{1}{2} & 0 \frac{3}{5} & 0.1 & 02 & 03 & 0 \infty
\end{array}
\end{aligned}
$$

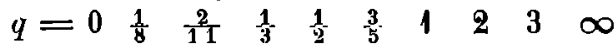

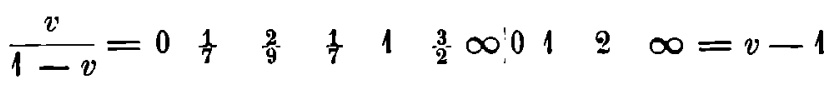

$$
\begin{aligned}
& \frac{3 v}{1-v}=0 \quad \frac{1}{2} \quad \frac{6}{7} \quad 3 \propto 1 \text {. }
\end{aligned}
$$

Die Spaltung bei $\rho$ lässt sich durch das Einschneiden der Zone r $r r$ motiviren, wodurch die Form $a=0 \frac{1}{8}$ an Wahrscheinlichkeit gewinnt. Die Krenner'sche Form $j=0 \frac{2}{1} \mathrm{~T}$ bleibt aber trotzdem mit $\frac{\sigma}{7}$ auffallend und bedarf der Bestätigung. (Im Uebrigen vgl. vorigen Abschnitt: Unsichere Formen.)

4) Querdomen. Nach Ausscheiden der unsicheren Formen $h={ }_{Q_{4}}^{1} 0$; $E=\frac{1}{2} 0, Z=\frac{1}{1} 0, F=\frac{1}{15} 0, H=\frac{2}{15} 0, Y={ }_{9}^{2} 0$ haben wir die Reihe:

$$
\begin{aligned}
& \begin{array}{llllllll}
c & G & J & K & l & e & d & b
\end{array} \\
& p q=0 \quad \frac{1}{8} 0 \quad \begin{array}{lllllll}
7 & \frac{1}{6} 0 & \frac{1}{6} 0 & \frac{1}{3} 0 & 10 & \infty 0
\end{array} \\
& 2 p=0 \quad \frac{1}{4} \quad \frac{2}{7} \quad \frac{1}{3} \quad \frac{1}{2} \quad \frac{2}{3} \quad 1 \quad \infty \\
& \frac{v}{1-v}=0 \quad \frac{1}{3} \quad \frac{2}{5} \quad \frac{1}{2} \quad 1 \quad 2 \quad \infty .
\end{aligned}
$$

$\Lambda$ uffallend ist $\frac{2}{5}$ entsprechend $J==\frac{1}{7} 0$; die Form findet sich nur bei Sella an zwei Krystallen erwähnt. Die übrigen Zahlen sind normal.

5) Pyramidenzone $p=q$. 


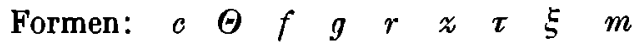

$$
\begin{aligned}
& \begin{array}{lllllllll}
p=0 & \frac{1}{6} & \frac{1}{4} & \frac{1}{3} & \frac{1}{2} & 1 & 2 & 3 & \infty
\end{array} \\
& \frac{v}{1-v}=0 \quad \frac{1}{5} \quad \frac{1}{3} \quad 1 \quad 1 \quad \infty \\
& \frac{2 v}{1-v}=0 \quad 1 \quad 1 \quad 2 \quad \infty=\text { Normalreihe } N_{2} \text {. }
\end{aligned}
$$

6) Zone $q-2 p$.

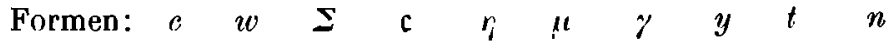

$$
\begin{aligned}
& p q=0 \quad \frac{1}{8}+\frac{1}{7} \frac{2}{7} \quad \frac{1}{6} \frac{1}{3} \quad \frac{1}{5} \frac{2}{5} \quad \frac{1}{4} \frac{1}{2} \quad \frac{1}{3} \frac{2}{3} \quad \frac{1}{2} \quad 12 \quad 02 \\
& q=\begin{array}{llllllllll}
0 & 4 & \frac{1}{7} & \frac{1}{3} & \frac{2}{3} & \frac{1}{2} & \frac{2}{3} & 1 & 2 & \infty
\end{array} \\
& \begin{array}{c}
v \\
T-v
\end{array}=0 \quad 3 \quad \frac{1}{3} \quad \begin{array}{llllll}
3 & 2 & 1 & 2 & \infty
\end{array} \\
& 1-v=0 \quad \frac{1}{2} \quad \frac{2}{3} \quad 1 \quad 2 \quad \infty .
\end{aligned}
$$

Die Zahlen sind normal, wenn wir bei $y$ und $\mu$ spalten. Die neuen Formen $\eta=\frac{1}{3} \frac{2}{3}(125)$ und $\Sigma=\frac{1}{7} \frac{2}{7}$ (127) passen gut in die Reihe.

7) \%one $q=\frac{1}{2}$. Nach Auslassung der unsicheren Form $J=\frac{1}{20} \frac{1}{2}$ haben wir die Reihe:

$$
\begin{array}{ccccccc}
\varphi & \mu & L & r & p & v & b \\
p q=0 \frac{1}{2} & +\frac{1}{2} & 11 \frac{1}{2} & \frac{1}{2} & \frac{3}{4} \frac{1}{2} & 1 \frac{1}{2} & \infty 0 \\
2 p=0 & \frac{1}{2} & \frac{2}{3} & 1 & \frac{3}{2} & 2 & \infty
\end{array}
$$

Die Zahlen sind normal.

8) Zone $q=1$.

$$
\begin{aligned}
& \text { Formen: } \begin{array}{llllllllllll}
o & \mathfrak{x} & q & \boldsymbol{x} & \chi & \psi & D & y & \iota & z & b
\end{array}
\end{aligned}
$$

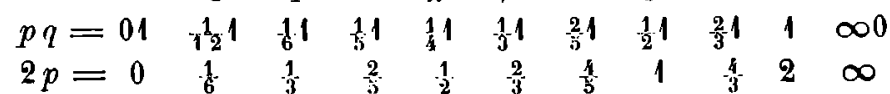

$$
\begin{aligned}
& \frac{v}{1-v}=\begin{array}{llllllll}
0 & \frac{1}{5} & \frac{1}{2} & \frac{2}{3} & 1 & 2 & 4 & \infty
\end{array} \text {. }
\end{aligned}
$$

Zwischen $o$ und $y$ hat sich eine grosse Zahl schwacher Formen entwickelt. Auffallend sind die Zahlen $\frac{4}{3}$ entsprechend $1=\frac{2}{3} 1$, eine von Jeremejew an Pscudomorphosen beobachtete Form, und $\frac{1}{i}$ entsprechend $\mathfrak{x}=\frac{1}{12} 1=k$ (Franzenau). Diese Form dürfte als Vicinale von $o=01$ anzusehen sein.

9) Zone $q=2$.

$$
\begin{array}{rcccccc}
\text { Formen: } \quad \vartheta & \zeta & t & 0 & r & b \\
p q=02 & 12 & 12 & 32 & 2 & \infty 0 \\
p= & 1 & 1 & 3 & 2 & \infty
\end{array}
$$

Die Zahlen sind normal. 
10) Zone $p=\frac{1}{6}$.

$$
\begin{array}{rlccccc}
\text { Formen }: \quad K & \Theta & \mathfrak{c} & u & q & a \\
p q=\frac{1}{6} & \frac{1}{6} & \frac{1}{6} \cdot \frac{1}{3} & \frac{1}{6} \cdot \frac{2}{3} & \frac{1}{6} 1 & 0 \infty \\
3 q= & 0 & \frac{1}{3} & 1 & 2 & 3 & \infty
\end{array}
$$

Die Zahlen sind normal.

11) Zone $p=\frac{1}{4}$.

$$
\begin{array}{rccccl}
\text { Formen: } & l & f & \mu & z & a \\
p q=\frac{1}{4} 0 & \frac{1}{4} & \frac{1}{4} \frac{1}{2} & \frac{1}{4} 1 & 0 \infty \\
2 q= & -\frac{1}{2} & 1 & 2 & \infty=\text { Normalreihe } N_{2} .
\end{array}
$$

12) Zone $p=\frac{1}{3}$.

Formen: $\begin{array}{llllllll}e & g & L & \gamma & \psi & \mathfrak{a} & a\end{array}$

$$
\begin{array}{rlllllll}
p q & =\frac{1}{3} 0 & \frac{1}{3} & \frac{1}{3} \frac{1}{2} & \frac{1}{3} \frac{2}{3} & \frac{11}{3} & \frac{1}{3} \frac{4}{3} & 0 \infty \\
q= & 0 & \frac{1}{3} & \frac{1}{2} & \frac{2}{3} & 1 & \frac{4}{3} & \infty \\
v & & 0 & 1 & 1 & 2 & \infty & =\text { Normalreihe } N_{2} . \\
1-v & & 2 & & & &
\end{array}
$$

Auffallend ist $\frac{1}{3}$. Die Form $\mathfrak{a}=\frac{1}{3} \frac{4}{3}$ bedarf der Bestätigung.

13) Zone $p=\frac{1}{2}$.

$$
\begin{aligned}
& \text { Formen: } d \begin{array}{llllllll}
d & \omega & r & y & s & \zeta & a
\end{array}
\end{aligned}
$$

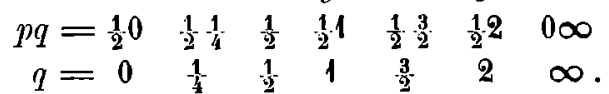

Die Zahlen sind normal, auffaliend ist nur $\frac{1}{4}$ entsprechend $w=\frac{1}{2} \frac{1}{4}$.

14) Zone $2 p+2 q=1$. Vgl. Beispiel S. 491 .

15) Zone $2 p+q=1$. Nach Auslassung der unsicheren Formen $\mathfrak{b}=\frac{1}{\sqrt{3}} \frac{1}{1} \mathfrak{d}$ unp $\mathfrak{p}=\frac{1}{8} \frac{3}{4}$ haben wir die Reihe:

$$
\begin{array}{cccccc}
o & u & \mu & g & d \\
p q=01 & \frac{1}{6} \frac{2}{3} & \frac{1}{4} \frac{1}{2} & \frac{1}{3} & \frac{1}{2} 0 \\
2 p=0 & \frac{1}{3} & \frac{1}{2} & \frac{2}{3} & 1 \\
v & 0 & \frac{1}{2} & 1 & 2 & \infty=\text { Normalreihe } N_{2} . \\
1-v & =0
\end{array}
$$

16) Zone ${ }_{2}^{1} p+\frac{1}{2} q=1$.

Formen: $\begin{array}{lllll}Z & s & \vartheta & \varepsilon & m\end{array}$

$$
\begin{array}{rcccc}
p q=1 & \frac{1}{2} \frac{3}{2} & 02 & 13 & \bar{\infty} \infty \\
q=1 & \frac{3}{2} & 2 & 3 & \infty \\
v-4=0 & \frac{1}{2} & 1 & 2 & \infty=\text { Normalreihe } N_{2} .
\end{array}
$$

17) Zone $-3 p+2 q=1$.

$$
\begin{array}{cccccc}
\text { Formen: } \delta & t & \psi & \varphi & e \\
p q=\infty \frac{3}{2} & 12 & \frac{1}{3} & 0 \frac{1}{2} & \frac{1}{3} 0 \\
q=\infty & 2 & 1 & \frac{1}{2} & 0=\text { Normalreihe } N_{2} .
\end{array}
$$


18) Zone. $q-p=1$. Nach Weglassen der imsietheren Formen $\mathfrak{t}=$ if ifive $67 ;\left\{=\frac{7}{2} \frac{9}{2}\right.$ haben wir die Reihe:

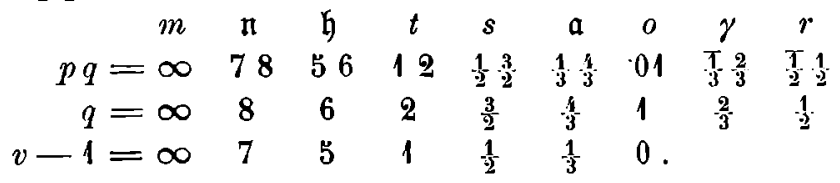

Auffallend sind die Zahlen 7 und 5 entsprechend den Formen $\mathfrak{t}=\mathbf{7 8}$ und $\mathfrak{h}=56$. Die übrigen Zahlen sind normal.

19) Zone. $-2 p+2 q=1$. Nach Auslassen der unsicheren Formen $\mathfrak{n}=\frac{1}{2} 6 ; g=5 \frac{1}{2} ; \mathfrak{i}=\frac{9}{2} \mathfrak{b} ; \mathfrak{f}=\frac{7}{2} 4 ; \mathfrak{d}=\frac{5}{3} 3$ erhalten wir die Reihe:

$$
\begin{aligned}
& \begin{array}{lllllllll}
m & \mathfrak{e} & \varrho & y & u & \rho & \mathfrak{c} & \boldsymbol{f} & d
\end{array} \\
& p q=\infty \quad 4 \frac{9}{2} \quad \frac{3}{2} 2 \quad \frac{1}{2} 1 \quad \frac{1}{6} \frac{2}{3} \quad 0 \frac{1}{2} \quad \frac{7}{6} \frac{1}{3} \quad \frac{T}{4} \frac{1}{4} \quad \frac{T}{2} 0 \\
& q=\infty \quad \frac{9}{2} \quad 2 \quad \begin{array}{lllllll}
2 & 2 & \frac{2}{3} & \frac{1}{2} & \frac{1}{3} & \frac{1}{4} & 0
\end{array} \\
& \infty \quad 2 \quad 1 \quad \frac{1}{3} \quad 0=1 \stackrel{v}{3} .
\end{aligned}
$$

Die Zahlen sind normal bis auf $\frac{9}{2}$, entsprechend $e=4 \frac{9}{2}$.

\section{Projectionsbilder.}

Um zu ersehen, zwischen welchen Punkten die IIauptzonen sich spannen, welche Flächen demnach als Hauptknoten der Entwickelung angesehen werden können, wurden die genannten beobachteten Formen des $\Lambda$ nglesits in gnomonischer Projection aufgezeichnet und zwar in drei Bildern 1), projicirt auf die drei Pinakoide $c=0(001), b=\infty 0(100)$ und $a=$ $0 \infty(010)$.

Die drei Bilder ergänzen sich gegenseitig, indem das, was beim einen Bilde ins Unendliche gerückt ist (Prismen) und sich dadurch der Anschauung entzieht, bei den anderen mitten im Bilde erscheint.

Taf. XIII, Fig. 20 zeigt die stereographische Projection der gesammlen Anglesitformen auf $a=0 \infty(010)$. Dieses Bild hat den Vortheil, dass der Verband mit den Prismenpunkten ersichtlich ist.

Auf allen vier Projectionsbildern wurden die Buchstaben der Formen nur im III. Quadranten eingetragen, um das Bild nicht unklar zu machen. Aufgenommen wurden sichere wie unsichere Formen. Die lieflexe der krummen Flächen wurden roth ${ }^{2)}$ eingetragen.

\section{Hauptknoten und Hauptzonen.}

Als Hauptknoten treten im Bilde hervor: $\bmod , c z y n a$; in zweiter Linie $b \lambda$.

Die anderen Formen stehen an Wichtigkeit zurück und haben keinen wesentlichen Einfluss auf die Entwickelung der Formen. Die Rolle der

1) Zur Reproduction gelangte nur die gnomonische Projection au $c=0(001$ Taf. XIII, Fig. 21.

2) Die Reproduction giebt diese Retlexe schwarz wieder. 
einzelnen Formen in den wichtigsten Zonen ist aus der Discussion der Zahlenreihen ersichtlich. Aus ihr und den Projectionsbildern können wir den Hauptflächen eine relative Rangordnung zuschreiben, die etwa die obige ist.

Die Reflexe der krummen Flächen wurden alle in das gleiche Bild zusammengetragen. Aus diesen Bildern bestätigt sich, dass diese Lichtzüge in den Hauptzonen des Anglesits verlaufen, und dass sie sich in den Hauptknoten schneiden, und dass wir in ihnen ein Mittel haben, die Hauptknoten und -zonen zu finden.

Als Hauptknoten erscheinen danach die Flächenpunkte: mod, czyna.

Es sind im Wesentlichen dieselben, die sich aus den Gesammtbildern der beobachteten Einzelflächen ergeben haben. Beide Wege der Erkenntnis ergänzen und beslätigen sich gegenseitig. Auf die Einzelheiten in der Beurtheilung dieser Reflexzüge möge hier nicht näher eingegangen werden.

\section{Winkeltabellen.}

Jie nachstehenden Winkeltabellen wurden nach dem in Goldschmidt's Winkeltabellen gegebenen Schema gebildet und die Winkel, mit Ausnahme von denen für neue lormen, von dort entnommen. Es schien mir jedoch wünschenswerth, für den $\Lambda$ nglesit eine Winkeltabelle zu haben, die nicht nur für die Aufstellung 1. der Krystalle (Polflïche ist $c=0$ ), sondern auch für die Aufstellungen 2 (Polfläche $=b=\infty 0$ ) und 3 (Polfläche $=$ $a=0 \infty$ ) Geltung hat. Die Berechnung dieser Tabellen (für Aufstellung 2 und 3 lässt sich aus der Tabelle 1 leicht ableiten 1), und zwar:

bezeichnen wir die Winkel der $\Lambda$ ufstellung 1 mit $\varphi \varrho \xi \eta \xi_{0} \eta_{0}$

so gelten folgende Beziehungen:

\begin{tabular}{|c|c|c|}
\hline Aufstellung 1 & Aufstellung 2 & Aufstellung $\mathbf{3}$ \\
\hline $\begin{array}{l}\varphi=90^{0}-\xi_{0}^{\prime}=r_{0}^{\prime \prime} \\
\varrho=90^{0}-\eta^{\prime}=900-\xi^{\prime \prime} \\
\xi_{0}=90^{0}-r_{0^{\prime}}=900-\varphi^{\prime \prime} \\
r_{0}=\varphi^{\prime}=900-\xi_{0}^{\prime \prime} \\
\xi_{0}=90^{\prime \prime}-\varrho^{\prime}=r_{0}^{\prime \prime} \\
\eta=\xi^{\prime}=900-\rho^{\prime \prime}\end{array}$ & 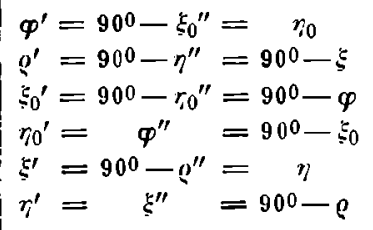 & $\begin{array}{l}\varphi^{\prime \prime}=90^{0}-\xi_{0}=\eta_{0}^{\prime} \\
\varrho^{\prime \prime}=90^{\prime}-\eta=90^{0}-\xi \\
\xi_{0}^{\prime \prime}=90^{0}-r_{0}=90^{0}-\varphi^{\prime} \\
\eta_{0}^{\prime \prime}=\varphi=90^{\circ}-\xi_{0}^{\prime} \\
\xi^{\prime \prime}=90^{\prime}-\varrho=\eta^{\prime} \\
\eta^{\prime \prime}=\xi=90^{\prime}-\varrho^{\prime}\end{array}$ \\
\hline
\end{tabular}

Wenn auch diese Umrechnungen einfach sind, so ist es doch für Jeden, der sich mit Messungen des Anglesits beschäftigt, bequemer, die Werthe für alle bekannten Formen ausgerechnet und in Tabellen bequem geordnet vor sich zu haben.

1) Gdt. Winkeltabellen 1897 , S. 8. 
Aufstellung 1: Polfläche $\mathrm{c}=0$.

Elemente.

\begin{tabular}{|c|c|c|c|c|c|}
\hline$a=0,7852$ & $\lg a=989498$ & $\lg a_{0}=978439$ & $\lg p_{0}=021541$ & $a_{0}=0,6089$ & $p_{0}=1,6421$ \\
\hline$c=1,2894$ & $\lg c=011039$ & $\lg b_{0}=988961$ & $\lg q_{0}=011039 b_{0}=0,7753$ & $q_{0}=1,2894$ \\
\hline
\end{tabular}

\begin{tabular}{|c|c|c|c|c|c|c|c|c|c|c|c|c|c|c|c|}
\hline Nr. & 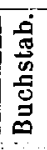 & 总 & ! & $\varphi$ & & 0 & $\xi_{0}$ & & 10 & $\xi$ & & $\eta$ & $\mid \begin{array}{c}x \\
\text { (Prismen) } \\
(x: y)\end{array}$ & $y$ & $\begin{array}{l}d= \\
\operatorname{tg} \varrho\end{array}$ \\
\hline 1 & $e$ & 0 & 001 & - & 10 & $\begin{array}{ll}0 & 0^{\prime}\end{array}$ & $\begin{array}{lll}0 & 0^{\prime}\end{array}$ & 00 & $\begin{array}{ll}0 & 0^{\prime}\end{array}$ & $\begin{array}{ll}00 & 0^{\prime}\end{array}$ & $0^{0}$ & $0 \quad 0^{\prime}$ & 0 & o & 0 \\
\hline 2 & $a$ & 000 & 010 & 00 & $0^{\prime}: 90$ & $0 \quad 0$ & $\downarrow$ & 90 & 0 & $D$ & 90 & 0 & D & $\infty$ & $\infty$ \\
\hline 3 & $b$ & $\infty 0$ & 100 & 90 & 0 & » & $90 \quad 0$ & 0 & 0 & $90 \quad 0$ & 0 & 0 & $\infty$ & 0 & 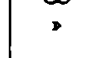 \\
\hline 4 & $M$ & 400 & 410 & 785 & & » & » & 90 & 0 & 7853 & 11 & 6. & 5,0943 & $\infty$ & " \\
\hline ; & $N$ & $3 \infty 0$ & 310 & 752 & & , & " & & 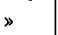 & 7520 & 14 & 40 & $\mathbf{3}, 8206$ & 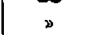 & " \\
\hline 6 & $O$ & 5 & 520 & 723 & & " & " & & $»$ & 7234 & 17 & 26 & 3,1839 & 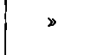 & * \\
\hline 7 & $\lambda$ & 200 & 210 & 68 & & » & » & & » & 6834 & 21 & 26 & 2,5471 & ע & " \\
\hline 8 & $P$ & $\frac{7}{4} \infty$ & 740 & $6: 5$ & & " & " & & » & i5 50 & 24 & 10 & 2,2987 & , & , \\
\hline 9 & $i$ & 3 & 320 & $62 \quad 2$ & & " & D & & 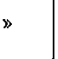 & $62 \quad 22$ & 27 & 38 & $1,9103$. & 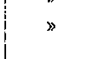 & " \\
\hline 10 & $O$ & 1900 & 10.7 .0 & 61 19 & & " & ळ & & 》 & $l_{61} 12$ & 28 & 48 & 1,8190 & 》 & " \\
\hline 11 & $Q$ & 300 & 430 & 5936 & & * & 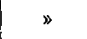 & & » & $930^{\circ}$ & 30 & 29 . & 1,6981 & " & » \\
\hline 12 & $\underline{x}$ & 50 & 540 & 575 & & " & $\gg$ & & 》 & $57 \quad 52$ & 32 & 8 & 1,5921 & 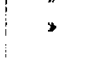 & . \\
\hline 13 & $R$ & ${ }_{3}^{10} \infty$ & 10.9 .0 & 5445 & & » & $\nu$ & & » & $34 \quad 45$ & 35 & 15 & 1,4151 & » & " \\
\hline 14 & $m$ & $\infty$ & 110 & 5151 & & " & * & & " & 5151. & 38 & 8. & 1,2736 & 》 & D \\
\hline 15 & $S$ & $\infty_{90}^{10}$ & 9.10 .0 & 485 & & $\Rightarrow$ & $D$ & $n$ & " & $48 \quad 54$ & 41 & 6 & 1,1462 & 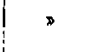 & 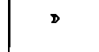 \\
\hline 16 & $T$ & $\infty \frac{8}{11}$ & 780 & 48 & & 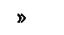 & $\triangleright$ & & » & 186 & 4.1 & 54 & 1,1143 & " & D \\
\hline 17 & $U$ & $\infty ?$ & 790 & 4443 & & $\gg$ & 》 & & » & 4443 & 45 & 16. & $0,9905$. & 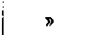 & D \\
\hline 18 & $h$ & $\infty$ & 340 & 4341 & & $»$ & " & s & $\Delta$ & $43 \quad 41$ & 46 & 19 & 0,9532 & " & » \\
\hline 19 & $\delta$ & $\infty ?$ & 230 & 4020 & & , & " & s & מ & 1020 & 49 & 40 & $0,8490^{\circ}$ & » & D \\
\hline 20 & $\gamma$ & $\infty \infty_{5}^{\frac{R}{8}}$ & 580 & $38 \quad 31$ & & 》 & $\$$ & s & \$ & $38 \quad 31$ & 51 & 29 & 0,7960 & $D$ & , \\
\hline 21 & $n$ & $\infty 2$ & 120 & $32 \quad 29$ & & $»$ & $"$ & " & " & $3229^{\circ}$ & 57 & 30. & 0,6368 & $"$ & , \\
\hline 22 & $x$ & $\infty 3$ & 4 & 0 & & 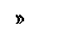 & $\gg$ & » & क & 23 & 67 & $\mathbf{0}$ & 45 & $D$ & $D$ \\
\hline $2: 3$ & $W$ & $\infty$ & 270 & 200 & & » & " & & » & 200 & 70 & 0 & 0,3639 & $\gg$ & 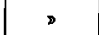 \\
\hline 24 & $A$ & $0_{\mathrm{T}^{1}}^{1-}$ & 0.1 .16 & 00 & & $36^{\circ}$ & $\begin{array}{ll}0 & 0\end{array}$ & & 36. & $0 \quad 0$ & 4 & 36 . & 0 & 0,0806 & 0,0806 \\
\hline 25 & « & $0 \frac{1}{8}$ & 018 & $\infty$ & $\mathbf{9}$ & 0 & » & 9 & 9 . & 》 & 9 & 9 . & $\rrbracket$ & $0,1611$. & $1,1611$. \\
\hline 26 & $j$ & $0 \frac{2}{11}$ & 0.2 .11 & \$ & 13 & 11. & " & 13 & 11 . & 》 & 13 & 11. & $\Rightarrow$ & 0,2344 . & $0,2344$. \\
\hline 27 & $B$ & $0 \%$ & 029 & D & 13 & $59^{\circ}$ & " & 15 & $\mathbf{5 9}$. & » & 13 & 59. & 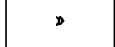 & 0,2865 . & 0,2865 \\
\hline 28 & $v$ & 0.1 & 013 & 》 & 23 & 15 & $\Rightarrow$ & 23 & 13 & $\gg$ & 23 & $15^{\circ}$ & D & 8 & 0,4298 \\
\hline 29 & $\varphi$ & $0 \frac{3}{2}$ & 012 & D & 32 & 48 & D & 32 & $48^{\circ}$ & » & 32 & 48. & " & 47 & 0,6447 \\
\hline 30 & $x$ & $0 . \frac{3}{5}$ & 035 & 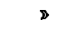 & 37 & 43. & \$ & 37 & 43. & $»$ & 37 & $43^{\circ}$ & s & 0,7736 & 0,7736 \\
\hline 31 & 0 & 01 & 011 & 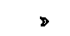 & 52 & 12 & $D$ & 52 & 12 & " & i52 & 12 & $\triangleright$ & 2894 & 1,2894 \\
\hline 32 & $\vartheta$ & 02 & 021 & $\gg$ & 68 & 48 & D & 68 & 48 & , & 68 & 48 & s & $\begin{array}{r}1,2084 \\
9 ; 388\end{array}$ & 2,5788 \\
\hline $3 \mathbf{3}$ & $\beta$ & 03 & 031 & s & 75 & 30 & \& & 75 & $30^{\circ}$ & 》 & j 73 & $30^{\circ}$ & 》 & 3.8682 & 3,8682 \\
\hline
\end{tabular}




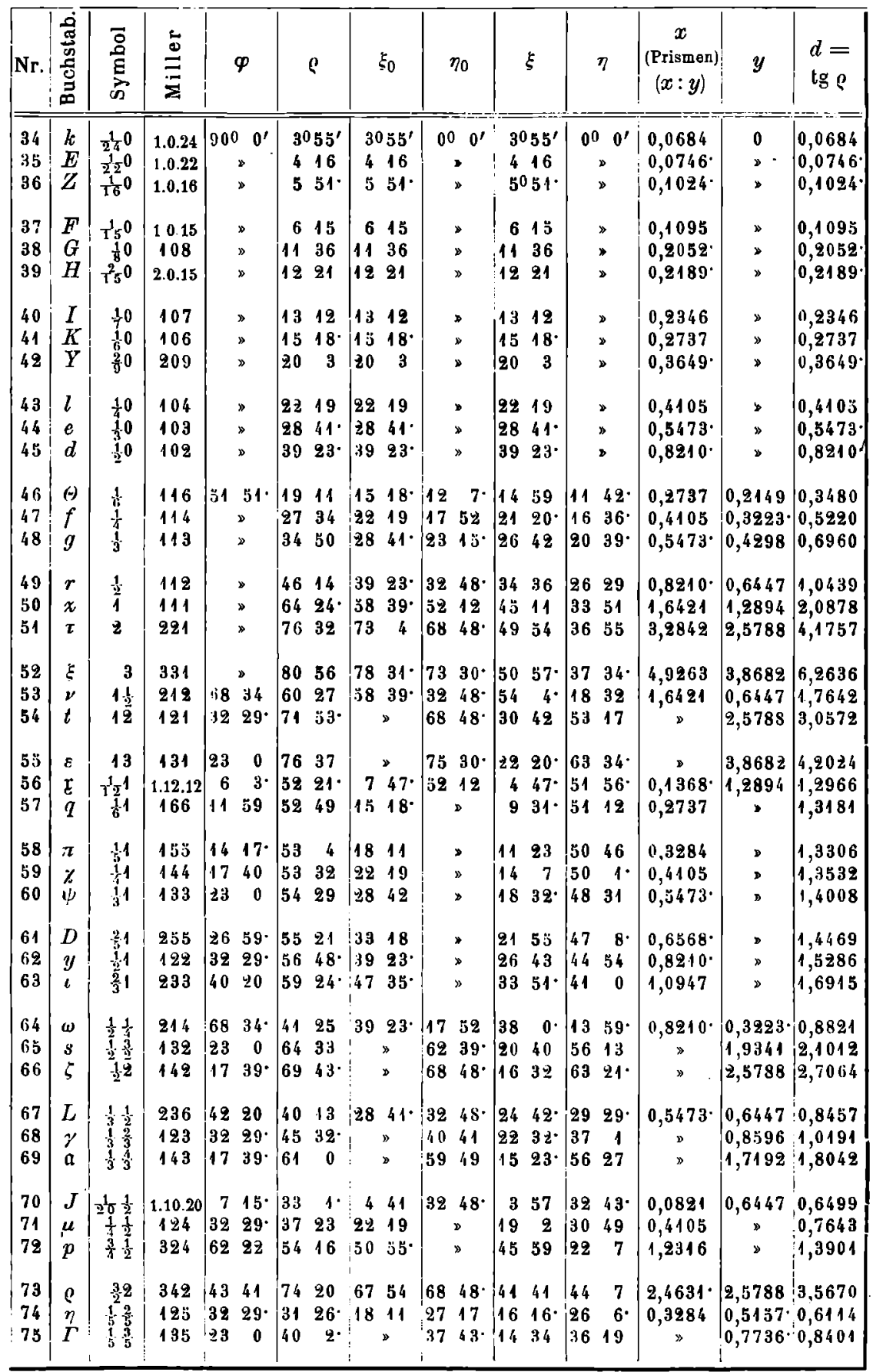




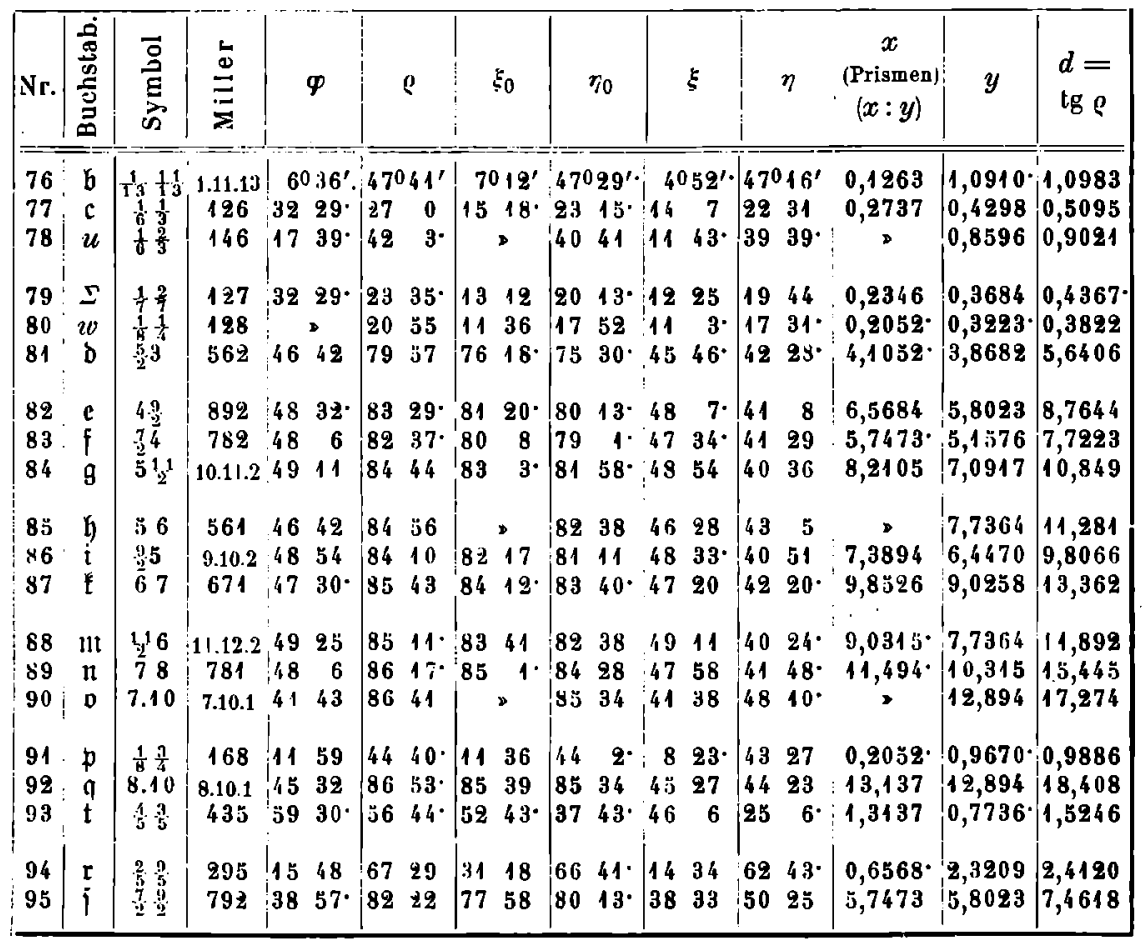

Aufstellung 2: Polfläch $\mathrm{b}=\infty 0$.

Elemente.

\begin{tabular}{|l|l|l|l|l|l|}
\hline$a^{\prime}=1,2894$ & $\lg a^{\prime}=011039$ & $\lg a_{0}{ }^{\prime}=010502$ & $\lg p_{0}{ }^{\prime}=989498$ & $a_{0}{ }^{\prime}=1,2736$ & $p_{0}{ }^{\prime}=0,7652$ \\
\hdashline$c^{\prime}=0,6090$ & $\lg c^{\prime}=978459$ & $\lg b_{0}{ }^{\prime}=021541$ & $\lg q_{0}^{\prime}=978459$ & $b_{0}{ }^{\prime}=1,6421$ & $q_{0}{ }^{\prime}=0,6090$ \\
\hline
\end{tabular}

\begin{tabular}{|c|c|c|c|c|c|c|c|c|c|c|c|c|c|c|c|c|c|}
\hline Nr. & 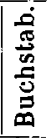 & 总 & $\stackrel{\grave{\Xi}}{\Xi}$ & & $p^{\prime}$ & $\varrho^{\prime}$ & & $\xi_{0}^{\prime}$ & & $\eta_{0^{\prime}}$ & & $\xi$ & $\eta$ & & $\mid \begin{array}{c}x^{\prime} \\
(\text { Prismen) } \\
\left(x^{\prime}: y^{\prime}\right)\end{array}$ & $y^{\prime}$ & $\begin{array}{l}d^{\prime}= \\
\operatorname{tg} \varrho\end{array}$ \\
\hline 1 & $c$ & $0 \infty 0$ & 010 & 00 & $0^{\prime}$ & 900 & $0^{\prime}$ & 900 & $0^{\prime} !$ & $900 \mathrm{O}^{\prime}$ & 00 & $0^{\prime}$ & 900 & $0^{\prime}$ & 0 & $\infty$ & $\infty$ \\
\hline 2 & $a$ & $\infty 0$ & 100 & 90 & 0 & $D$ & & » & & , & 90 & 0 & 0 & 0 & $\infty$ & 0 & $"$ \\
\hline 3 & $b$ & 0 & 001 & o & 0 & 0 & 0 & & 0 & $\begin{array}{ll}0 & 0\end{array}$ & 0 & 0 & D & & 0 & " & 0 \\
\hline 4 & $M$ & 40 & 10 & 90 & 0 & 11 & $6^{\circ}$ & 11 & 6 & » & 11 & $6^{\circ}$ & D & & & » & 0,1 \\
\hline 5 & $N$ & $\frac{10}{3} 0$ & 103 & & $\triangleright$ & 4 & 40 & 144 & & 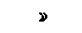 & 14 & 40 & D & & & 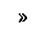 & \\
\hline 6 & 0 & 20 & 205 & & D & 17 & 26 & 172 & & s & 17 & 26 & s & & & D & 0, \\
\hline 7 & $\lambda$ & 10 & 102 & & » & 2 & 26 & 212 & & , & 21 & 2 & , & & & » & 0.99 \\
\hline 8 & $P$ & $\frac{4}{7} 0$ & 4 & & 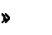 & 241 & 10 & 241 & & » & 24 & 11 & 8 & & & 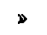 & 7 \\
\hline 9 & $i$ & $\frac{2}{3} 0$ & 203 & & $\nabla$ & $27:$ & 38 & 273 & & 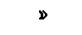 & 27 & 38 & 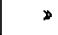 & &, $5234^{\circ}$ & 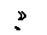 & 0,5234 \\
\hline 10 & $C$ & 70 & & & & 4 & 48 & 284 & 8 & $D$ & 28 & 4 & ע & & & 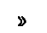 & \\
\hline 11 & $Q$ & $\begin{array}{l}0 \\
3 \\
3\end{array}$ & & & 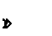 & 2 & $29^{\circ}$ & 302 & & 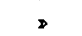 & 30 & 29 & 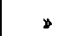 & & & D & \\
\hline 12 & $\mathcal{\Omega}$ & 30 & 405 & & > & 32 & 8 & 32 & 8 & 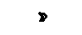 & 32 & 8 & $\triangleright$ & & $0,6281$. & $\gg$ & 0,6281 \\
\hline
\end{tabular}




\begin{tabular}{|c|c|c|c|c|c|c|c|c|c|c|c|c|c|c|c|c|c|c|}
\hline $\mathrm{Nr}$. & 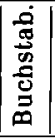 & 煦 & $\stackrel{\bar{\Xi}}{\equiv}$ & & $p^{\prime}$ & & $\varrho^{\prime}$ & & $\xi_{0}^{\prime}$ & $\eta_{0}$ & $0^{\prime}$ & & $\xi$ & $\eta^{\prime}$ & & $\begin{array}{c}x^{\prime} \\
\text { (Prismen) } \\
\left(x^{\prime}: y^{\prime}\right)\end{array}$ & $y^{\prime}$ & $\begin{array}{l}d^{\prime}= \\
\operatorname{tg} \varrho\end{array}$ \\
\hline 13 & $R$ & $\frac{9}{10} 0$ & 9.0 .10 & 900 & $0^{\prime}$ & 350 & $15^{\prime}$ & & $015^{\prime}$ & & $0^{\prime}$ & & $15^{\prime}$ & 00 & & 0,7067 & 0 & 0,7067 \\
\hline 14 & $m$ & 10 & 101 & & 2 & 38 & 8 . & & 8 & , & . & & 8. & $»$ & & 0,7852 & , & $0,7 \times 52$ \\
\hline 15 & $S$ & 100 & io.0.9 & & 2 & 41 & 6 & 41 & 6 & D & & & & 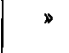 & & $0,8724^{\circ}$ & . & 0,8724 \\
\hline 16 & $T$ & 80 & 807 & & 8 & 41 & 54 & 41 & 54 & D & 。 & 415 & 54 & $D$ & & 0,8973 & » & 0,8973 \\
\hline 17 & $U$ & 30 & 907 & & » & 45 & $16^{\circ}$ & 43 & $16^{\circ}$ & D & o & 451 & $16^{\circ}$ & D & & $94^{\circ}$ & » & 1,0094 \\
\hline 18 & $h$ & $\frac{1}{3} 0$ & 403 & & - & 46 & 19 & 46 & 19 & 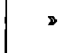 & 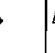 & 461 & 19 & D & & 1,0469 & $\star$ & 1,0469 \\
\hline 19 & $\delta$ & $\frac{3}{2} 0$ & 302 & & " & 49 & 40 & 49 & 40 & ఎ & 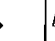 & 494 & 40 & $\gg$ & & 1,1778 & * & 1,1778 \\
\hline 20 & $\boldsymbol{V}$ & 80 & 805 & & $»$ & 51 & 29 & 51 & 29 & » & 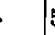 & 51 & 29 & 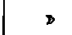 & & 3 & . & 1,2563 \\
\hline 21 & $n$ & 20 & 201 & & ע & 57 & $30^{\circ}$ & 57 & 30 & . & & 573 & $30^{\circ}$ & 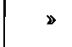 & & 1,5704 & $"$ & 1,5704 \\
\hline 22 & $x$ & 30 & 301 & & & 67 & 0 & 67 & 0 & $"$ & & 67 & 0 & s & & 3556 & 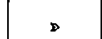 & 2,3556 \\
\hline 23 & $W$ & $\frac{7}{2} 0$ & 702 & & 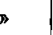 & 70 & 0 & 70 & 0 & » & & 70 & 0 & 2 & & & 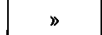 & 2,7482 \\
\hline 24 & $A$ & $\infty .16$ & 1.16 .0 & 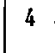 & 36 . & 90 & 0 & 90 & 0 & & 0 & 43 & 36. & 852 & & 0,0806 & $\infty$ & $\infty$ \\
\hline 25 & $r$ & $\infty 8$ & 180 & 9 & 9. & & $\triangleright$ & & $\triangle$ & " & & 9 & 9. & 8050 & & 0,1612 & 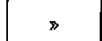 & » \\
\hline 26 & $j$ & $\infty \frac{11}{2}$ & 2.11 .0 & 13 & 11. & & 》 & & $"$ & D & & 131 & 11. & 76 4! & $8 \cdot$ & & D & " \\
\hline 27 & $B$ & $\infty_{2}^{9}$ & 290 & 15 & 59 & & $\gg$ & & b & 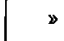 & & 155 & $59 \cdot$ & 74 & 0. & 0,2890 & $D$ & $\gg$ \\
\hline 28 & $v$ & $\infty 3$ & 130 & 23 & 15. & & > & & . & * & & 231 & 15. & 66 & & 6 & ע & . \\
\hline 29 & $\boldsymbol{\varphi}$ & $\infty 2$ & 120 & 32 & 48. & & ע & & $D$ & $D$ & & 324 & 48. & $37 \quad 11$ & & 7 & $\gg$ & $\triangleright$ \\
\hline 30 & $x$ & $\infty$ & 350 & 37 & 43. & & 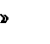 & & $\triangleright$ & 》 & & 374 & $43 \cdot$ & $\$ 216$ & & $0,7736^{\circ}$ & $D$ & . \\
\hline 31 & $o$ & $\infty$ & 110 & 52 & 12 & & 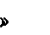 & & » & 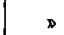 & & 521 & 12 & $37 \quad 48$ & & 1,2894 & » & $\nu$ \\
\hline 32 & $\vartheta$ & 200 & 210 & 68 & $48^{\circ}$ & & $\nu$ & & 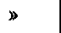 & 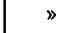 & & 684 & 48. & $21 \quad 11$ & & 8 & " & $"$ \\
\hline 33 & $\beta$ & $3 \infty$ & 310 & 75 & $30^{\circ}$ & & × & & » & D & & 753 & $30 \cdot$ & 1429 & & 3,8682 & " & $»$ \\
\hline 34 & $k$ & 0.24 & .1 & 0 & 0 & 86 & $\mathbf{5}$ & 0 & 0 & & 5 & 0 & 0 & 86 & 5 & 0 & 14,616 & 14,516 \\
\hline $3 \ddot{3}$ & $E$ & 0.22 & 0 & & » & $8: i$ & 44 & & 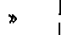 & & 44 & 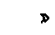 & $\rightarrow$ & 8544 & & \# & 13,398 & 13,398 \\
\hline 36 & $Z$ & 0.16 & 0.16 .1 & & " & 84 & 8. & & $D$ & 84 & 8. & » & 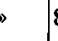 & 848 & 8. & $»$ & 9,7440 & 9,7440 \\
\hline 37 & $F$ & $0.1 \ddot{3}$ & 0. & & 》 & 83 & 45 & & D & 834 & 45 & , & 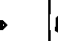 & 8345 & & 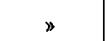 & 50 & 50 \\
\hline 38 & $G$ & 08 & 08 & & " & 78 & 24 & & 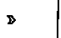 & 782 & 24 & » & 》 & $78 \quad 24$ & & $"$ & 0 & 4,8720 \\
\hline 39 & $H$ & $012^{15}$ & 0.15 .2 & & $\gg$ & 77 & 39 & & \% & 773 & 39 & • & 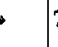 & 7739 & & 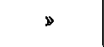 & 4,5675 & 4,5675 \\
\hline 40 & $J$ & 07 & 07 & & » & 76 & 48 & & , & 4 & 48 & » & 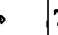 & $\begin{array}{ll}76 \quad 48 \\
\end{array}$ & & 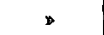 & & \\
\hline 41 & $K$ & 0 & 10 & & D & 74 & 41 & & D & $74 \quad 4$ & $44^{\circ}$ & » & 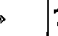 & $74 \quad 41$ & & 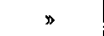 & & 40 \\
\hline 42 & $Y$ & $0 \frac{9}{2}$ & 092 & & * & 69 & 57 & & $\gg$ & 695 & 57 & » & 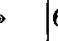 & 6957 & & $D$ & 9,7405 & 2,7405 \\
\hline 43 & $l$ & 04 & 011 & & » & 67 & 41 & & , & 674 & 41 & $x$ & . & $67 \quad 41$ & & . & 60 & 360 \\
\hline 4 & $e$ & 0 & & & 》 & 61 & 18 & & $D$ & 610 & $18^{\circ}$ & . & . & $61 \quad 18$ & & $D$ & & 270 \\
\hline 45 & $d$ & 02 & 021 & & 》 & 50 & $36^{\circ}$ & & 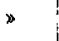 & 503 & $36^{\circ}$ & 》 & 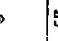 & 503 & & $D$ & 1,2180 & 1,2180 \\
\hline 40 & $\theta$ & 16 & 1 & 12 & $7 \cdot$ & $7:$ & 1 & 38 & 8. & 744 & $1 \cdot 1$ & 114 & $42 \cdot$ & $70 \quad 45$ & & 0,7852 & & 65 \\
\hline 4 & $f$ & 1 & 14 & 17 & 52 & 68 & 40 & & " & 674 & $1 \cdot 1$ & $16:$ & $36 \cdot$ & $622 f$ & & $\pi$ & & \\
\hline 48 & $g$ & 13 & 131 & 23 & 15 & 63 & 18 & & 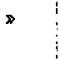 & 61 & $18 \cdot 2$ & 203 & 39. & 3510 & & 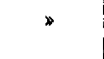 & 1,8270 & 1,9883 \\
\hline $4 !$ & $r$ & 12 & I & 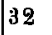 & $48^{\circ}$ & 55 & 24 & & 》 & 303 & $36 \cdot 2$ & 268 & 29 & 634 & & , & 80 & \\
\hline 50 & $x$ & 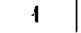 & 1 & 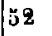 & 12 & 44 & 49 & & D & $31 \quad 2$ & 20.3 & 335 & 51 & 2535 & & 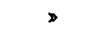 & & \\
\hline 31 & $\boldsymbol{\tau}$ & $1 \frac{1}{2}$ & 212 & 68 & 48. & 40 & 6 & & $\gg$ & 165 & 56 & 365 & 55 & 1328 & & $D$ & 0,3045 & 0,8421 \\
\hline 52 & $\xi$ & $1 \frac{1}{3}$ & 31 & 75 & 30. & 39 & 2. & & 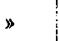 & 112 & $28 \cdot$ & $37:$ & 34. & 9 & 4 & & & 0,8109 \\
\hline : & $v$ & $2_{2}^{1}$ & \begin{tabular}{l|l}
122 \\
\end{tabular} & 32 & $45^{\circ}$ & $3 \ddot{3}$ & $55^{\circ}$ & 21 & 26 & $\begin{array}{ll}31 & 2\end{array}$ & $20 \cdot 1$ & 183 & 32 & 293 & & & 0,6090 & 0,7245 \\
\hline 5 & $t$ & 21 & ! 211 & 68 & 18 & 59 & 18 & 57 & 30. & 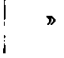 & & 531 & 17 & 18 & $6^{\circ}$ & 1,5704 & 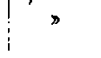 & 1,6191 \\
\hline
\end{tabular}




\begin{tabular}{|c|c|c|c|c|c|c|c|c|c|c|c|c|c|c|c|c|c|}
\hline Nr. & 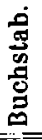 & 昌 & $\begin{array}{l}\stackrel{4}{\Xi} \\
\Xi \\
\Xi\end{array}$ & & $\boldsymbol{\varphi}^{\prime}$ & & $e^{\prime}$ & & $\xi_{0}^{\prime}$ & $\eta_{0}^{\prime}$ & & $\xi^{\prime}$ & & $\eta^{\prime}$ & $\left\{\begin{array}{c}x^{\prime} \\
(\text { Prismen }) \\
\left(x^{\prime}: y^{\prime}\right)\end{array}\right.$ & $y^{\prime}$ & $\begin{array}{l}d^{\prime}= \\
\operatorname{tg} \rho^{\prime}\end{array}$ \\
\hline 55 & $\varepsilon$ & 31 & 311 & $75^{0}$ & $030^{\prime}$ & 67 & $7039^{\prime}$ & 670 & $000^{\prime}$ & $31020^{\prime}$ & $63^{0}$ & $034^{\prime}$ & 130 & $023^{\prime}$ & 2,3556 & 0,6090 &, 4741 \\
\hline 56 & $\tau$ & 12. & 12.12.1 & & 12 & 55 & 12 & 83 & $56^{\circ}$ & $82 \quad 12$ & 31 & $56^{\circ}$ & 37 & 38. & 24 & & 11,932 \\
\hline 57 & $q$ & 6 & 661 & & $>$ & 80 & $28^{\circ}$ & 78 & 01 & $7441^{\circ}$ & 51 & 12 & 37 & 11 & $4,7+12$ & 540 & $5,9600^{\circ}$ \\
\hline 58 & $\pi$ & $\mathbf{5}$ & $j^{j}$ & & , & 78 & 37 & 75 & 42. & $71 \quad 49$ & 50 & $46^{\circ}$ & 36 & $55^{\circ}$ & 60 & 50 & 4,004 \\
\hline 59 & $x$ & 4 & 41 & & , & 75 & 52. & 72 & 20 & $67 \quad 41$ & 50 & 01. & 36 & 28 & 108 & 360 &, 9740 \\
\hline 60 & $\psi$ & 3 & 331 & & * & 71 & $27^{\circ}$ & 67 & 0 & 6118 & 48 & 31 & 35 & 31 & 2,3556 & 1,8270 & 2,9815 \\
\hline 61 & $D$ & $\frac{5}{2}$ & 552 & & , & 68 & 5 & 63 & 0. & $56 \quad 42$ & 47 & 08. & 34 & 39 & 30 & 225 & $2, \pi 0066$ \\
\hline $6 z$ & $y$ & $\mathbf{q}$ & 221 & & > & 63 & 17 & 57 & 30. & $5036^{\circ}$ & 44 & 54 & 33 & 11 & 04 & 1,2180 & 1,9869 \\
\hline 63 & $\iota$ & $\frac{3}{2}$ & 332 & & - & 56 & 8 & 49 & 10 & $4224^{\circ}$ & 41 & $00^{\circ}$ & 30 & 35. & 1,1778 & 0,9135 & 1,4994 \\
\hline 64 & $\omega$ & $\frac{1}{2} 2$ & $z$ & 17 & 52 & 51 & 59. & 21 & 26 & $5036^{\circ}$ & 13 & $59^{\circ}$ & 48 & 35 & & ,2180 &, 2795 \\
\hline 65 & $s$ & 32 & 34 & 62 & 39. & 69 & 20 & 67 & 0 & $\checkmark$ & 56 & 13 & 25 & 27 & & $>$ &, 6511 \\
\hline 66 & $\zeta$ & 42 & 421 & 68 & 48 & 73 & 28 & 72 & $20^{\circ}$ & $>$ & 63 & 21 & 20 & $16^{\circ}$ & & - & 3,1652 \\
\hline 67 & $L$ & $\frac{3}{2} 3$ & 2 & 32 & $48^{\circ}$ & 65 & 17 & 49 & 40 & $6118^{\circ}$ & 29 & $29^{\circ}$ & 49 & $46^{\circ}$ & &, 8270 & 2,1725 \\
\hline 68 & $\gamma$ & 93 & 31 & 40 & 41 & 67 & 27 . & 37 & $30^{\circ}$ & , & 37 & 01 & 44 & 27. & & , &, 4093 \\
\hline 69 & $a$ & 43 & 431 & 59 & 49 & 74 & $36^{\circ}$ & 78 & $20^{\circ}$ & $\triangleright$ & 56 & 26 & 29 & 0 & 3,1408 & - &, 6326 \\
\hline 70 & $\mathfrak{F}$ & 10.20 & 10.20 .1 & 32 & 48. & 86 & 9 & 82 & $44^{\circ}$ & $8518^{\circ}$ & 32 & $43^{\circ}$ & 56 & 58. & & 30 & 488 \\
\hline 71 & $\mu$ & 24 & 241 & & $>$ & 70 & 58 & 57 & 30. & $67 \quad 41$ & 30 & 49 & 52 & 36. & & 960 & $387^{\circ}$ \\
\hline 72 & $p$ & 5 & 243 & & $>$ & 44 & 0. & 27 & 38 & $39 \quad 4^{\circ}$ & 22 & 07 & 35 & 44 & $34^{\circ}$ & 0,8120 & 0,$96 ; 9$ \\
\hline 73 & 0 & $4 \frac{2}{3}$ & 4 & 68 & 48. & 48 & 19 & 36 & 19 & 22 & 44 & 7 . & 15 & 40 & & & \\
\hline 74 & $\eta$ & 25 & 251 & 27 & 17 & 73 & $43-$ & 57 & $30^{\circ}$ & $71 \quad 49$ & 26 & 6 , & 54 & $3 y \cdot$ & & 0450 & 3,4234 \\
\hline 75 & $\Gamma$ & 35 & 351 & 37 & $43^{\circ}$ & 75 & 26 & 67 & 0 & > & 36 & $19^{\circ}$ & 49 & 87 & 2,3556 & , &, 8483 \\
\hline 76 & $\mathfrak{b}$ & 41.13 & 11.13.1 & 47 & $29^{\circ}$ & 85 & 7 & 83 & 23. & $8 z \quad 48$ & 47 & 16 & 42 & 19 & & 70 & 727 \\
\hline 77 & c & 2 & 26 & 23 & 15 & 75 & 53 & 57 & $30^{\circ}$ & $74 \quad 41$ & 2 & 31 & 63 & 0 & 4 & 6540 & 861 \\
\hline 78 & $u$ & 46 & 461 & 40 & 41 & 78 & $16^{\circ}$ & 72 & $20^{\circ}$ & $>$ & 39 & $39^{\circ}$ & 47 & $56^{\circ}$ & 08 & $D$ & 4,8184 \\
\hline 79 & $y$ & 27 & 71 & 20 & 13. & 77 & 35 & 57 & 30. & $76 \quad 48$ & 19 & 44 & 66 & $24^{\circ}$ & 104 & 30 & 21. \\
\hline 80 & $w$ & 28 & $T^{2}-5$ & 17 & 58 & 78 & 56 & & 》 & $\begin{array}{ll}78 & 24\end{array}$ & 17 & 31. & 69 & 5. & > & 20 & $780^{\circ}$ \\
\hline 81 & $\delta$ & (i) 2 & 625 & 75 & $30^{\circ}$ & 44 & 13 & 43 & 18 & 1341 & 42 & 28. & 10 & 3 & 9422 & 2436 & 0,9733 \\
\hline 8 & $e$ & & & 80 & $13^{*}$ & 41 & 52 . & 41 & 27 & $839^{\circ}$ & 41 & 8 & 6 & $30^{\circ}$ & & & \\
\hline$\times 3$ & f & & 827 & 79 & 1 . & 42 & 25 & 41 & 54 & 952 & 41 & 29 & 7 & 22. & $3 \cdot$ & 40 & 39 \\
\hline 84 & $\mathfrak{g}$ & $11 \frac{1}{5}$ & 11.2 .10 & & $58^{\circ}$ & 41 & 6 & 40 & 49 & $656^{\circ}$ & 40 & $36^{\circ}$ & $\mathbf{5}$ & 16 & 0,8637 & 0,1218 & $0,87 \geq 3$. \\
\hline 85 & $\mathfrak{h}$ & & 615 & 82 & 38 & 43 & 32 & 43 & 18 & & 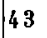 & $\check{3}$ & 5 & 4 & & & \\
\hline 86 & i & 102 & 10.2 .9 & 81 & 11 & 41 & $26^{\circ}$ & 41 & 6 & 742. & 40 & 51 & 5 & $49^{\circ}$ & $24^{\circ}$ & 0,1353 & $0,88: 9$ \\
\hline 87 & $\mathfrak{k}$ & 7 & 716 & 83 & $40^{\circ}$ & 42 & 40 & 48 & $29^{\circ}$ & 5 47 & 42 & $20^{\circ}$ & 4 & 17 & 0,91 i $0^{\circ}$ & 0,1015 & 0,9217 \\
\hline 88 & $\mathrm{wt}$ & $\frac{1}{1}+3^{2}$ & 10.2 .11 & 82 & 38 & 40 & 49 & 40 & 35 & $6 \quad 19$ & 40 & $24^{\circ}$ & 4 & $48^{\circ}$ & & & \\
\hline 85 & $\pi$ & & 81 & 84 & 28 & 42 & 2 & 41 & 54 & $458^{\circ}$ & 41 & 48. & 3 & $4 z^{\circ}$ & $73 \cdot$ & 0,0870 & $014^{\circ}$ \\
\hline 90 & $\mathfrak{v}$ & $6 \frac{1}{7}$ & 10.1 .7 & 85 & 34 & 48 & 22 & 47 & 17 & > & 48 & $10^{\circ}$ & $\mathbf{3}$ & 19 & 1,1217 & $\triangleright$ & 1,0862 \\
\hline 91 & $\mathfrak{p}$ & 6 & 6 & 44 & & 81 & 36 & 78 & 1 & $78 \quad 24$ & 43 & 27 & 43 & 19. & & 20 & \\
\hline 92 & $q$ & $\frac{5}{4}$ & 10.1 .5 & 85 & 34 & 44 & 33 & 44 & 28 & 421 & 44 & 23 & 3 & $6^{\circ}$ & &, 0761 & 0,9844 \\
\hline 93 & $\mathrm{t}$ & $\frac{3}{4}$ & 354 & 37 & 43 & 43 & 51 & 30 & 29 & $37 \quad 16$ & 25 & 6. & 33 & 15 & 0,5889 & 0,7612 & $0,9567^{\circ}$ \\
\hline 94 & $\mathfrak{r}$ & $9 \frac{5}{2}$ & 95 & 66 & $41^{\circ}$ & 75 & 26 & 74 & 12. & $\begin{array}{ll}56 & 42\end{array}$ & 62 & $43^{\circ}$ & 22 & 31 & & & 2 \\
\hline 93 & 5 & & $9 z 7$ & 80 & $13^{\circ}$ & 51 & 27 & 51 & $2 \cdot$ & 12 & 50 & 25 & 7 & $\mathbf{3 8}$ & 94. & 0,1740 & 1 \\
\hline
\end{tabular}


Aufetellung 3: Polfläche $a=000$.

Elemente.

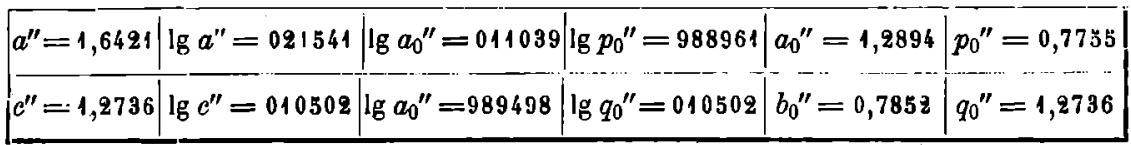

\begin{tabular}{|c|c|c|c|c|c|c|c|c|c|c|c|c|c|c|c|c|c|}
\hline $\mathrm{Nr}$. & 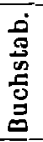 & $\frac{\frac{0}{8}}{\frac{0}{E}}$ & $\stackrel{\breve{d}}{\equiv}$ & & $g^{\prime \prime}$ & & ? & & $0^{\prime \prime}$ & & 60 & & $\xi^{\prime \prime}$ & $\eta^{\prime \prime}$ & $\begin{array}{c}x^{\prime \prime} \\
\text { (Prismen) } \\
\left(x^{\prime \prime}: y^{\prime \prime}\right)\end{array}$ & $y^{\prime \prime}$ & $\begin{array}{l}d^{\prime \prime}= \\
\operatorname{tg} \varrho^{\prime \prime}\end{array}$ \\
\hline 1 & $c$ & $\infty 0$ & 100 & 1900 & $0^{\prime}$ & 900 & $0^{\prime}$ & 1900 & $0^{\prime}$ & & — & 90 & $\begin{array}{ll}0 & 0^{\prime}\end{array}$ & $0^{0} 0^{\prime}$ & $\infty$ & 0 & $\infty$ \\
\hline 2 & $a$ & 0 & 001 & & - & o & 0 & 0 & 0 & 00 & $00^{\prime}$ & 0 & o & $D$ & 0 & , & 0 \\
\hline $\mathbf{3}$ & $b$ & $0 \infty$ & 010 & 0 & 0 & 90 & 0 & 90 & 0 & 90 & 0 & & D & $90 \quad 0$ & 3 & $\infty$ & $\infty$ \\
\hline 4 & $M$ & 04 & 041 & & > & 78 & $53 \cdot$ & 0 & 0 & 78 & 53. & & , & 7853. & , & 5,0944 & 5,0944 \\
\hline $\mathbf{5}$ & $N$ & 03 & 031 & & 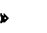 & 75 & 20 & & $\gg$ & 75 & 20 & & 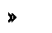 & 7520 & $\nabla$ & 3,8208 & 3,8208 \\
\hline 6 & 0 & 0.5 & 052 & & 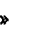 & 72 & 34 & & D & 72 & 34 & & 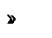 & 7234 & $D$ & 3,1840 & 3,1840 \\
\hline 7 & $\lambda$ & 02 & 021 & & , & 68 & 34 & & » & 68 & 34 & & D & $\begin{array}{ll}68 & 34\end{array}$ & $\triangleright$ & 2,3479 & 2,3472 \\
\hline 8 & $P$ & $0 \frac{7}{4}$ & 074 & & 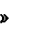 & 65 & 50 & & 》 & 65 & $\mathbf{5 0}$ & & $D$ & 6550 & 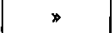 & 2,2288 & 2,2288 \\
\hline 9 & $i$ & $0 \frac{3}{2}$ & 032 & & " & 62 & 22 & & 》 & 62 & $\mathbf{2 z}$ & & , & 6222 & $\triangleright$ & 1,9104 & $1,91 \cap 4$ \\
\hline 10 & $C$ & 010 & 0.10 .7 & & 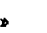 & 61 & 12 & & 》 & 61 & 12 & & 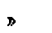 & 6112 & 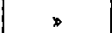 & 1,8194 & 1,8194 \\
\hline 11 & $Q$ & $0 \frac{1}{3}$ & $0 \div 3$ & & $»$ & 59 & 30 & & 》 & 59 & 30 & & * & 5930 & 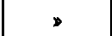 & 1,6981 & 1,6981 \\
\hline 12 & $S$ & $0 \frac{5}{4}$ & 054 & & > & 57 & 52 & & $\triangleright$ & 57 & 52 & & 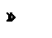 & 5752 & , & 1,5920 & 1,5920 \\
\hline 13 & $R$ & 010 & 0.10 .9 & & > & 54 & 45 & & 》 & 54 & 45 & & > & 3445 & D & 1,4151 & 1,4151 \\
\hline 14 & $m$ & 01 & 011 & $i$ & ૪ & 51 & 51. & & \$ & 51 & $51^{\circ}$ & & $D$ & $5151^{\circ}$ & D & 1,2736 & 1,2736 \\
\hline 15 & $S$ & $0 \frac{9}{10}$ & 0.9 .10 & & " & 48 & 54 & & $\triangleright$ & 48 & 54 & & $\triangleright$ & 4834 & $\triangleright$ & 1,1462 & 1,1462 \\
\hline 16 & $T$ & $0 \frac{7}{8}$ & 078 & & » & 48 & 6 & & » & 48 & 6 & & 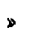 & 48 & $\triangle$ & 1,1144 & 1,1144 \\
\hline 17 & $U$ & 07 & 079 & & » & 44 & 43. & & » & 44 & 43. & & $\Delta$ & $4443^{\circ}$ & $\triangleright$ & 0,9906 & 0,9906 \\
\hline 18 & $h$ & $0: \frac{1}{4}$ & 034 & & » & 34 & $\{1$ & & 》 & 43 & 41 & & $\Rightarrow$ & 4341 & 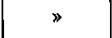 & 0,9553 & 0,9552 \\
\hline 19 & $\delta$ & $0 \frac{2}{3}$ & 023 & & » & 40 & 20 & & » & 40 & 20 & & D & 4020 & 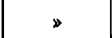 & $0,8 / 90$. & $0,8490^{\circ}$ \\
\hline 20 & $V$ & $0 \frac{5}{6}$ & 058 & & " & 38 & $: 1$ & & 》 & 38 & 31 & & $»$ & $38 \quad 31$ & $"$ & 0,7960 & 0,7960 \\
\hline 21 & $n$ & $0 \frac{1}{2}$ & 012 & & $\$$ & 23 & $2 .^{\circ}$ & & $\gg$ & 32 & $29^{\circ}$ & & $\gg$ & $322^{\circ}$ & $D$ & 0,6368 & 0,6368 \\
\hline 22 & $x$ & 0.1 & 013 & & " & 23 & 0 & & » & 23 & 0 & & " & 23 & * & 0,4245 & 0,4545 \\
\hline 23 & $W$ & $0 \frac{2}{7}$ & 027 & & ע & 20 & 0 & & » & $\Xi_{0}$ & 0 & & " & 20 & » & 0,3639 & 0,3639 \\
\hline 24 & $A$ & 16.0 & 16.0.1 & 90 & 0 & 85 & $233^{\circ}$ & 8.5 & 23. & 0 & 0 & 85 & 23. & 0 & 12,408 & 0 & 12,408 \\
\hline 25 & a & 80 & 801 & & $\triangleright$ & 80 & 50 & 80 & 50 & & $\gg$ & 80 & 50 & » & 6,2040 & > & $6,20: 10$ \\
\hline 26 & $j$ & 40 & 11.0 .2 & & • & 76 & 48 & 76 & 48 & & 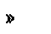 & 76 & 48 & * & 4,2652 & 3 & 4,2652 \\
\hline 27 & $B$ & 0 & 902 & & D & 74 & 0. & 74 & 0. & & 》 & 74 & $0^{\circ}$ & 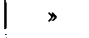 & 3,4897 & > & 3,4897 \\
\hline 28 & $v$ & 30 & 301 & & » & 64 & $44^{\circ}$ & 66 & $44^{\circ}$ & & » & 66 & $44^{\circ}$ & 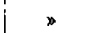 & $2,3 \geq 65$ & ๖ & 2,3265 \\
\hline 29 & $\boldsymbol{\varphi}$ & 20 & 201 & & 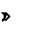 & 57 & $11^{\circ}$ & 57 & 11. & & $\infty$ & 37 & $11^{\circ}$ & $\nu$ & 1,5510 & $D$ & 1,5510 \\
\hline 30 & $x$ & 50 & 503 & & 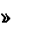 & 52 & $16^{\circ}$ & 52 & $16^{\circ}$ & & 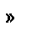 & 52 & $16^{\circ}$ & $\nabla$ & $1,292 \mathrm{i}$ & , & 1,2925 \\
\hline 31 & $o$ & 10 & 101 & & 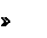 & 37 & 48 & 37 & 48 & & 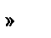 & 37 & 48 & B & 0,7755 & > & 0,7755 \\
\hline tg & 4 & $\frac{1}{2} 0$ & 102 & & $\gg$ & 21 & 11. & 21 & 11. & & " & 21 & 11. & $\star$ & 0,3877 & , & 0,3877 \\
\hline 38 & $\beta$ & $\frac{1}{3} 0$ & 103 & & > & 14 & 29 & 14 & 29. & & 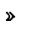 & 14 & 29. & $"$ & 0,2585 & - & 0,2585 \\
\hline
\end{tabular}


Ueber Anglesit von Monteponi (Sardinien).

\begin{tabular}{|c|c|c|c|c|c|c|c|c|c|c|c|c|c|c|c|c|c|c|}
\hline $\mathrm{Nr}$. & 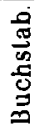 & $\begin{array}{l}\frac{\Phi}{0} \\
\frac{0}{E} \\
\dot{n}\end{array}$ & $\stackrel{\stackrel{D}{\Xi}}{\Xi}$ & & $p^{\prime \prime}$ & & $\varphi^{\prime \prime}$ & & $0^{\prime \prime}$ & & $0^{\prime \prime}$ & & $\xi "$ & & $\eta^{\prime \prime}$ & $\left(\begin{array}{c}x^{\prime \prime} \\
\text { (Prismen) } \\
\left(x^{\prime \prime}: y^{\prime \prime}\right)\end{array}\right.$ & $y^{\prime \prime}$ & $\begin{array}{l}d^{\prime \prime}= \\
\operatorname{tg} \varrho^{\prime \prime}\end{array}$ \\
\hline 34 & $k$ & 24.00 & 24.1 .0 & 860 & & 900 & $0 \quad 0^{\prime}$ & $90^{\circ}$ & $0^{\prime}$ & 900 & $0^{\prime}$ & $86^{\circ}$ & $005^{\prime}$ & & 0 & $14,613$. & $\infty$ & $\infty$ \\
\hline 35 & $\boldsymbol{E}$ & $22 . \infty$ & 22.1 .0 & 85 & 44 & & 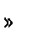 & & 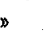 & . & 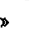 & 85 & 44 & & 16 & 13,396 & \$ & 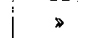 \\
\hline 36 & $Z$ & $16 . \infty$ & 16.1 .0 & 84 & & & $»$ & & » & & 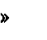 & 84 & 8 & & 52 & 9,7494 & $\gg$ & * \\
\hline 37 & $F^{r}$ & $15 . \infty$ & 15.1 .0 & 83 & 45 & & » & & » & & 》 & 83 & 45 & 6 & 45 & 9,1335 & $»$ & 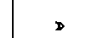 \\
\hline 38 & $G$ & 800 & 840 & 78 & 24 & & " & & D & & 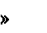 & 78 & 24 & 11 & 36 & 4,8712 & $\gg$ & \# \\
\hline 39 & $H$ & $\frac{15}{2} \infty$ & 152.0 & 77 & 39 & & » & & 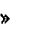 & & » & 77 & 39 & 12 & 21 & $4,5667$. & $»$ & $»$ \\
\hline 40 & $J$ & $7 \infty$ & 710 & 76 & 48 & & » & & * & & 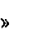 & 76 & 48 & 13 & 12 & 4,2623 & 》 & $»$ \\
\hline 41 & $K$ & $6 \infty$ & 610 & 74 & 41. & & 》 & & 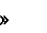 & & , & 74 & $41^{\circ}$ & 15 & 18 & 3,6534 & 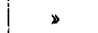 & $n$ \\
\hline 42 & $Y$ & $\frac{2}{2} \infty$ & $9 \geq 0$ & 69 & 57 & & $\triangleright$ & & 》 & & $\triangleright$ & 69 & 57 & 20 & 3 & $2,7400^{\circ}$ & $D$ & » \\
\hline 43 & $l$ & $4 \infty$ & 410 & 67 & 41 & & $D$ & & $\triangleright$ & & » & 67 & 41 & 22 & 19 & 2,4356 & $\triangleright$ & $»$ \\
\hline 44 & $e$ & $3 \infty 0$ & 310 & 61 & $18^{\circ}$ & & " & & $"$ & & $\gg$ & 61 & 18. & 28 & $41^{\circ}$ & 1,8267 & > & , \\
\hline 45 & $d$ & $2 \infty$ & 210 & 50 & $36^{\circ}$ & & » & & $\triangleright$ & & s & 50 & $36^{\circ}$ & 39 & 23. & 1,2178 & $\triangleright$ & » \\
\hline 46 & $\Theta$ & 61 & 611 & 74 & 41. & 78 & 17. & 77 & 32. & 51 & 51. & 70 & 49 & 14 & 59 & 30 & 1,2736 & 4,8219 \\
\hline 47 & $f$ & 41 & 411 & 67 & 41 & 73 & 23. & 72 & 8 & & " & 62 & 26 & 21 & $90^{\circ}$ & 3,1020 & $\$$ & 3,3527 \\
\hline 48 & $g$ & 31 & 311 & 61 & $18^{\circ}$ & 69 & $20^{\circ}$ & 66 & 44 & & $D$ & 55 & 10 & 26 & 42 & 2,3265 & $\triangleright$ & 2,6522 \\
\hline 49 & $r$ & 21 & 211 & 50 & $36^{\circ}$ & 63 & 31 & 57 & 11. & & $\gg$ & 43 & 46 & 36 & $36^{\circ}$ & 1,5510 & s & 2,0071 \\
\hline 50 & $\approx$ & 1 & 111 & 31 & $20^{\circ}$ & 5 6 & 9 & 37 & 48 & & $D$ & 25 & 35 & 45 & 11 & 0,7755 & 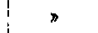 & 1,1910 \\
\hline 51 & $\boldsymbol{\tau}$ & 21 & 122 & 16 & 56 & 53 & $\mathbf{5}$ & 21 & $11^{\circ}$ & & D & 13 & 28 & 49 & 54 & $0,3877^{\circ}$ & $>$ & 1,3310 \\
\hline 52 & $\underline{\xi}$ & $\frac{1}{3} 1$ & 133 & 11 & 28. & 32 & 25. & 14 & 29. & & 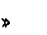 & 9 & 4 & 60 & $57^{\circ}$ & 0,2585 & 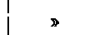 & 1,2997 \\
\hline 53 & $\nu$ & $z$ & 221 & 31 & $20^{\circ}$ & 71 & 28 & 57 & $11^{\circ}$ & 68 & 34 & 29 & 33 & 54 & $4^{\circ}$ & 1,5510 & 2,5472 & 2,9829 \\
\hline 54 & $t$ & $\frac{1}{2}$ & 112 & & $»$ & 36 & 42. & 21 & $11^{\circ}$ & 32 & $29^{\circ}$ & 18 & 6 & 30 & 42 & $0,3877^{\circ}$ & 0,6368 & 0,7456 \\
\hline 55 & $E$ & $\frac{1}{3}$ & 113 & 31 & $20^{\circ}$ & 26 & 25. & 14 & $29^{\circ}$ & $2: 3$ & 0 & 13 & 23 & 22 & $20^{\circ}$ & 0,2585 & $0,4 \geq 45$ & 0,4369 \\
\hline 56 & $I$ & $1 \frac{1}{4}$ & 12.1 .12 & 282 & 12. & ? 38 & 3 & 37 & 48 & 6 & 3. & 37 & 38 . & 4 & $47^{\circ}$ & 0,7755 & 0,1061 & 0,7827 \\
\hline 57 & $q$ & $1 \frac{2}{6}$ & 616 & 74 & 41. & 38 & 48 & & » & 11 & 59 & 37 & 11 & 9 & 31. & " & 0,2122 & $\cdot 0,8040$ \\
\hline 50 8 & $\pi$ & $1 \frac{1}{5}$ & 315 & 71 & 49 & 39 & 13. & & s & 11 & $17^{\circ}$ & 36 & 55 & 11 & 23 & \# & 0,2547 & 0,8163 \\
\hline 59 & $\%$ & 11 & 414 & 67 & 41 & $\$ 9$ & 38. & & : & 17 & 40 & 36 & 28 & 14 & 7 & » & 0,3484 & 0,8383 \\
\hline 60 & $\psi$ & $1 \frac{1}{3}$ & 313 & 61 & 18. & 41 & 29 & & 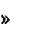 & 23 & 0 & 35 & 31 & 18 & 32. & 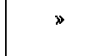 & 0,4245 & $0,85: 12$ \\
\hline 61 & $D$ & $1 \frac{9}{5}$ & 325 & 36 & 42 & 42 & 51. & & $\gg$ & 26 & $59^{\circ}$ & 34 & 39 & 21 & 55 & " & 0,5094 & $0, ? 9279$ \\
\hline $6 z$ & $y$ & $1 \frac{1}{2}$ & 212 & 亏ั0 & $36^{\circ}$ & 45 & 6 & & 》 & 32 & $29^{\circ}$ & 33 & $11^{\circ}$ & 26 & 43 & » & 0,6368 & 1,0035 \\
\hline 63 & $\iota$ & $1 \frac{2}{3}$ & 323 & 48 & $24^{\circ}$ & 48 & $59^{\circ}$ & & » & 40 & 20 & 30 & $35^{\circ}$ & 33 & 51. & $\triangleright$ & $0,8490^{\circ}$ & $\cdot 1,1500^{\circ}$ \\
\hline 64 & $\omega$ & 42 & 421 & 50 & $36^{\circ}$ & 76 & 0 & 72 & 8 & 68 & 34 & 48 & 35 & 38 & 0 . & 3,1020 & 2,5472 & 4,0108 \\
\hline 65 & $s$ & 34 & 213 & ! & $\gg$ & 33 & 47 & 27 & 20. & 23 & 0 & 25 & $2:$ & 20 & 40 & 0,5170 & 0,4245 & 0,6690 \\
\hline 66 & $\zeta$ & 4 & 214 & & 》 & 26 & 38. & 21 & 11. & 17 & 39. & 20 & $16^{\circ}$ & 16 & 32 & 0,3877 & 0,3184 & $0,5016^{\circ}$ \\
\hline 67 & $L$ & $2 \frac{2}{3}$ & 623 & 61 & 18 & 60 & 30. & 57 & 11. & 40 & 20 & 49 & $46^{\circ}$ & 24 & 42. & 10 & 490 & 1,7681 \\
\hline 68 & $\gamma$ & 3 & 312 & & $"$ & 52 & 59 & 49 & 19 & 32 & $29^{\circ}$ & 44 & 27. & 22 & 32 & 1,1632 & 0,6368 & 1,3262 \\
\hline 69 & $\mathfrak{a}$ & $\frac{3}{4} \div$ & 314 & & 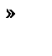 & 33 & 33 & 30 & 11 & 17 & 39 & 29 & 0 & 15 & 23. & 0,5816 & 0,3154 & 0,6631 \\
\hline 70 & $\Im$ & $2 \frac{1}{10}$ & 20.1 .10 & 85 & $18^{\circ}$ & 57 & $16^{\circ}$ & 57 & 11. & 7 & 15. & 56 & $58^{\circ}$ & 3 & 57 & 1,5510 & 0,1273 & 1,4975 \\
\hline 71 & $\mu$ & $2:$ & 412 & 67 & 41 & 39 & 11 & & 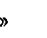 & 32 & 29. & 52 & $36^{\circ}$ & 19 & 2 & $\gg$ & 0,6368 & 1,6764 \\
\hline $7 i$ & $p$ & $2^{3}$ & 432 & 39 & $4^{\circ}$ & 67 & $\mathbf{5 3}$ & & 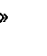 & 62 & 22 & 35 & 44 & 45 & 59 & $>$ & $i^{1,9104}$ & 2,3426 \\
\hline 73 & $\varrho$ & 17 & 234 & ¡22 & 5 . & 40 & 52. & 21 & 11. & 43 & 41 & 15 & 40 & 41 & 41 & $0,3877^{\circ}$ & 0,9532 & 1,0316 \\
\hline 74 & $\eta$ & $\frac{5}{2}$ & 512 & 71 & 49 & 63 & 53. & 62 & 43 & 32 & $29^{\circ}$ & 58 & $39^{\circ}$ & 16 & $16^{\circ}$ & $1.9387^{\circ}$ & $0,636 \mathrm{~s}$ & 2,0405 \\
\hline 75 & $\dot{\Gamma}$ & $\frac{5}{3}-\frac{1}{3}$ & 513 & & s & 53 & 41 & 52 & $16^{\circ}$ & 23 & 0 & 49 & 57 & 14 & 34 & 1,292; & 0,4245 & 1,3605 \\
\hline
\end{tabular}


P. Hermann. Ueber Anglesil von Monteponi (Sardinien).

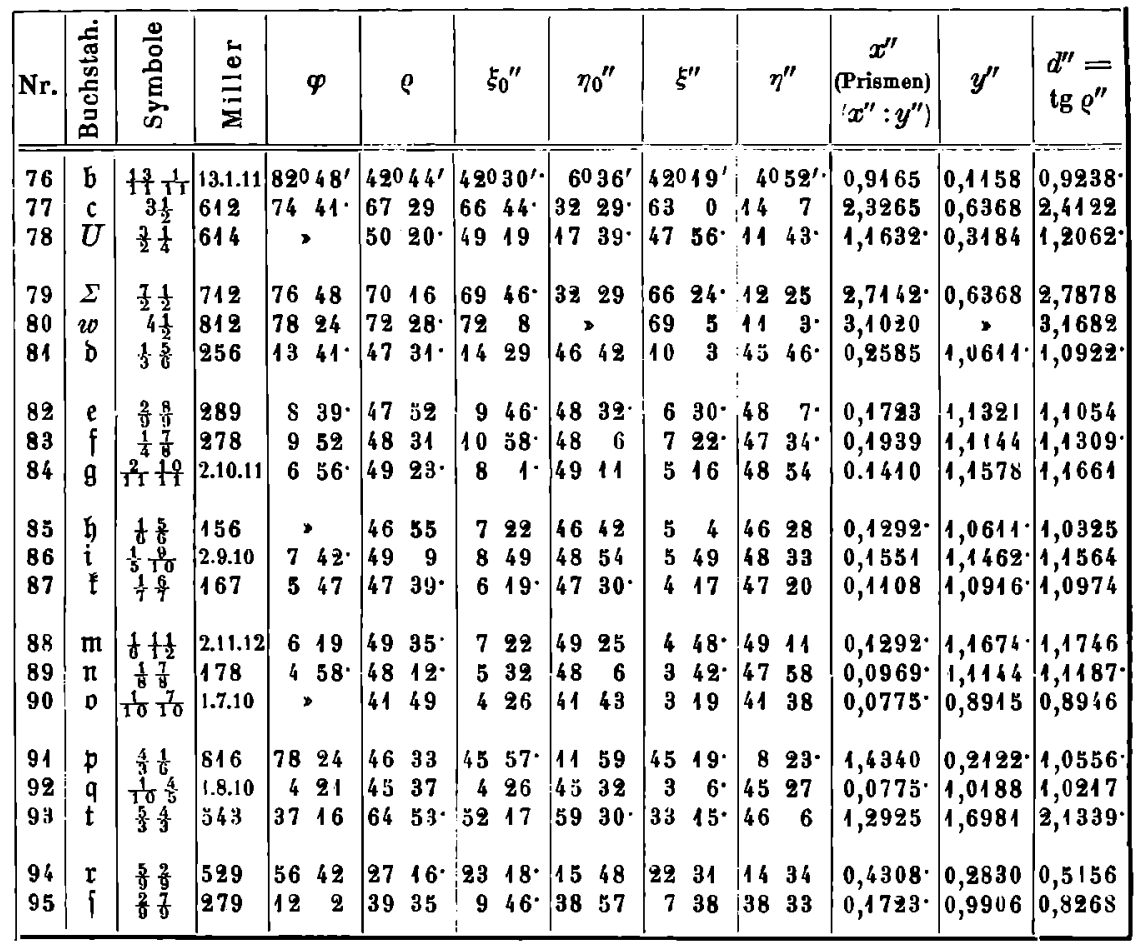

Vorliegende Arbeit wurde im krystallographischen Institute zu Heidelberg angefertigt und die Untersuchungen dazu daselbst durchgeführt. Es sei mir an dieser Stelle gestattet, meinem verehrten Lehrer, Herrn Prof. Goldschmidt in Heidelberg, den aufrichtigsten Dank auszusprechen für die Unterstützung und Förderung dieser Arbeit durch seine Rathschläge und Anleitungen, die er mir stets bereitwilligst ertheilte.

Ileidelberg, den 3. Februar 1904.

Paul Hermann. 